\title{
Introduction to Complex Numbers
}

by Mashudu Mokhithi \& Jonathan Shock

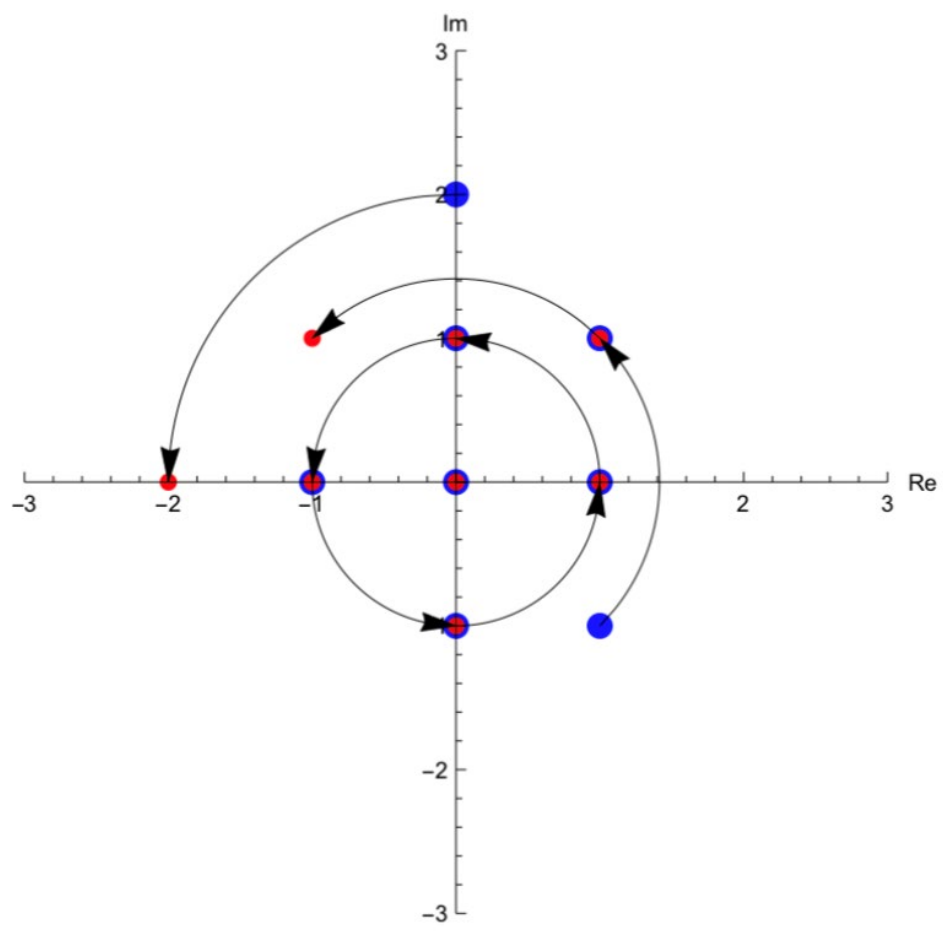

Department of Mathematics and Applied Mathematics

University of Cape Town 
Version 1, April 2020

(C) Mashudu Mokhithi and Jonathan Shock. Licensed under a Creative Commons Attribution 4.0 International licence (http://creativecommons.org.licenses/by/4.0/).

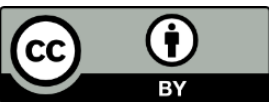

\section{Acknowledgements}

With many thanks to Professor John Webb and Dr Jurie Conradie whose notes were a foundation and motivation for much of what is here.

\section{Author contact details}

Mashudu Mokhiti<mashudu.mokhiti@uct.ac.za>

Jonathan Shock<jonathan.shock@uct.ac.za>

This work forms part of the MAM1000 chapter series and is part of the forthcoming Introduction to University Level Mathematics for a South African Audience open textbook. It has been written with a focus on first-year students at the University of Cape Town, South Africa, but can be applied in any mathematics education context. 


\section{Contents}

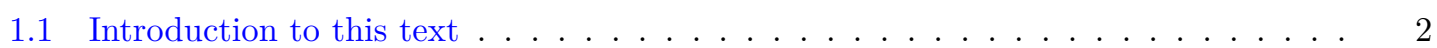

1.2 Number Systems and a new way to look at mathematics . . . . . . . . . . 3

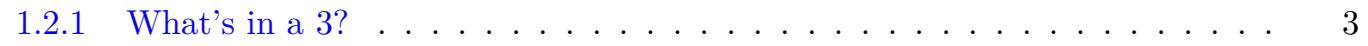

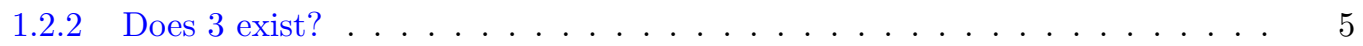

1.2.3 On the freedom we have in Mathemafrica . . . . . . . . . . . 6

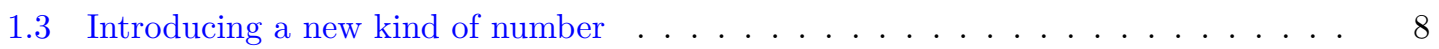

1.4 Another perspective on addition and multiplication . . . . . . . . . . 9

1.5 The Complex Plane . . . . . . . . . . . . . . . . . . . . . . . 13

1.6 Simple arithmetic operations on complex numbers . . . . . . . . . . . . 17

1.7 Identities, inverses and division of complex numbers . . . . . . . . . . . . 26

1.8 Properties of the conjugate and modulus . . . . . . . . . . . . . . . 32

1.9 The argument of a complex number . . . . . . . . . . . . . . . 37

1.10 The Modulus-Argument form of a complex number . . . . . . . . . . . . . . 41

1.11 Multiplication and de Moivre's Theorem . . . . . . . . . . . . . . . . . . . . 44

1.12 Taking powers of complex numbers . . . . . . . . . . . . . . . . 47

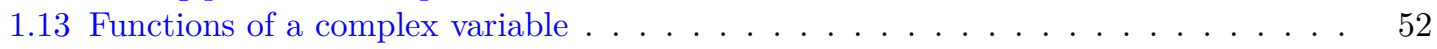

1.14 The Complex Exponential Function _ . . . . . . . . . . . . . . . 53

1.15 Complex trigonometric functions . . . . . . . . . . . . . . . . . . 64

1.16 Complex $n^{t h}$ roots of a complex number . . . . . . . . . . . . . 68

1.17 Zeros of polynomials in a complex variable . . . . . . . . . . . . 73 


\section{Chapter 1}

\subsection{Introduction to this text}

These notes are meant as an introductory exploration of complex numbers. They are structured to be both readable and thorough such that going through them, and doing all of the exercises should put you in a good place for understanding the foundation of this fascinating subject. There is a great deal more that can, and has, been said about complex numbers, so consider this your first journey into this world.

The real work on complex numbers starts with chapter 3 , so chapter 2 is just getting you thinking about number systems in general and indeed what a number really means. This is a little historical and philosophical aside, but I hope that it will get you thinking about some things which you probably thought you knew before, but maybe hadn't considered all that carefully.

Finally, these notes are a work in progress. There are likely many typos in them, so if you find any, please email jon.shock@gmail.com and we will endeavour to fix it as soon as we can.

Most of all, enjoy! 


\subsection{Number Systems and a new way to look at mathematics}

\subsubsection{What's in a 3?}

What is a 3 ? This might seem like a completely ridiculous question, but there are several answers to this:

- It is a particular pattern of lines, which are usually seen on a piece of paper, on a computer screen or on a blackboard.

- It is a symbol denoting a sound, though that sound is different in different languages (three, zintathu, sami, and so much more....)

- It is a symbol which denotes a concept.

- and probably more answers which I haven't thought of here...can you think of more?

Even the third answer has surprisingly different interpretations:

- It denotes the size of a collection of objects where the number of objects is denoted by the symbol 3 .

- It is a mathematical object which can be manipulated in an abstract space...wait, what?

It is quite remarkable that we can consider the number 3 without actually discussing any objects. When I say '3', you don't say: '3 what?'. You understand that this is a number which doesn't have to be associated with an object. In fact, you know that I can use it to manipulate other numbers: I can perform the "multiply by 3 " operation on a number to get a different number (unless the original number was 0). I can think of it as the number of times that I multiply another symbol by itself...things get even more abstract when that symbol is $x$, but we don't complain about $x^{3}$ and say "but what is $x "$ "? At least I hope not.

Ok, so we can agree that the symbol 3 has particular mathematical significance. I think that we can also agree that if we used the Chinese symbol 三, pronounced "san" which, yep, means 3 in Chinese, we'd all be talking about the same concept as ' 3 '

it's rather nice that in Chinese, the numbers 1, 2 and 3 are 一, 二, 三, though 4 and onwards are not so clearly linked to their numerical value. See Akkadian for a language which takes this even further.

So, as long as we agree on the symbol, it doesn't matter much what it looks like (though the simpler the better in general).

However, there are different ways that we can start to build up more complicated numbers. It's clear that the numbers we use would be very complicated if for every number we needed to introduce a completely new symbol. So, we start building up bigger numbers from smaller ones.

The number system most of us use now is called the Hindu-Arabic number system and was developed in India around 1500 years ago. They were taken from India through the Middle East and then through North Africa, before Fibonacci (who didn't invent the Fibonacci sequence!), introduced them to Europe.

Before this time, Europeans used the Roman Numeral system, which is a crazy system! The Hindu-Arabic number system is base 10: we build up larger (and smaller) numbers, in powers of 10. The Roman Numeral system is base 10...and (sub)base 5...and (sub)base 2...and depending on which order the numbers are in, a number is added or subtracted.

With $I=1, V=5$ and $X=10$, one to ten in Roman numerals is:

$$
I, I I, I I I, I V, V, V I, V I I, V I I I, I X, X
$$

So 8 has 4 digits and 9 has 2 digits, which is completely ridiculous, frankly! 
In fact, it's so crazy that even addition and multiplication using Roman numerals is really hard. This actually held back the development of European mathematics enormously. It was only because of the introduction of the Hindu-Arabic numeral system from North Africa that mathematics began to flourish in the 12th century, and allowed for the development of algebra in particular (named after al-Khwarizmi, a Persian scholar of the 9th century).

Although Chinese has a different set of symbols for the first ten numbers, it still uses the decimal system (base ten). In fact, the majority of languages use the decimal system, although not all of them do. Take a look here to see which languages use other bases. Some examples of base 10 number systems are can be found here. We should note that really the only good reason for using base 10 is because we have 10 fingers. The Mayans who noted that they also had 10 toes, decided to use a base 20 system:

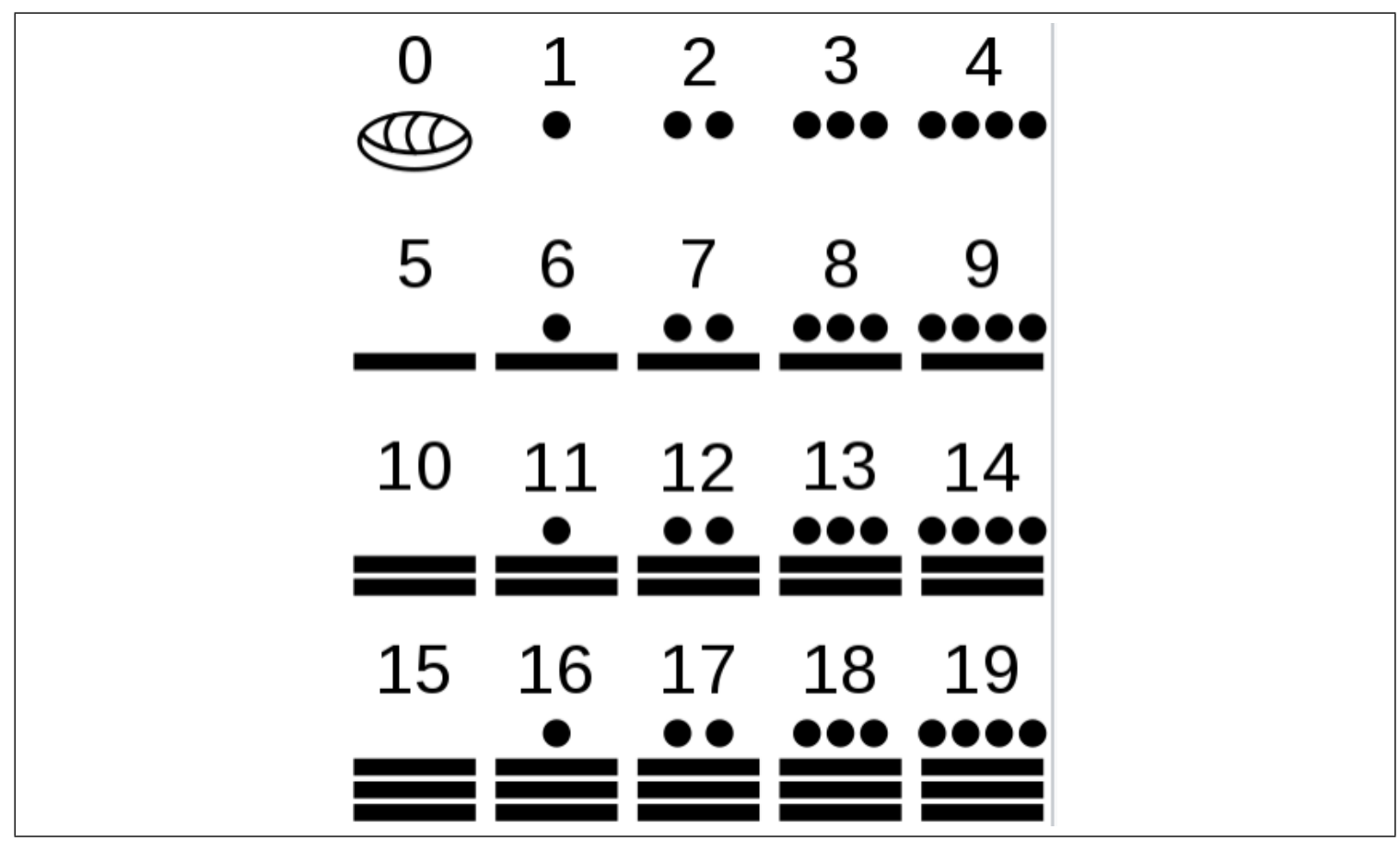

Figure 1.1: The base 20 Mayan number system

It turns out that in fact if we had 8 or 16 fingers and toes, we might be more natural mathematicians, as powers of 2 are generally much easier to work with.

Some languages went even higher and the Mali Empire, which at its peak (around the 14th century) was a civilisation with over 400 cities, towns and villages used base 60 (though there seems to be little reference on this). 
One of the most interesting and complex number systems in use today is that of Yoruba which uses a base 20 number system as well as using a system of subtraction, like the Roman numerals. You can see the way numbers are composed in Yoruba as taken from here:

$$
\begin{aligned}
35(\text { marundilogoji }) & =(2 \times 20)-5 \\
47 \text { (metadiladota }) & =(3 \times 20)-10-3 \\
51(\text { mokanleladota }) & =(3 \times 20)-10+1 \\
55(\text { marundilogota }) & =(3 \times 20)-5 \\
67(\text { metadiladorin }) & =(4 \times 20)-10-3 \\
73(\text { metaleladorin }) & =(4 \times 20)-10+3 \\
86(\text { merindiladorun }) & =(5 \times 20)-10-4 \\
117 & =(6 x 20)-3
\end{aligned}
$$

See if you can figure out the structure from the number words and the way they are composed. More numbers can be found here.

In fact, we can go back even further than the Roman and Hindu-Arabic number system to discover something about the symbolic expression of number.

The very first object we have today which has what appear to be numerical markings is the Ishango bone, found in the Kingdom of eSwatini (formerly Swaziland) and dates back 35,000 years. A good writeup of this, and the cradle of mathematics can be found on Mathemafrica.

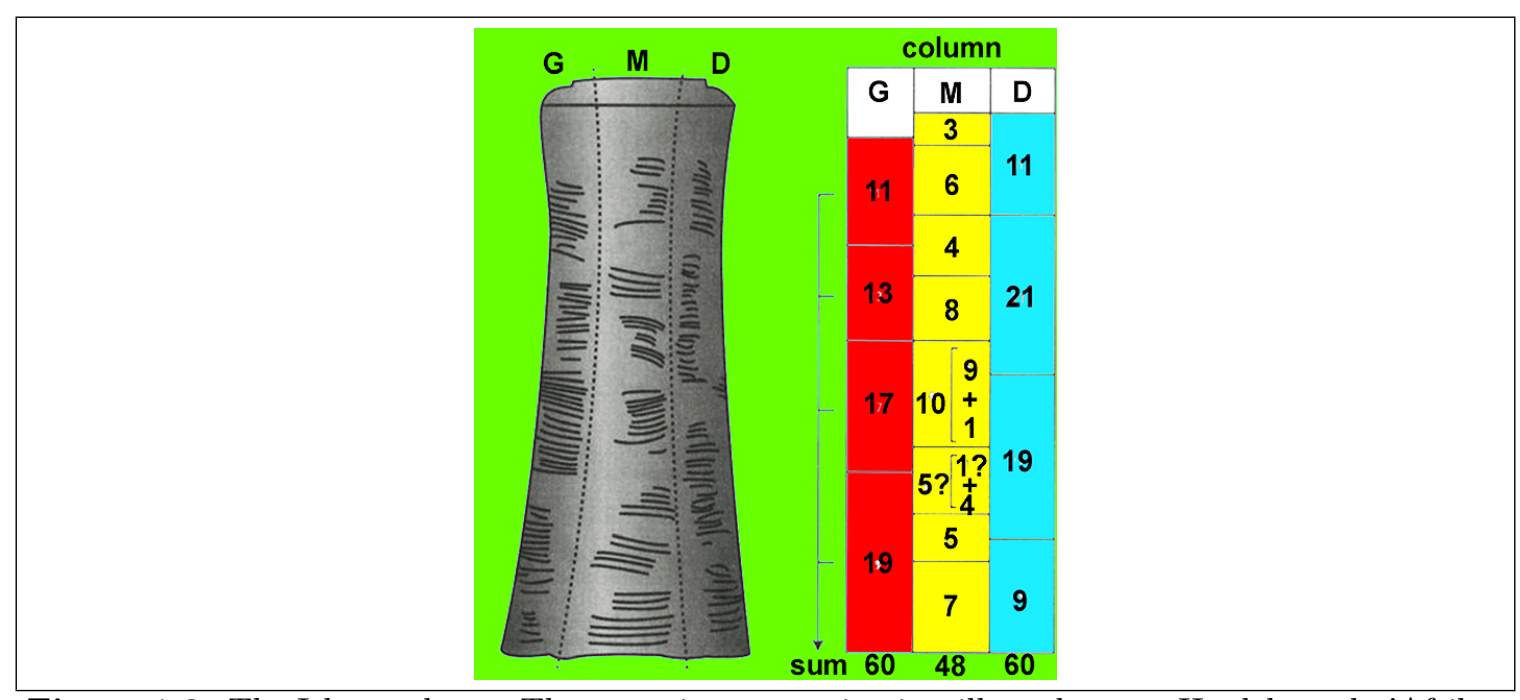

Figure 1.2: The Ishango bone. The exact interpretation is still not known. Huylebrouck, 'Afrika, die Wiege der Mathematik', Ethnomathematik, Spektrum der Wissenschaft 2 (2006), 10-15.

If you speak a language which has a number system different to any of those above, let me know and I'll be happy to include some information about it in a future edition.

\subsubsection{Does 3 exist?}

Have you ever seen a 3 in the wild? I mean, really seen it...? Yes, you've all seen the symbol, and you've seen collections of 3 things, but we can all agree that 3 has a meaning in an abstract way, without it being linked to any objects. Have you seen a -3 ? That's maybe even harder to see what it corresponds to as an entity (though we understand completely what it means as a mathematical object). How about $\pi$ ? Or $e$ ? Each of these has a very precise meaning, independent of the physical world, though they can be associated with real things in the real world.

The word "Table Mountain" is also just lines on a page, but it also corresponds to something that we can see and touch. Numbers are different. Numbers can have parallels in the real world, 
but really they live in an abstract space. Mathematics is (amongst other things) about how to understand the relationships and interplay of these objects in this abstract world, and about how to write the dictionary between the abstract world of numbers, and the real world. Let's call this abstract world Mathemafrica.

This has some very profound consequences. It means that so long as we are consistent, we have a playground to experiment in (or perhaps a laboratory to play in). We can start to make up the rules and see what the consequences are. We define the + symbol to have a particular action in Mathemafrica and then see what happens when we apply it to certain objects which live in this land.

We are now the inventors and discovers of this landscape. We get to define a rule, and then discover what it means...if we find that the result of this new rule are that it clashes with our former rules, then these rules are not compatible and we have to be careful when we apply them. I could try and define a rule which says that when I am counting, when I get to 9 and add 1 , I get back to 0. This, it turns out is a subregion in Mathemafrica called Modulandia, and it has very special rules of its own.

This newfound freedom is wonderful, but we have to be careful...so far, the beings in our land are the rational numbers, abstract symbols (like $x$ and $y$ and $n$ which we can, if we want associate with numbers, but we don't have to), functions, which are like the transport infrastructure in Mathemafrica, and the society in this land is made up of all the possible interactions of these objects.

What we are going to see next is that actually, if we want a more flexible society, we can add some new people to Mathemafrica and they will allow us to do things which we were previously completely impossible. We will see this in the next section...

\subsubsection{On the freedom we have in Mathemafrica}

So, how much do we discover about Mathemafrica, and how much do we invent ourselves? Well, to a certain extent, we get to define a set of our own objects, and then we see how they interact in the mathematical world. One of the best examples of this is the square root function. This is something that so often trips up first years. A question I often get is:

I thought that the square root could be positive or negative. Isn't $\sqrt{9}= \pm 3$ ?

The answer is that we get to define the square root function as we want. It isn't a naturally occurring function which you find in the wild and one that has properties of its own. We invent this function whose definition we choose to be:

Definition 1.2.1. The principle square root of $x$ (written $\sqrt{x}$ )

The principle square root of a positive real number $x$ is that positive number which, when squared, gives you $x$.

Notice here that we define the principle square root from the start as being the positive number. And that is our choice. We could have chosen the negative, but having chosen the positive, we can then explore how this object can be used, and what its consequences are. Note that generally we simply say square root for principle square root and implicitly mean the positive root. This is an issue of language. Indeed there are two square roots of 9, but when we say "the square root of 9", we mean the principle, or positive square root.

Note that $x^{2}=9$ has two solutions, one is $\sqrt{9}=3$ and one is $-\sqrt{9}=-3$, but $\sqrt{9}$ itself is positive. To get the negative root, we have to put the - sign on the front.

In the following we will frequently make definitions of mathematical functions, and then explore their consequences as we add them as another ingredient to Mathemafrica.

The bottom line: 
1. We can define new mathematical operations, so long as they aren't in contradiction to the rules of the game that came before (except that sometimes they will stretch the previous rules).

2. We can use these definitions and the others that we have made before in order to prove theorems.

We will be careful in what follows to distinguish here what is a definition and what is a theorem and show you how to prove the theorems from the definitions. 


\subsection{Introducing a new kind of number}

What if you knew nothing about negative numbers and I asked you to solve the equation

$$
x^{2}=1
$$

You would laugh at how ridiculous I was being and tell me the answer was 1 not realising you'd missed half of the solutions.

In China, around 200BC negative numbers were first introduced. This might be a long time ago, but ideas of counting had been around for a long long time already and so we had mathematics for millenia without having negative numbers.

In Indian mathematics negative numbers were introduced around 620AD with Brahmagupta who introduced them in the concept of debt.

In Greek mathematics where many of the cornerstones of modern mathematics developed, they didn't really concern themselves with negative numbers because they were mostly interested in geometry, and when you're thinking about distances, negative numbers aren't really needed.

In 300AD Diophantus wrote down an equation which would have had a negative solution and said that this was absurd!

Really, negative numbers were understood quite a bit later with the development of mathematics in the Arab world, with the likes of Al-Khwarizmi, Abul-Wafa and Al-Samawal.

If you want to know more, take a look at https://nrich.maths.org/5961.

What if you didn't believe in the existence of irrational numbers and I asked you to find $x$ such that

$$
x^{2}=2
$$

You would laugh at how ridiculous I was being and tell me that there is no solution to this. You would be in good company! See The wikipedia article on Irrational Numbers for the history of them.

We have a good way of denoting negative numbers. We just take the magnitude of the number and put a little horizontal line in front of it. It's just some notation, but we know what it means and we know how to manipulate it. For irrational numbers we can't ever write down their digit expansions, but we can write things like $\sqrt{2}, e, \pi$ and we know what that means.

Well, we are now going to do exactly the same thing but this time with the equation:

$$
x^{2}=-1
$$

You first laugh and tell me how ridiculous I'm being as you have done so many times before. However, this time I take my hat off and pull out a new number...

The answer, I tell you, is $i$ and $-i$ !

What's that? You say. 
Well, they are both numbers which, when squared give you -1. That's just how we are going to define them. It need be no more, nor less complicated than that.

Definition 1.3.1. The imaginary unit $i$

$i$ and $-i$ are the two numbers which when squared give -1 .

$$
i^{2}=-1,(-i)^{2}=-1
$$

This is the starting point of complex numbers and as we will see, it will allow us to do many things that we couldn't do before, including solving equations, like that above, which we thought were unsolvable. It will also bridge areas of mathematics that we thought had nothing to do with each other before, like trigonometry and exponential functions. We'll discover that they are really the same thing, but that only becomes clear when we are allowed to define this weird number $i$, such that $i^{2}=-1$.

Looking at the equation $x^{2}=1$ and asking how to solve this using a graph means taking the graph of $y=x^{2}-1$ and asking when $y=0$. It's obviously where it cuts the $x$-axis. Asking to solve $x^{2}=-1$ we can look at the graph of $y=x^{2}+1$ and ask where this cuts the $x$-axis. And suddenly it is clear where the problem lies...it doesn't cut the $x$-axis. But then perhaps the $x$-axis isn't all there is. We will see this in the next section.

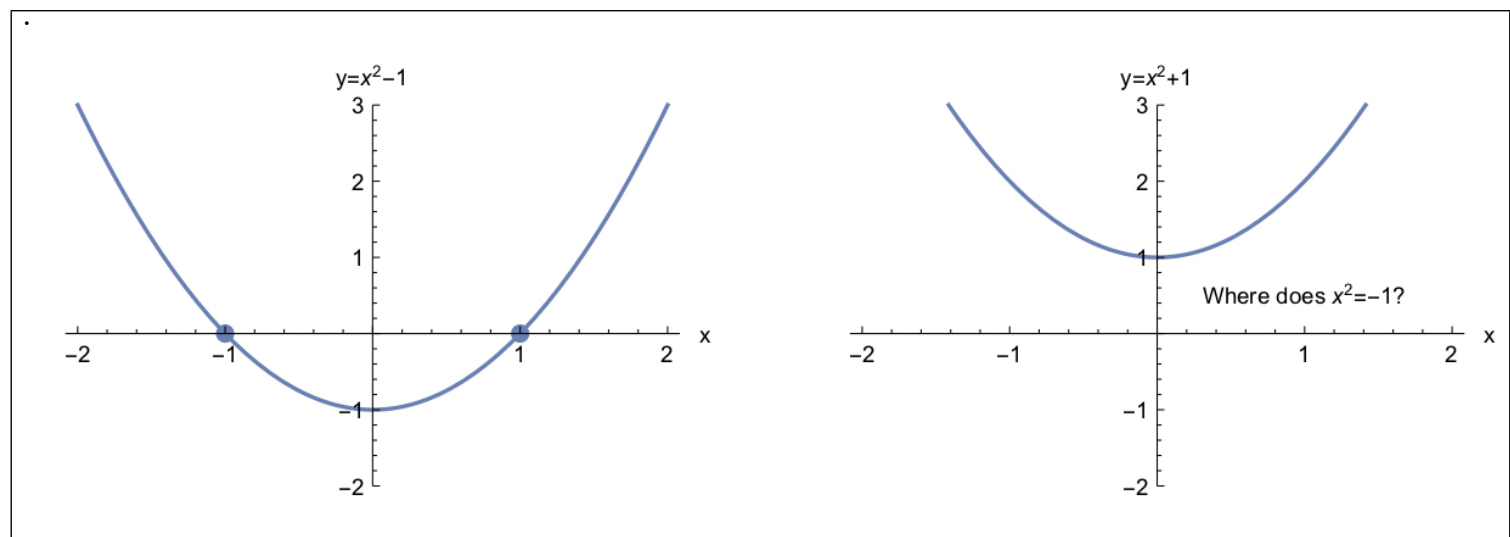

Figure 1.3: Solving $x^{2}=1$ is equivalent to asking where the graph of $y=x^{2}-1$ cuts the $x$-axis, but it's clear that we have a problem when we try and solve $x^{2}=-1$.

\subsection{Another perspective on addition and multiplication}

Let's look at this object in a slightly different way. The more ways we can look at the same thing, the clearer it will all be in the end.

So far we have been working with numbers on a number line (one dimension). There was an origin (0), and there were positive and negative numbers to the right and left of the origin and up to now every number we could imagine was on this line.

In particular we could move about this number line using the basic operations of arithmetic. Adding shifts you left or right. Multiplication by a positive number scales you away from the origin. Multiplication by a negative number reflects you about the origin and then scales you.

If we start at 1 and multiply by 2 we end up moving to 2 , obviously. 


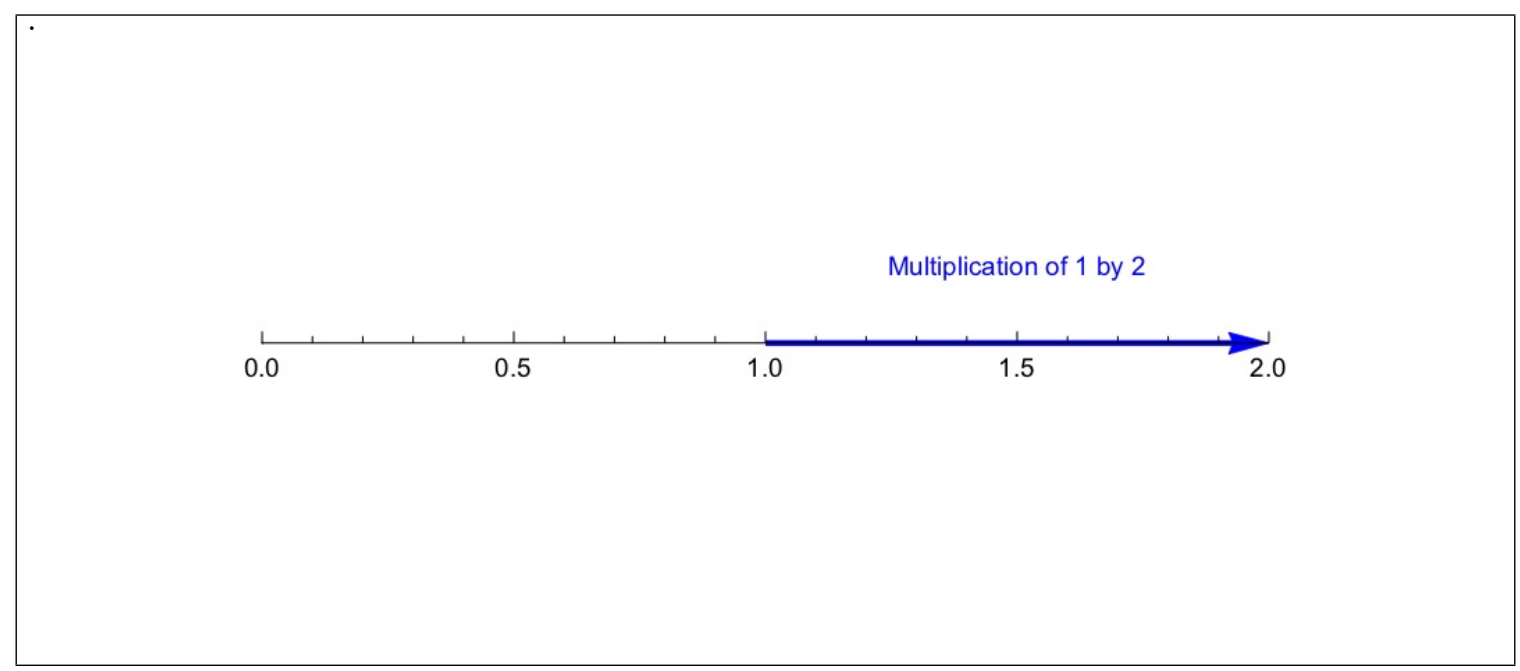

Figure 1.4: Multiplication by a positive number on the real number line is a scaling.

We can also ask about the square root of 2 . What that really means is: What is the operation (in terms of moving on the number line) which, when you do it twice takes you from 1 to 2 . Well, we can first multiply by $\sqrt{2}$ which takes us to $\sqrt{2}$ or indeed we can multiply by $-\sqrt{2}$ (reflect to -1 , then scale to $-\sqrt{2}$ ) and then multiply again by $-\sqrt{2}$ (reflect by -1 which takes us to $\sqrt{2}$ then scale by $\sqrt{2}$ which takes us to 2 ).

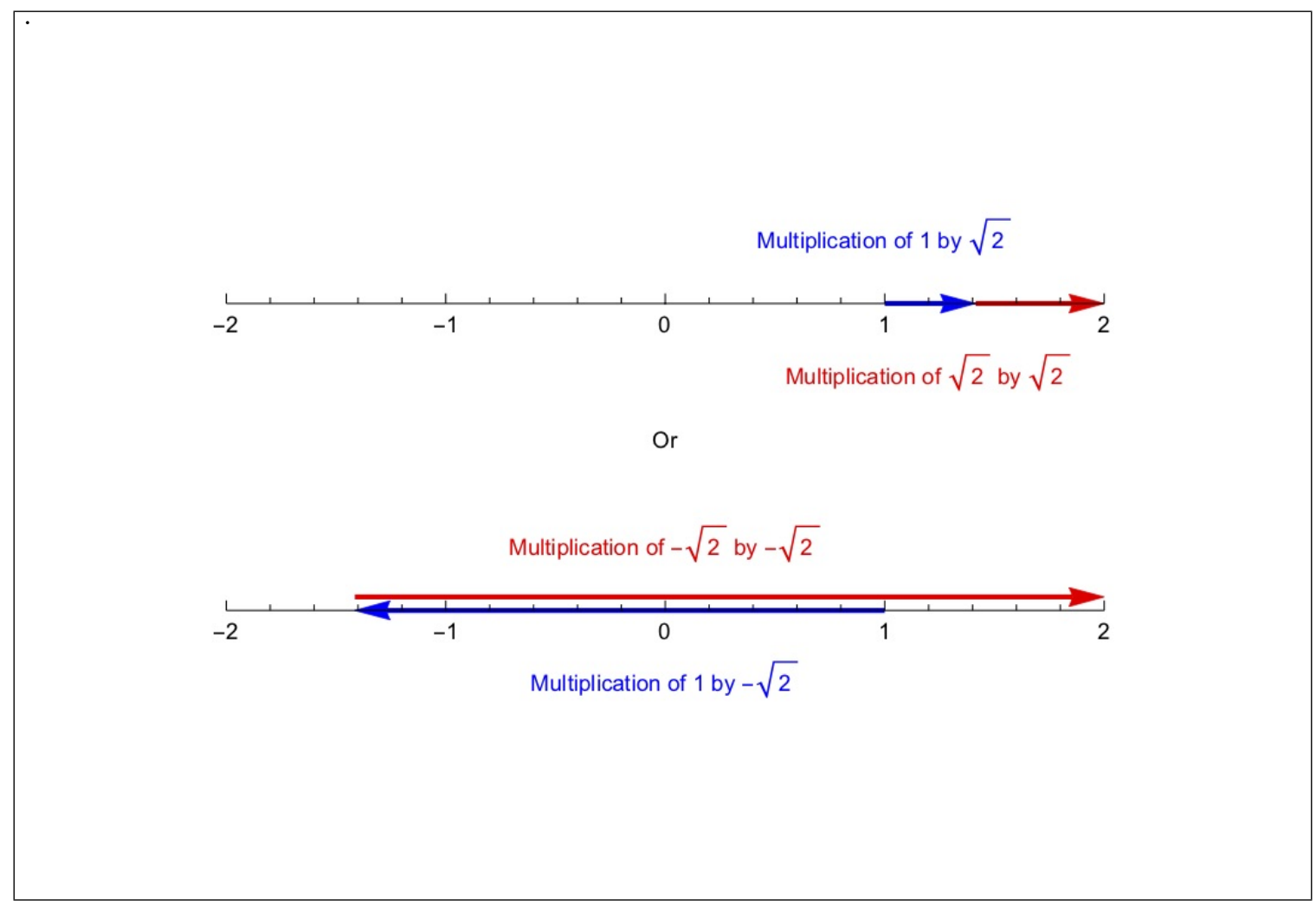

Figure 1.5: Multiplication by a positive number on the real number line is a scaling.

We could ask something a bit more tricky. We could ask if you can multiply by -1 in two equal steps. We saw above that reflecting twice (ie. multiplying by a negative number twice) keeps us on the same side of the origin. So how can we do something twice which takes us from 1 to -1 . Remember that we are talking about multiplication, not addition. It's easy to get from 1 to -1 in two steps by addition.

In fact, with the number line as it is we can't get from 1 to -1 multiplicatively in two equal steps. We are now going to upgrade our number line and turn it into a number plane and then all will become clear. 
Let's simply extend the number line into a number plane. The horizontal axis of this plane is just like the real numbers you know and love, and now there is a new vertical axis which so far doesn't mean much to us.

The wonderful thing about a plane is that we can move around in it in much more complex ways than we can on a line. On a line you can move left and right (either by addition or multiplication), and you can reflect about the origin (if you are multiplying by a negative number).

In a plane we can now rotate. Once we allow for rotation, it's very obvious how we can get from 1 to -1 in two steps...though it's not completely clear yet why this corresponds to multiplication...we will see!

Well, let's just start off by drawing a two dimensional space of numbers. We are going to call one of the axes "Re" (the real number line) and the other axis we will call "Im" (the imaginary number line). The reason for this will become clear soon I hope. Let's start off just by seeing what it would look like to rotate from 1 to somewhere on the imaginary number line and then rotate again to get to -1 .

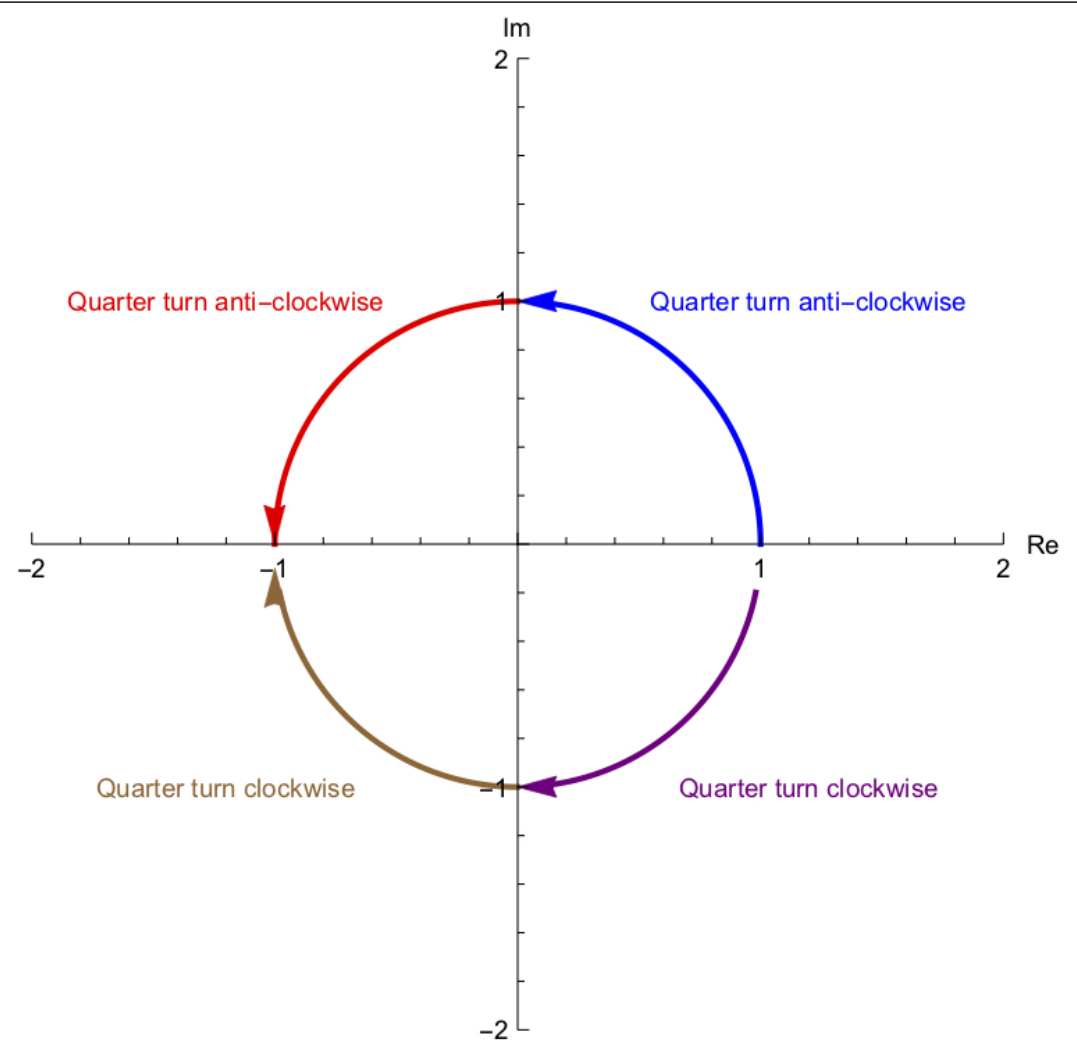

Figure 1.6: Rotation by $\frac{\pi}{2}$ or $\frac{-\pi}{2}$ twice to get us from 1 to -1 in two equal steps.

Note that just as with moving from 1 to 2 in two equal steps there are two ways to do this operation..

So...so far in this subsection we haven't mentioned this weird number $i$. However, we've actually just sneaked it in there without you really noticing it.

When we asked the question how do you get from 1 to 2 in two equal multiplicative steps we are really asking you to solve:

$$
1 \times x \times x=2
$$

for $x$. We could also just write this as $x^{2}=2$. The answer to this of course is $x= \pm \sqrt{2}$.

We could write that number at the point that we get to at the end of the first step and it would look like: 


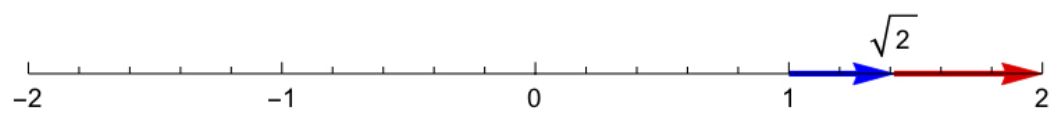

Or

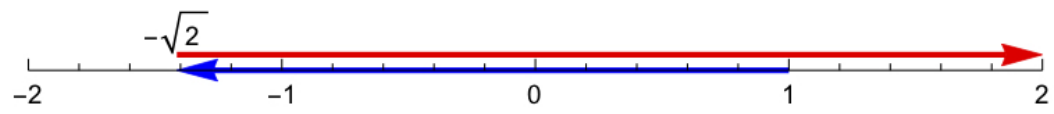

Figure 1.7: $\sqrt{2}$ and $-\sqrt{2}$ are the points that we get to after one step of the two to move us multiplicatively from 1 to 2 in two equal steps.

Now we are asking to solve:

$$
1 \times x \times x=-1
$$

or $x^{2}=-1$. And we know the answer to that now is $x= \pm i$.

So perhaps the points that we get to at the end of the quarter turns anti-clockwise and clockwise are $i$ and $-i$. In fact this is exactly how we are going to construct what is known as the complex plane.

When we introduced the imaginary number before, it was clear that it wasn't a number like we'd ever seen before, and didn't fit nicely on the real number line, so we are going to give it its own line and extend the number line to a number plane, with one axis being real numbers and the other axis being imaginary numbers.

That then looks like there are more numbers that are imaginary than just $i$. Well, yes, absolutely. To start with we could have, for instance, two lots of i, which we will write:

\section{$2 i$}

This is a number which, when you square it gives you:

$$
(2 i)^{2}=2^{2} i^{2}=4^{\prime} \times-1=-4
$$

In fact, we can take $i$ and we can multiply it by any real number, $a$ and then you just have $a$ lots of $i$, and this number lies somewhere on the imaginary number line.

You might think as just as you have 1,2,3,4, etc. along the real number line you might have $i, j, k, l$, etc. along the imaginary number line, but remember 2 is just $1+1$ and 3 is $1+1+1$, so $2 i=i+i$ and there isn't another way of writing this number. Along the imaginary direction we only ever have a real number times $i$ 


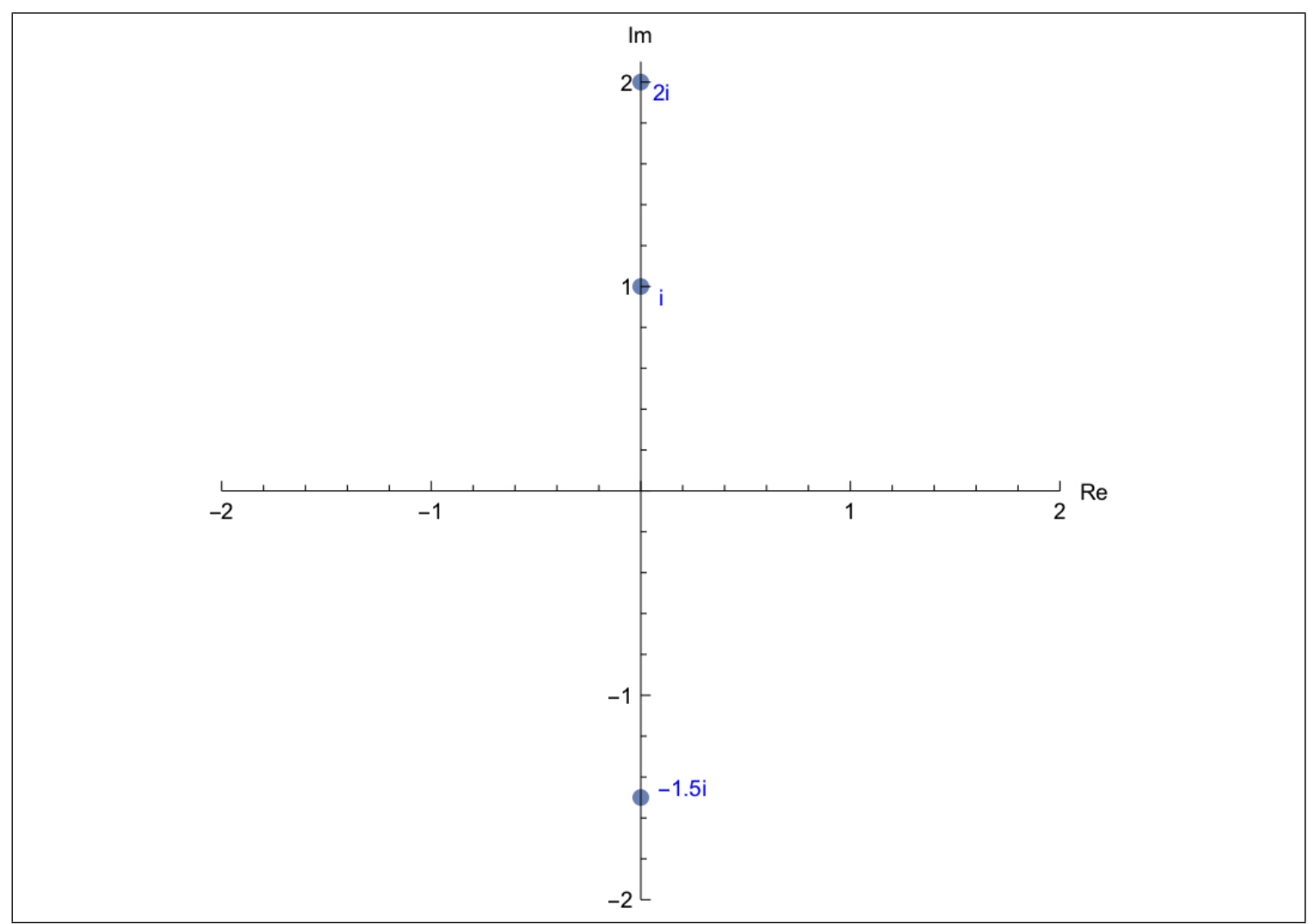

Figure 1.8: Multiples of $i$ lie along the imaginary number line

So now we can take any equation of the form $x^{2}=a$ for any real $a$, be it positive or negative, and find the solutions which are either going to be real numbers if $a$ is positive, or imaginary numbers if $a$ is negative, and we can put them on the number plane, either on the horizontal or vertical axis.

It seems like a sensible time for a definition:

\section{Definition 1.4.1. Imaginary numbers}

Imaginary numbers are real multiples of the imaginary unit $i$. They take the form $z=$ ai where $a \in \mathbb{R}$.

\subsection{The Complex Plane}

We've added an extra axis to the real number line and found ourselves in a two dimensional space. We can pin-point on here any real number and any multiple of $i$, but it feels like a great waste of space. What about the numbers which aren't on either axis?

Well, let's look at an equation like:

$$
x^{2}+2 x+2=0
$$

We are going to solve this by completing the square. This can be written as:

$$
(x+1)^{2}+1=0
$$

or

$$
(x+1)^{2}=-1
$$

But we know that the numbers which square to give -1 or $\pm i$, so it must be true that: 


$$
(x+1)= \pm i
$$

So what is $x$ ? Well, we we just use our normal rules of manipulating equations and take the 1 to the other side to give:

$$
x=-1 \pm i
$$

So what's the answer? Well, that is $x=-1 \pm i$. There's no way to simplify this any further. It's a number which has a real part to it: -1 and an imaginary part to it $\pm i$. You can check that this is a solution by plugging it back into the original equation and finding that the equation is satisfied.

One thing to note here is that by completing the square, we never found ourselves in a position where we had to take the square root of -1 . We haven't defined this object. Sometimes you will see it written that $i=\sqrt{-1}$, but we are defining $i$ instead as a number which, when squared gives -1 . We haven't needed to extend the domain of the square root function to include negative numbers.

So Where do these two numbers sit in this new two-dimensional plane? Well, at the point you get to if you move -1 along the real axis and up or down one unit along the imaginary axis:

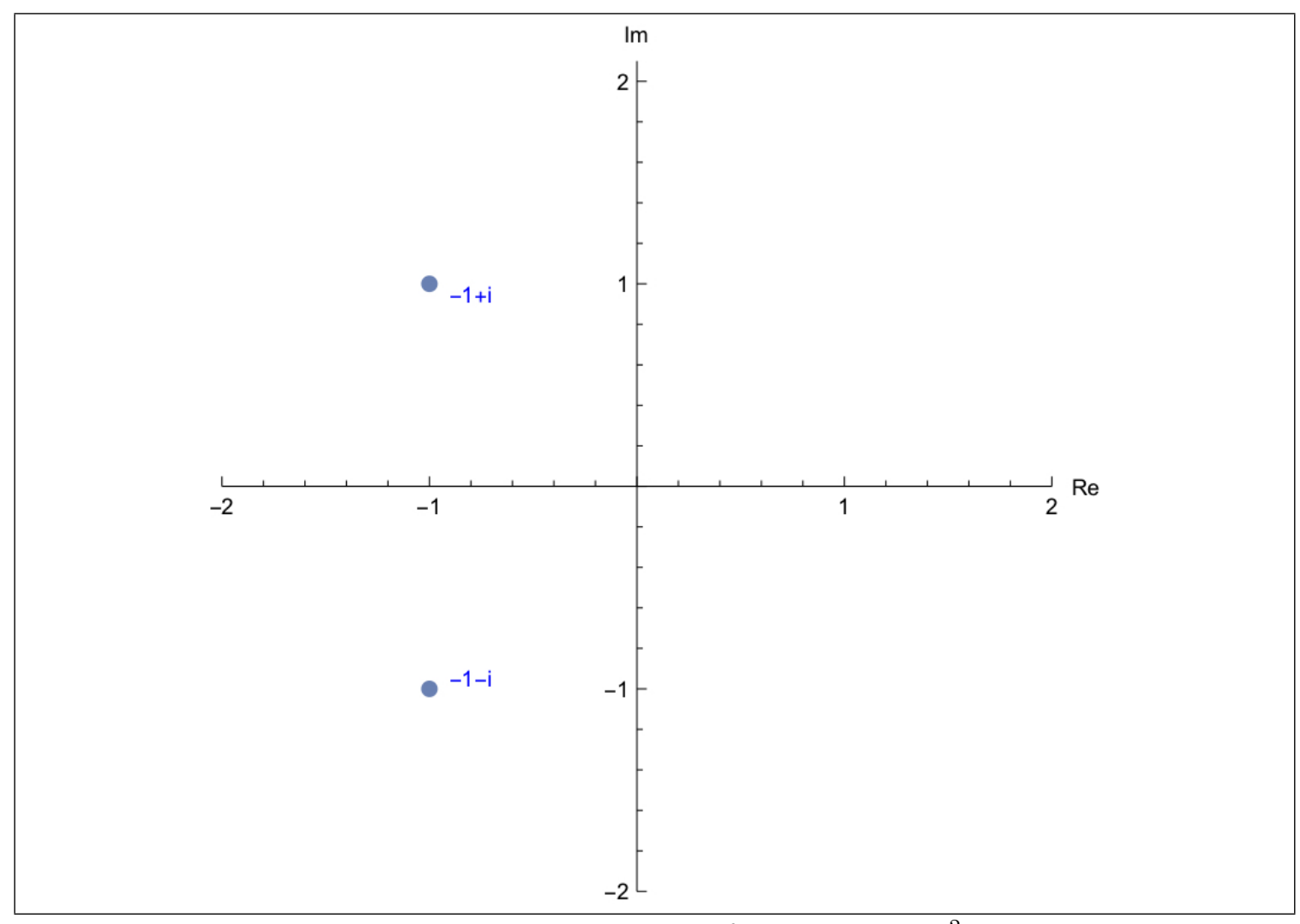

Figure 1.9: The two numbers which satisfy the equation $x^{2}+2 x+1=0$

Numbers of this form, with a real part, and an imaginary part are called complex numbers. 


\section{Definition 1.5.1. Complex numbers}

A complex number $z$ is any expression of the form $z=a+b i$, where $a$ and $b$ are real numbers and $i$ is the imaginary unit that satisfies $i^{2}=-1$.

Two complex numbers $a_{1}+i b_{1}$ and $a_{2}+i b_{2}$ are said to be equal (i.e. $\left.a_{1}+i b_{1}=a_{2}+i b_{2}\right)$ if and only if $a_{1}=a_{2}$ and $b_{1}=b_{2}$.

For a complex number $z=a+b i$, a is called the real part of $z$ and is denoted by $\boldsymbol{R e}(\boldsymbol{z})$ i.e. $\operatorname{Re}(z)=a$, while $b$ is called the imaginary part of $z$ and is denoted by $\operatorname{Im}(\boldsymbol{z})$ i.e. $\operatorname{Im}(z)=b$.

The set of all complex numbers is denoted by $\mathbb{C}$.

Note that a complex number with no imaginary part is just a real number, and so real numbers are also a special type of complex number (just with $\operatorname{Im}(z)=0$ ), and similarly a complex number with $\operatorname{Re}(z)=0$ is just an imaginary numbers.

We have a bit of a hierarchy of sets that we can think about now. Just as the integers are a subset of the real numbers we can write:

$$
\mathbb{N} \subset \mathbb{Z} \subset \mathbb{Q} \subset \mathbb{R} \subset \mathbb{C}
$$

It turns out that there are actually even bigger sets that form the totality of all "normed divisor algebras" but we definitely don't need to worry about what that means, these sets form four dimensional and eight dimensional spaces and are called the quaternions and octionions respectively.

We can think about the complex numbers as ordered pairs, just like we have $(x, y)$ coordinates, we here have the real part and the imaginary part and can write a complex number as $(\operatorname{Re}(z), \operatorname{Im}(z))$. As always there are multiple ways to write the same thing and it's useful to be able to do so in as many ways as you can.

One thing that we should notice about the two dimensional space (called The Complex Plane, or Argand Plane) is that even though the imaginary axis corresponds to imaginary numbers, we write $1,2,3$, etc. on it, and not $i, 2 i, 3 i$ etc. because the number denotes how many units of $i$ we have. Moving 1 along the imaginary axis means moving one unit of $i$.

Note that we have here written our complex numbers in a specific form: $z=a+b i$. It turns out that there are going to be other ways to write the same complex number. This particular form we call the Cartesian Form, because it will be easy to represent where the complex number is as a set of cartesian coordinates where the horizontal distance from the origin is $a$ and the vertical distance from the origin is $b$. We will later study the other ways of writing it in detail.

\section{Worked Examples 1.5.1.}

(a) Solve the following equations by completing the square where necessary. You should never need to take the square root of -1 :

(i) $x^{2}+9=0$ (ii) $x^{2}+2 x+2=0$ (iii) $x^{4}-1=0$

(b) Show that $i^{n}$, where $n$ is a non-negative integer, can only take on one of four values. 


\section{Solutions to worked examples 1.5.1:}

(a) :

To solve $x^{2}+9=0$ we write:

$$
x^{2}=-9
$$

dividing both sides by 9 and taking it inside the square we get

$$
\left(\frac{x}{3}\right)^{2}=-1
$$

But we know that the number which when squared gives -1 must be $\pm i$ and so

$$
\frac{x}{3}= \pm i \Longrightarrow x= \pm 3 i
$$

Plug it back into the formula to make sure that it does indeed solve the equation

((i) For this example we do need to complete the square:

$$
x^{2}+2 x+2=0 \Longrightarrow(x+1)^{2}+1=0 \Longrightarrow(x+1)^{2}=-1
$$

Using the same logic as in the previous example we know that the number which we've squared must be $\pm i$ and so:

$$
(x+1)= \pm i \Longrightarrow x=-1 \pm i
$$

(iii) This is a quartic equation but it's one that we can solve. First we write that:

$$
x^{4}=1
$$

which implies that $x^{2}= \pm 1$. We can deal with each of these two cases separately. For $x^{2}=1$, we just have $x= \pm 1$ and for $x^{2}=-1$ we have that $x^{2}= \pm i$, so overall the solutions are:

$$
x=-1,1, i,-i
$$

(b) Let's look at some simple cases to get the idea. If $n=1$ we have $i^{1}=i$. For $n=2$ we have $i^{2}=-1$. For $n=3$ we have $i^{3}=i * i^{2}=-i$ and for $n=4$ we have $i^{n}=i^{2} * i^{2}=-1 *-1=1$. Now we get to five and something interesting happens, we have $i^{5}=i * i^{4}=i$ which is the same as for $n=1$. In fact we can take any $n>5$ and always write $i^{n}=i^{n-4} * i^{4}=i^{n-4}$. So we can always connect an $i^{n}$ with one with a lower $n$. In fact we can subtract any number of $4 k$ 's where $k$ is an integer and $n-4 k>=0$ until we get to a $k$ such that $1 \leq n-4 k \leq 4$ and we get to one of the four values that we've seen above, ie. $i,-1,-i, 1$. Note that $n=0$ is the same as $n=4$, so we could also have shown that all values of $n$ are equivalent to $n=0,1,2,3$ rather than $n=1,2,3,4$.

\section{Check your understanding 1.5.1.}

(a) Find the value of $i^{5}$.

(b) Simplify $i^{100}$.

(c) Solve the following equations:
(i) $x^{2}+4=0$
(ii) $4 x^{2}+9=0$
(iii) $x^{2}-2 x+2=0$ 


\subsection{Simple arithmetic operations on complex numbers}

Well, now we have these new numbers as solutions to simple equations (so far just quadratic), but we want to be able to play all our usual arithmetic games with them. We're going to do this with a definition. This definition is going to be the obvious choice, but still, it's something that we have chosen.

\section{Definition 1.6.1. Addition of Complex numbers}

Given two complex numbers $z_{1}=a_{1}+i b_{1}$ and $z_{2}=a_{2}+i b_{2}$ where $a_{1}, b_{1}, a_{2}, b_{2} \in \mathbb{R}$ then:

we define their Addition as: $z_{1}+z_{2}=\left(a_{1}+i b_{1}\right)+\left(a_{2}+i b_{2}\right)=\left(a_{1}+a_{2}\right)+i\left(b_{1}+b_{2}\right)$

This, I hope seems at least reasonably sensible. If I take two complex numbers and I add them together, then the real part of the new complex number is the addition of the real parts of those that we are adding together, and similarly its imaginary part. Let's see what this looks like in the complex plane:

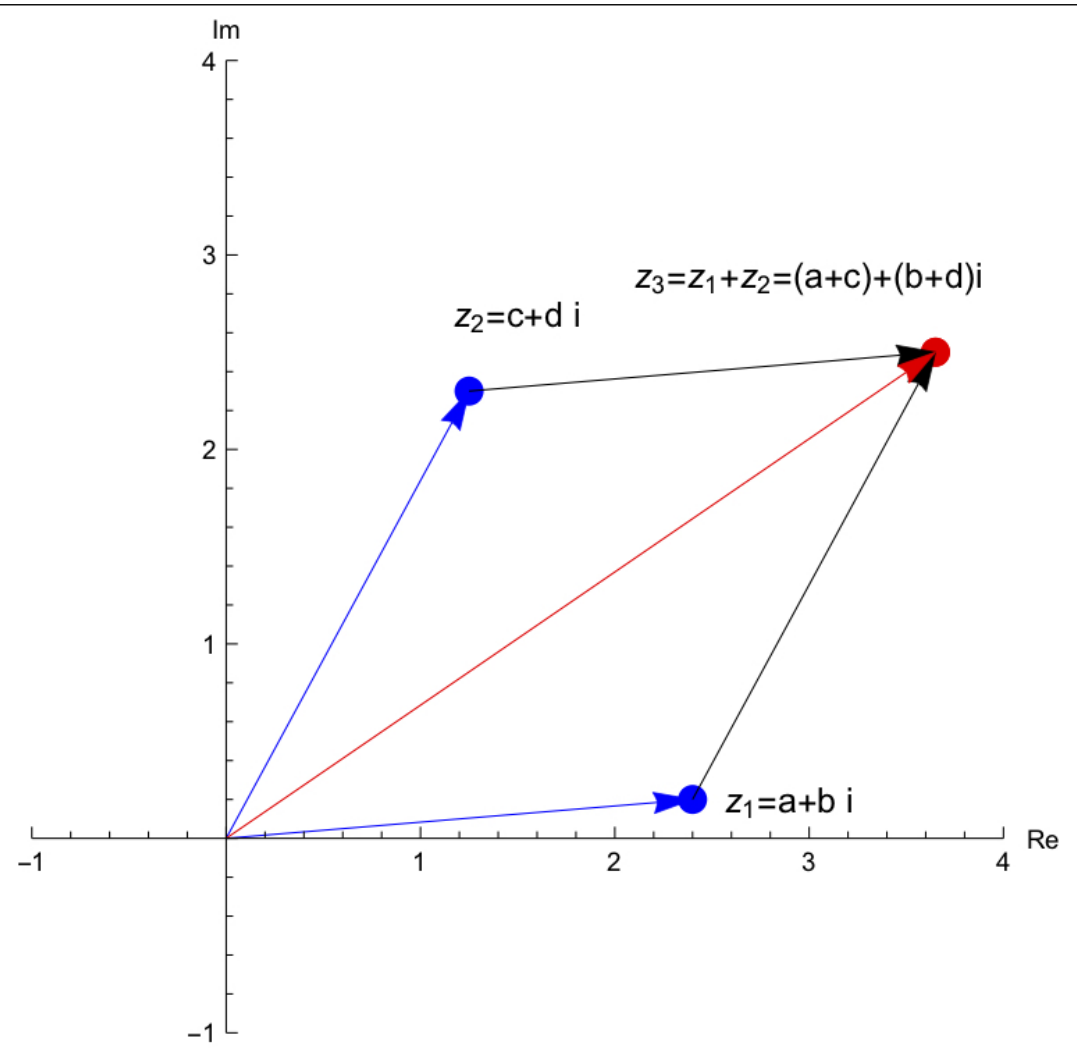

Figure 1.10: The addition of two complex numbers can be thought of as putting together the arrows that take you from the origin to each one, and stacking them to form a parallelogram. The answer in the end is the result of the two arrows. Note that the addition of real numbers follows the same rule, but the arrows are just facing in the same direction.

You see it all looks very geometrical. This means that if we add a real number to any complex number, it just shifts it left and right (depending on the sign), and if we add an imaginary number to any complex number it just shifts it up and down (depending on the sign).

To think about multiplication, we're going to start with the definition of the multiplication of two complex numbers and then we will step back and see what multiplication by a real number means in the complex plane, and then what multiplication by an imaginary number means. 


\section{Definition 1.6.2. Multiplication of Complex numbers}

Given two complex numbers $z_{1}=a_{1}+i b_{1}$ and $z_{2}=a_{2}+i b_{2}$ where $a_{1}, b_{1}, a_{2}, b_{2} \in \mathbb{R}$ then:

we define their multiplicative product as: $z_{1} z_{2}=\left(a_{1} a_{2}-b_{1} b_{2}\right)+i\left(a_{1} b_{2}+a_{2} b_{1}\right)$

This looks a bit complicated, but in fact we can see that it comes from:

$$
z_{1} z_{2}=\left(a_{1}+i b_{1}\right)\left(a_{2}+i b_{2}\right)=a_{1} a_{2}+i a_{1} b_{2}+i a_{2} b_{1}+i^{2} b_{1} b_{2}=\left(a_{1} a_{2}-b_{1} b_{2}\right)+i\left(a_{1} b_{2}+a_{2} b_{1}\right)
$$

We write $z_{1} z_{2}$ to mean the product of $z_{1}$ and $z_{2}$ just as we would do for two real numbers $x_{1}$ and $x_{2}$.

Ok, so that's a bit complicated for the moment. Let's just look at multiplication of a complex number by a real number:

Given $r \in \mathbb{R}$ and $z=a_{1}+i b_{1}$, we have:

$$
r z=r a_{1}+i r b_{1}
$$

So in terms of the complex plane this will just scale both the real and imaginary parts by a factor of $r$ towards or away from the origin depending on whether $r$ is less than or greater than 1 , and will perform a reflection about the real and imaginary axes if $r$ is negative.

Let's just think a bit more about what arithmetic operations look like in the complex plane. We are going to start with a load of random complex numbers and see what happens to them when we perform some operation. Let's start with the following complex numbers:

$$
-1,0,1,-i, i, 1+i, 1-i, 2 i
$$

In the following figure we first see these points placed in the complex plane, and then we add real and imaginary numbers to them to see how they move, and then multiply by positive and negative real numbers to see what happens. 


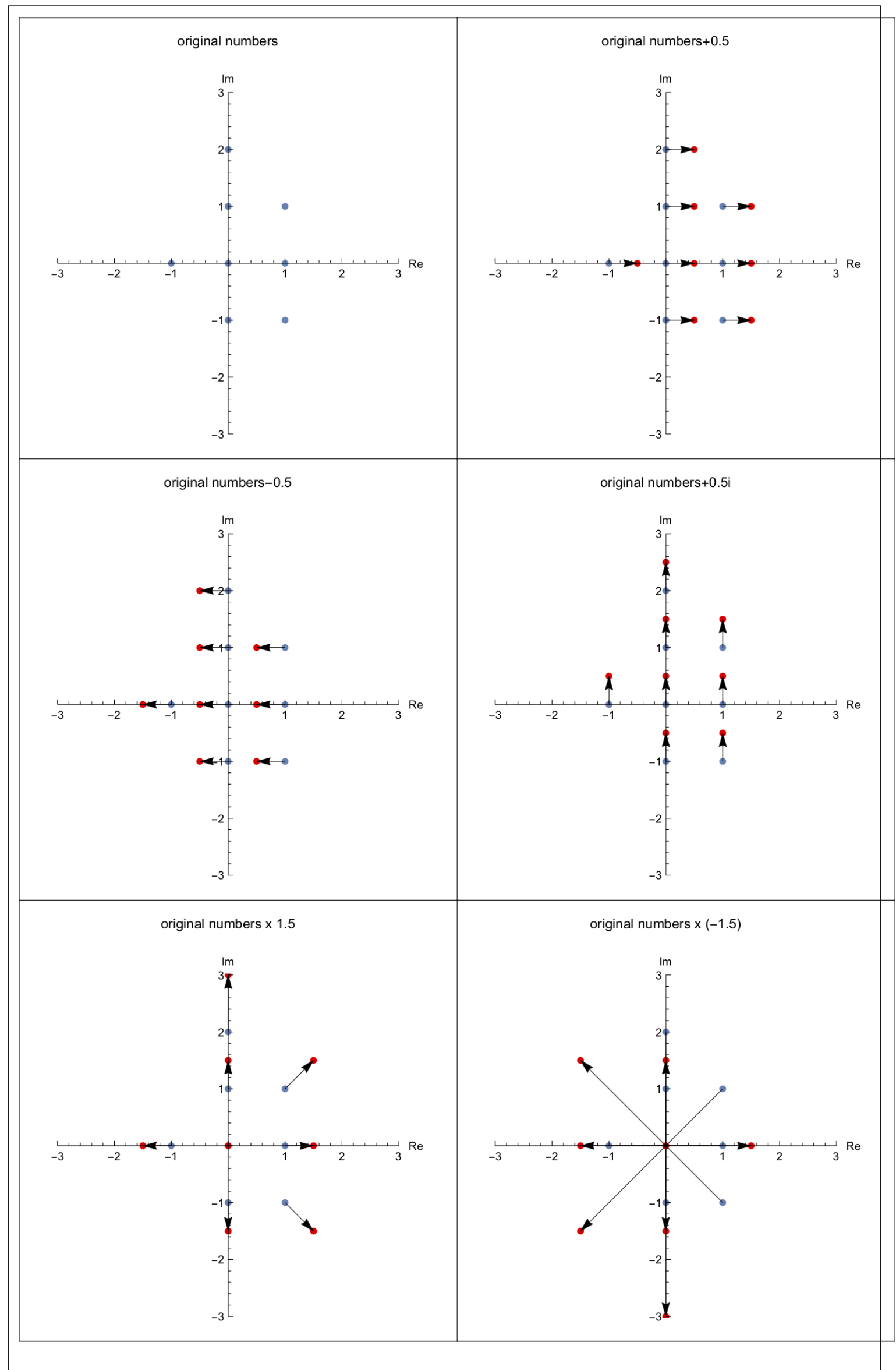

Figure 1.11: Numbers in the complex plane having added real and imaginary numbers, and multiplied by real numbers. 
What would happen to these numbers if we multiplied not be a real number but by an imaginary number?

Well, let's calculate what will happen if we multiply them all by $i$.

$$
\times i:(-1,0,1,-i, i, 1+i, 1-i, 2 i) \rightarrow(-i, 0, i, 1,-1, i-1, i+1,-2)
$$

Let's look at this mapping in the complex plane.

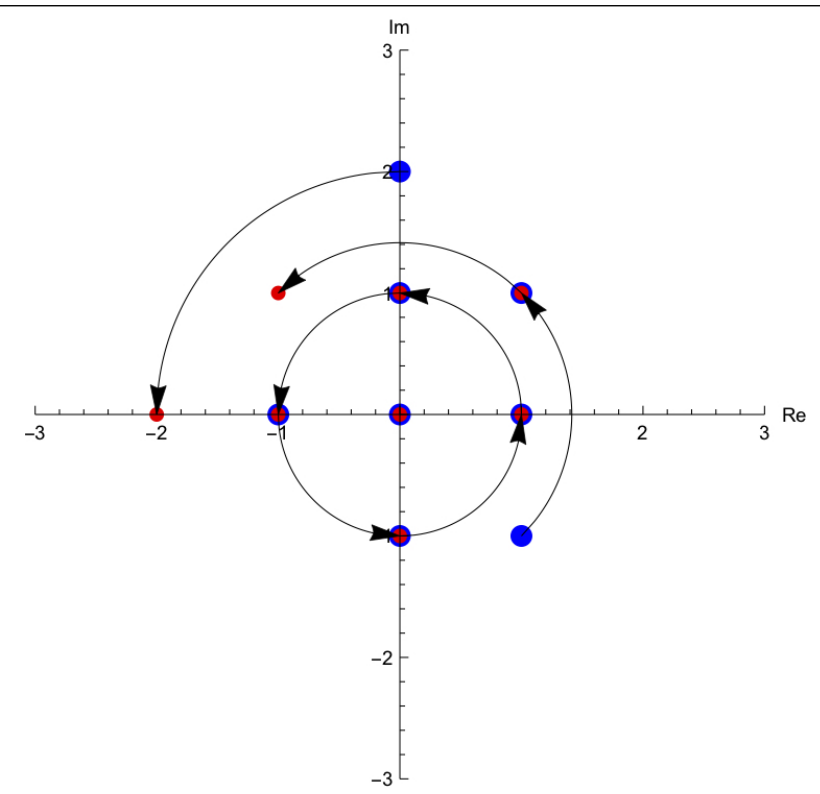

Figure 1.12: Multiplying a complex number by $i$ takes that point and rotates it by $\frac{\pi}{2}$ anticlockwise about the origin. Blue points are the original points, red points are the result of the multiplication by $i$.

We see that multiplying by $i$ corresponds to a rotation by $\frac{\pi}{2}$ radians anticlockwise about the origin. This is actually exactly what we saw before when we asked how we could multiply by -1 in two equal steps...we said that we had to do this via two rotations. Now we see the link between the arithmetic and the geometry.

What about multiplying by $2 i$ ? Well, this is the same as multiplying by $i$ and then multiplying by 2 ; so it's a rotation and a scaling. 


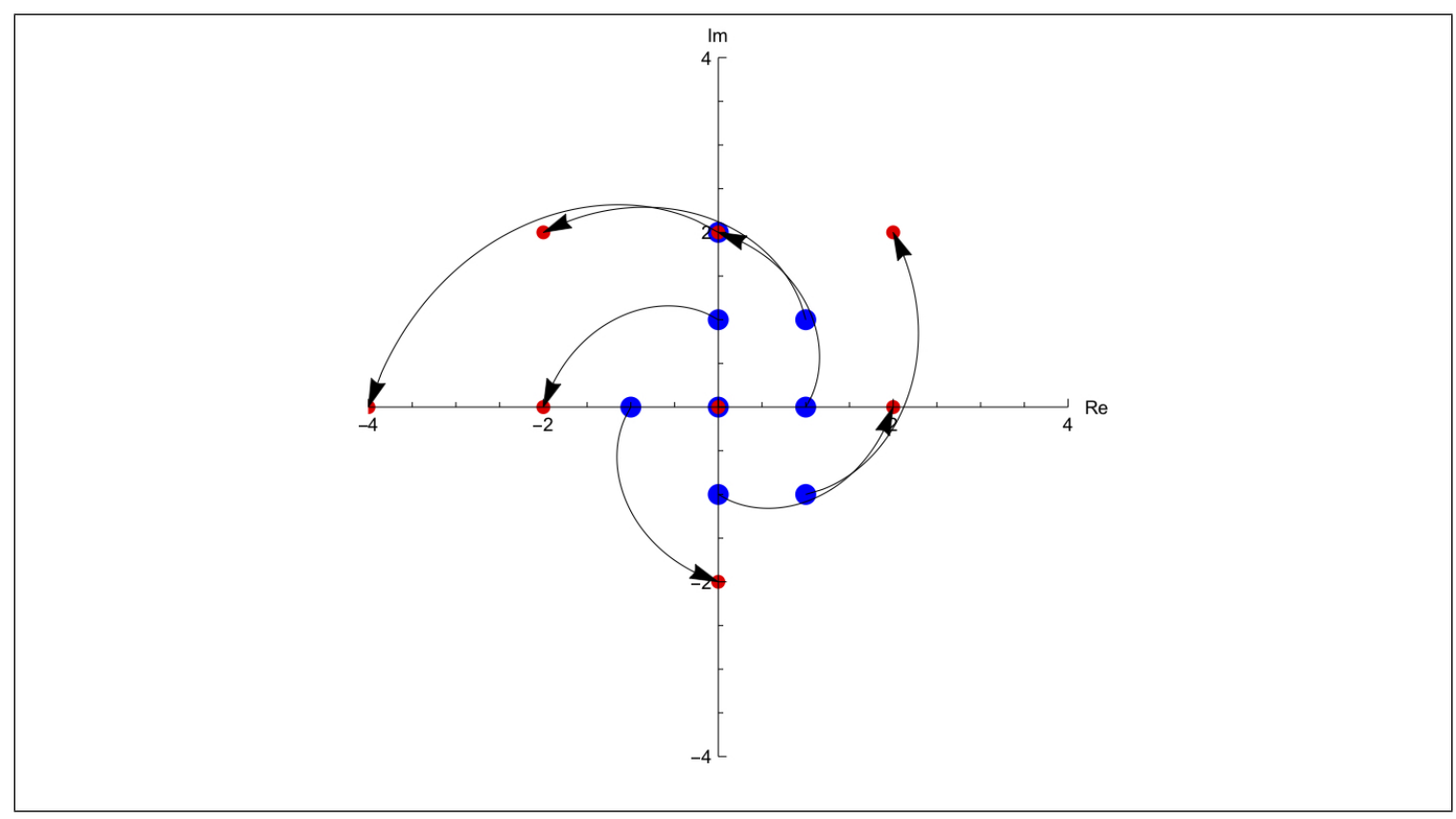

Figure 1.13: Multiplying by $2 i$ leads to a rotation and a scaling.

Now we can say that in the complex plane:

1. addition by a real number shifts left and right

2. addition by an imaginary number shifts up and down

3. addition by a complex number with both real and imaginary parts does a bit of both of the above

4. multiplication by a real number scales towards or away from the origin and reflects about the real and imaginary axis if the number is negative.

5. multiplication by an imaginary number rotates left and right and scales, and may reflect as above if it is $i$ times a negative real number.

In fact, given the above, we can think of multiplication by -1 not as reflection across the imaginary axis, but as $-1=i \times i$ therefore rotation about the origin counterclockwise by $\frac{\pi}{2}$ twice, ie. by $\pi$. Of course we could also think of it as $-1=(-i) \times(-i)$ ie. the same thing but clockwise rather than counterclockwise. 
Now let's give you something to investigate:

1. choose any number in the complex plane which is a distance 1 away from the origin, but not a simple one like 1 , or $i$, etc. You can find these by drawing a unit circle about the origin and picking any point on it.

2. Write down this number in the form $a+i b$, with $a$ and $b \in \mathbb{R}$.

3. Note that if it's on the unit circle then you should have $a^{2}+b^{2}=1$. Check that you do!

4. Work out what is the angle subtended between the positive real axis, and the line joining the origin to your number, going in the counterclockwise direction.

5. Take the numbers that we looked at before (ie. $(-1,0,1,-i, i, 1+i, 1-i, 2 i))$ and multiply them by this number.

6. What has happened to all of these numbers? Ho does this relate to the angle that you found in step 4 ?

One really important thing to note here is that:

Complex numbers are closed under addition and multiplication i.e. if $z_{1}, z_{2} \in \mathbb{C}$ then so is $z_{1}+z_{2}$ and $z_{1} z_{2}$.

This means that we don't have to worry about a different kind of number suddenly appearing. As long as we are doing regular arithmetic, so long as I give you a bunch of complex numbers, you can't find yourself in some unknown realm of numbers.

Now is a moment to step back and remember that we are in Mathemafrica, and we have the freedom to make certain choices and then explore those choices. Above we have simply defined addition and multiplication of two complex numbers. Now we can find out the consequences of this. We have to figure out what are the properties of addition and multiplication of two or more complex numbers having made the above choice.

The above definitions are what we can think of as axioms in this system. They can't be proven, they are simply the basic definitions that we've decided to give our new ingredients. Anything which comes from this in the form of properties or theorems must be proven.

Below we will postulate (this is like a guess, before we have proved something) the properties which come out of the above definitions. Each of them can be proven, and we will prove one of them below. All you need to prove them are the definitions of addition and multiplication of complex numbers along with the properties of the addition and multiplication of real numbers. 
Property 1.6.1. Common arithmetic properties of complex numbers If $z, z_{1}, z_{2}, z_{3} \in \mathbb{C}$, then:

(a) Commutative property of addition: $z_{1}+z_{2}=z_{2}+z_{1}$

(b) Commutative property of multiplication: $z_{1} z_{2}=z_{2} z_{1}$

(c) Associative property of addition: $z_{1}+\left(z_{2}+z_{3}\right)=\left(z_{1}+z_{2}\right)+z_{3}$

(d) Associative property of multiplication: $z_{1}\left(z_{2} z_{3}\right)=\left(z_{1} z_{2}\right) z_{3}$

(e) Distributive property: $\left(z_{1}+z_{2}\right) z_{3}=z_{1} z_{3}+z_{2} z_{3}$

(f) Additive Identity: $0+z=z$

(g) Multiplicative Identity: $1 z=z$

(h) Additive Inverse: $z+(-z)=0$

(i) Multiplicative Inverse: $z z^{-1}=1$ (provided $z \neq 0$ )

We will prove property 1.6.1(a) and leave the rest for homework exercises (we will frequently prove a few properties and leave the rest for Check your understanding exercises).

Property 1.6.1(a) proof: Let $z_{1}=a_{1}+i b_{1}$ and $z_{2}=a_{2}+i b_{2}$ for $a_{1}, a_{2}, b_{1}, b_{2} \in \mathbb{R}$

$$
\begin{aligned}
& z_{1}+z_{2}=\left(a_{1}+i b_{1}\right)+\left(a_{2}+i b_{2}\right) \\
& =\left(a_{1}+a_{2}\right)+i\left(b_{1}+b_{2}\right) \quad \text { Definition 1.6.1 } \\
& =\left(a_{2}+a_{1}\right)+i\left(b_{2}+b_{1}\right) \quad \text { Commutative property of addition for real numbers } \\
& =\left(a_{2}+i b_{2}\right)+\left(a_{1}+i b_{1}\right) \quad \text { Definition 1.6.1 } \\
& =z_{2}+z_{1}
\end{aligned}
$$

\section{Worked Examples 1.6.1.}

(a) Solve for $z \in \mathbb{C}$ if $z^{2}=1+4 \sqrt{3} i$. Hint: Let $z=x+i y$ for $x, y \in \mathbb{R}$

(b) Simplify the following:

(i) $(5-2 i)+(1+3 i)($ ii) $(1+i)-(3-2 i)$ (iii) $(1+2 i)(3-i)($ iv $) i(2-3 i)+(1+4 i)$ 


\section{Solutions to worked examples 1.6.1:}

(a) Letting $z=x+i y$ we have:

$$
(x+i y)^{2}=1+4 \sqrt{3} i
$$

Expanding out the left hand side gives us:

$$
x^{2}-y^{2}+2 i x y=1+4 \sqrt{3} i
$$

Make absolutely sure that you can get this! Now, for two complex numbers to be equal (ie. the left and right hand sides), their real parts and imaginary parts must separately be equal. The real part of the left is $x^{2}-y^{2}$ and that of the right is 1 , while the imaginary parts for each side are $2 x y$ for the left, and $4 \sqrt{3}$ for the right. Equating them gives us two equations:

$$
x^{2}-y^{2}=1, \quad 2 x y=4 \sqrt{3}
$$

This is two equations in two unknowns and we can solve this. Remember $x$ and $y$ are real so we are just doing regular algebra at this point. Finding the two real solutions to the above two equations and replacing them in $z=x+i y$ we have:

$$
z=-2-\sqrt{3} i, \quad z=2+\sqrt{3} i
$$

(b) In each of these cases we will just be collecting the real and imaginary parts together:

(i) $(5-2 i)+(1+3 i)=6+i$

(ii) $(1+i)-(3-2 i)=-2+3 i$

(iii) $(1+2 i)(3-i)=5+5 i$

(iv) $i(2-3 i)+(1+4 i)=2 i+3+1+4 i=4+6 i$

\section{Check your understanding 1.6.1.}

(a) By first writing $z=a+b i$ (where $a, b \in \mathbb{R}$ ) solve the equation: $z^{2}=8+6 i$

(b) Solve for the real numbers $x$ and $y$ such that $(3+2 i) x+(2-i) y=7 i$

(c) Evaluate the following expressions and write your answer in the cartesian form i.e. form $a+b i$

(i) $(5-6 i)+(3+2 i)$

(ii) $(1+2 i)-(2-3 i)$

(iii) $(2+5 i)(4-i)$

(iv) $(2+3 i)(1+i)(2-3 i)$

(v) $(1+i)-(2+3 i)(1+i)$

(vi) $(1+\sqrt{3} i)(1-\sqrt{3} i)$

(d) Show that $(a+b i)(a-b i)$ where $a, b \in \mathbb{R}$ is a real number.

(e) Find the real numbers $x$ and $y$ that satisfy the equation: $x-y+2(x+y-1) i=5+4 i$.

(f) Prove properties 1.6.1(b) to (e). 


\section{Section 2.6 checklist}

$\square$ Imaginary numbers are real multiples of $i$ where $i$ is a special number satisfying $i^{2}=-1$.

$\square$ I understand the geometric effect that multiplication by $i$ has.

$\square$ I know how to find non-real roots of an irreducible quadratic equation by completing the square.

Complex numbers are number of the form $a+b i$ where $a, b \in \mathbb{R}$ and $i$ satisfies $i^{2}=-1$.

Two complex numbers are equal if and only if their real parts and imaginary parts are equal.

$\square$ If $z=a+b i$ then $a$ is the real part of $z$ denoted by $\operatorname{Re}(z)=a$ and $b$ is the imaginary part of $z$ denoted by $\operatorname{Im}(z)=b$.

$\square$ I know how to add or multiply two complex numbers.

$\square$ Complex numbers have nice properties and I know how to use the definitions for addition and multiplication of complex numbers to prove the properties.

$\square$ I work through the worked examples before checking the solutions, and I also like doing Check Your Understanding exercises. 


\subsection{Identities, inverses and division of complex numbers}

We need to make some more definitions at this point:

\section{Definition 1.7.1. Identities and inverses}

1. The additive identity for complex numbers is a number, call it $w$, such that $z+w=$ $w+z=z$ for any complex number $z$. It becomes clear that $0+0 i$ (or simply 0$)$ is the additive identity since $z+0=z=0+z$ for any complex number $z$.

2. The additive inverse of a complex number $z$, let's again call it $w$, is a number such that if you take any number, and add $z$ to it, then add $w$ to it, you get back to the original number. We can actually write this as: $z+w=0$. ie. $w=-z$. More explicitely, given $z=a+b i$, the additive inverse is $w=-a-b i$.

3. The multiplicative identity is that number which, when you multiply it by complex number, that number remains the same. ie. if we call the multiplicative identity $w$, then for any $z \in \mathbb{C}$ we must have that $w z=z w=z$, and this number is just $w=1$.

We'll give a whole box to the next one as it definitely takes a bit more thought.

4. The other thing that we need to think about is the multiplicative inverse.

Let's first think about the multiplicative inverse for real numbers. What is the multiplicative inverse of 2 ? We call it $2^{-1}$ and it's the number that you have to multiply 2 by to get to 1 , which is clearly 0.5 . We can write this as:

$$
2^{-1} 2=1 \Longrightarrow 2^{-1}=0.5
$$

How about for complex numbers? What is the multiplicative inverse of $z=a+b i$, $a, b \in \mathbb{R}$ ? Let's call it $z^{-1}=c+d i, c, d \in \mathbb{R}$ and it must have the property such that:

$$
z z^{-1}=z^{-1} z=1
$$

In fact we only need to deal with one of $z z^{-1}$ or $z^{-1} z$ because we saw that complex numbers commute under multiplication.

Let's figure out what the $c$ and $d$ have to be in terms of $a$ and $b$. Remember, we are given $z$ and we have to figure out what is $z^{-1}$. Let's write the whole thing out:

$$
z z^{-1}=(a+b i)(c+d i)=a c-b d+i(a d+b c)
$$

and we want this to equal 1 . Well, for this to be 1 , we must have that $a c-b d=1$ and $a d+b c=0$. We can solve this for $c$ and $d$ and we find:

$$
c=\frac{a}{a^{2}+b^{2}}, \quad d=\frac{-b}{a^{2}+b^{2}}
$$

or

$$
z^{-1}=\frac{a-b i}{a^{2}+b^{2}}
$$

In fact we can therefore write:

$$
z^{-1}=\frac{1}{a+b i}=\frac{a-b i}{a^{2}+b^{2}}
$$


You might ask why we can't just write that

$$
z^{-1}=\frac{1}{a+b i}
$$

Indeed this is quite correct, but it gives you no idea about where this number is in the complex plane. In order to put it into Cartesian Form, you need to know what is the real part, and what is the imaginary part, and that is written then explicitely as:

$$
z^{-1}=\frac{a}{a^{2}+b^{2}}-\frac{b}{a^{2}+b^{2}} i
$$

or just

$$
z^{-1}=\frac{a-b i}{a^{2}+b^{2}}
$$

This way the number which is the multiplicative inverse of $z=a+b i$ is $\frac{a}{a^{2}+b^{2}}$ along the real axis and $\frac{-b}{a^{2}+b^{2}}$ along the imaginary axis.

So we can now also divide by complex numbers. Note that while the numerator is complex, the denominator is real, so if I give you a complex number, let's say $z=3-4 i$, you can find which number in the complex plane is $\frac{1}{z}$ which in this case will be

$$
\frac{3}{25}+\frac{4 i}{25}
$$

and you can pinpoint this in the complex plane. Make sure that you get this when you use the above formula for the multiplicative inverse.

Let's take an example and try and calculate:

$$
\frac{2+3 i}{3-4 i}
$$

Well, this is the same as:

$$
(2+3 i) \frac{1}{3-4 i}=(2+3 i) \frac{3+4 i}{25}=\frac{(6-12)+i(8+9)}{25}=\frac{-6+17 i}{25}
$$


Actually, we can do something kind of strange here. We can think about our first function of complex numbers. We know how to draw the graph of $y=1 / x$. We do this by setting up the $x$ and $y$ axes, and finding the points in the $(x, y)$ plane such that $y=\frac{1}{x}$. What would this mean for complex numbers. Well for real numbers, each axis was one dimensional, and so we ended up with a two dimensional graph. Here, we have a mapping from points in a two dimensional space to points in a two dimensional space, ie:

$$
f: a+b i \rightarrow \frac{a-b i}{a^{2}+b^{2}}
$$

so it kinda looks like this should be four dimensional, ie. all points $(z, w)$ such that $w=\frac{1}{z}$ Well, we can't do this, so instead we put two complex planes next to each other, and ask where entire lines of complex numbers get mapped to. The easiest lines to think about are lines of constant imaginary part (ie. horizontal lines) and lines of constant real part (ie. vertical lines). It turns out that these lines of points get mapped in a really pretty way to circles of different positions and radii under the map $z \rightarrow \frac{1}{z}$.

There is a mathemafrica blog-post here on plotting functions of complex numbers, the material is not examinable. We recommend reading this blog-post here to see some animated mapping. Below are some plots generated using Mathematica which you will also find in the blog-post.

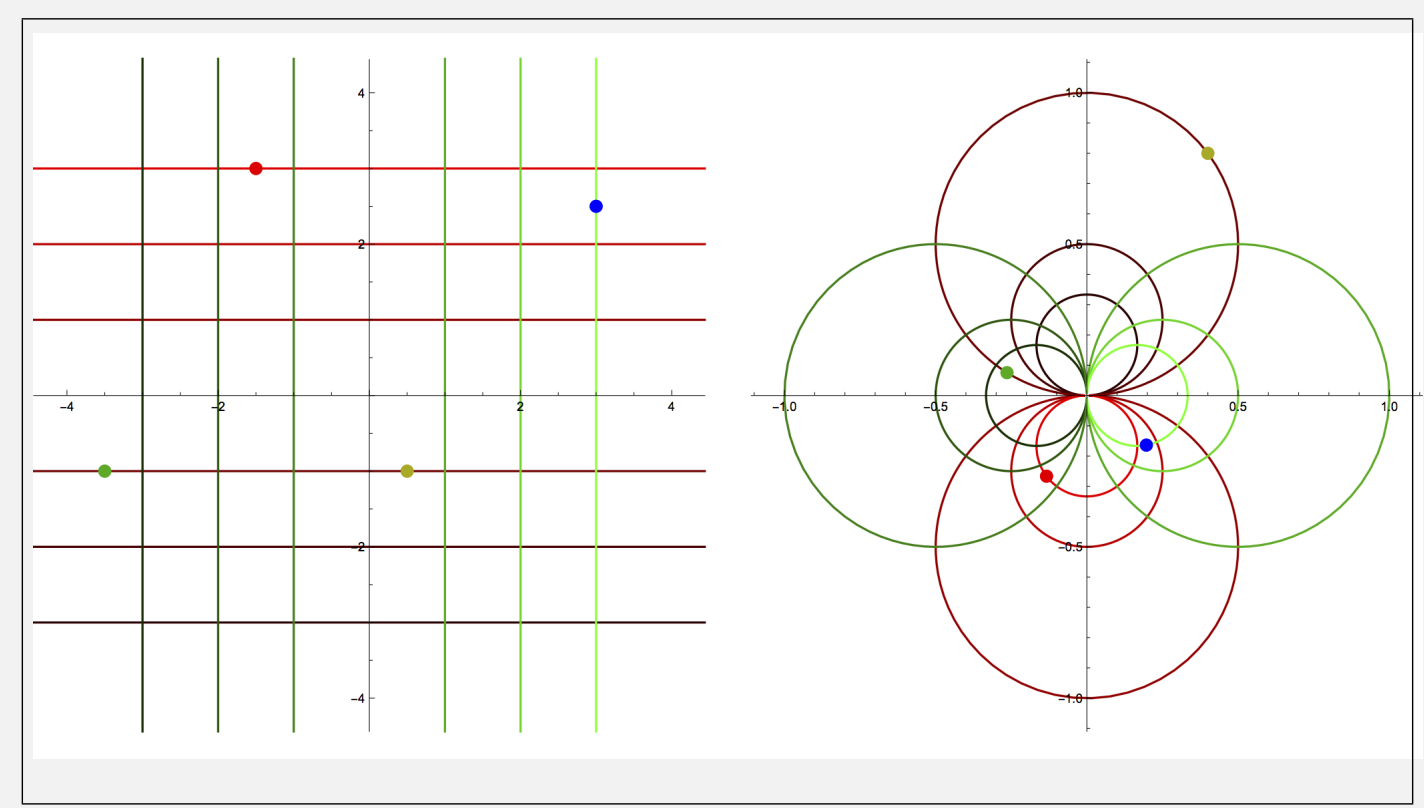

Figure 1.14: The mapping of $z \rightarrow \frac{1}{z}$ from one complex plane to its image.

In the figure above, the colors of each line are matched, and you can see four points which are mapped to their correspondingly colored points in the complex plane.

Actually, when we calculated the multiplicative inverse of $z=a+b i$ :

$$
z^{-1}=\frac{1}{a+b i}=\frac{a-b i}{a^{2}+b^{2}}
$$

we found two expressions which will be used frequently and have names of their own, they are called the complex conjugate, and the modulus respectively: 


\section{Definition 1.7.2. Complex conjugate and modulus}

(a) The complex conjugate of the complex number $z=a+b i$ is denoted by $\bar{z}$ and is defined as:

$\bar{z}=a-i b$ (simply change the sign of the imaginary part).

(b) The modulus of a complex number $z=a+b i$, denoted by $|z|$, is defined as:

$|z|=\sqrt{a^{2}+b^{2}}$

Note that the modulus of a complex number is just the distance of that number in the complex plane to the point 0 .

Remark: Geometrically, the complex conjugate $\bar{z}$ is the reflection of $z$ about the real axis, and the modulus $|z|$ is the length of a line segment joining the origin and the complex number $z$ :

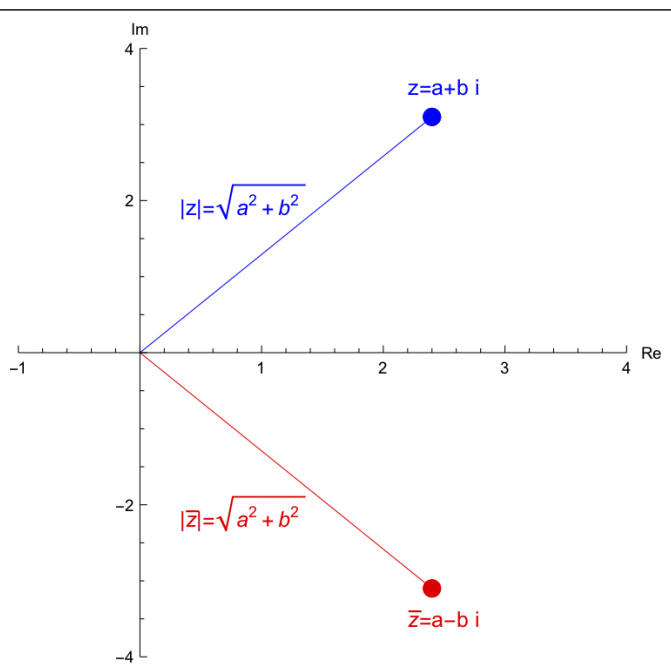

Figure 1.15: The complex conjugate of $z$ is the reflection of $z$ by the real axis, and the modulus of $z$ is the distance between the origin and the complex number $z$

Therefore, in terms of the definitions above, we have

$$
z^{-1}=\frac{a-b i}{a^{2}+b^{2}}=\frac{\bar{z}}{|z|^{2}}
$$

We can therefore write the division of complex numbers in terms of the complex conjugate and modulus:

\section{Definition 1.7.3. Division of complex numbers}

If $z, w \in \mathbb{C}, z \neq 0$, we define the quotient $\frac{w}{z}$ as follows:

$$
\frac{w}{z}=\frac{w \bar{z}}{z \bar{z}}=\frac{w \bar{z}}{|z|^{2}}
$$

So to divide by a complex number, multiply by its conjugate and divide by its modulus squared.

Remark: Complex numbers are closed under division i.e. if $z, w \in \mathbb{C}$ then $\frac{w}{z} \in \mathbb{C}$, provided $z \neq 0$. 


\section{Worked Examples 1.7.1.}

Write the following complex numbers in a cartesian form i.e. the form $z=a+i b$
(a) $\frac{1-i}{2+i}$
(b) $\frac{\overline{1+i}}{3-2 i}$
(c) $\frac{2+3 i}{i(1+i)}$

\section{Solutions to worked examples 1.7.1}

In each case here we will rationalise the denominator. This just means multiplying top and bottom by the complex conjugate:
(a) $\frac{1-i}{2+i}=\frac{(1-i)(2-i)}{(2+i)(2-i)}=\frac{1-3 i}{5}$
(b) $\frac{\overline{1+i}}{3-2 i}=\frac{1-i}{3-2 i}=\frac{5-i}{13}$
(c) $\frac{2+3 i}{i(1+i)}=\frac{2+3 i}{(i-1)}=\frac{(2+3 i)(-1-i)}{(i-1)(-1-i)}=\frac{(1-5 i)}{2}$ 


\section{Check your understanding 1.7.1.}

(a) Write the following complex numbers in a cartesian form i.e. form $z=a+b i(a, b \in \mathbb{R})$
(i) $\frac{2-3 i}{1+i}$
(ii) $\frac{\overline{3+4 i}}{1-i}$
(iii) $\frac{(1-i)^{2}}{2-3 i}$
(iv) $\frac{\overline{(2-3 i)}(1+i)}{\sqrt{3}+i}$
(v) $\frac{(1+i)^{2}}{2-\sqrt{3} i}$

(b) Find all real numbers $x$ and $y$ such that $\frac{1}{x+i y}=1+i$.

(c) If $z=2-i$, write $\frac{\bar{z}}{1-z}$ in a form $a+b i$.

(d) Find the real and the imaginary parts of

$$
\frac{1}{1+\frac{i}{1+i}}
$$

(e) Simplify $\frac{z \bar{z}^{2}}{|z|^{2}}$ if $z \in \mathbb{C}$.

(f) Let $z=1-\sqrt{3} i$. Find:
(i) $|z|$
(ii) $\overline{i z}$
(iii) $\operatorname{Re}\left(\frac{1}{z}\right)$

(g) Prove that if $(\bar{z})^{2}=z^{2}$, then $z$ is either real or pure imaginary.

(a) Give a value for $z$ and a value for $w$ for which $|z+w| \neq|z|+|w|$.

(b) Show that $z \bar{z}=|z|^{2}$.

(c) Show why the expressions in (5) and (6) are equal.

(d) Show that for any complex number $z, \operatorname{Re}(z) \leq|z|$. Hence, justify the relationship between (6) and (7).

(e) Deduce from the triangle inequality that ||$z|-| w|| \leq|z-w|$. 


\subsection{Properties of the conjugate and modulus}

Just as we did for multiplication and addition of complex numbers, we can write down the properties of the complex conjugate, all of which can be proved from its definition, and the definition of the arithmetic procedures of complex numbers:

Property 1.8.1. Properties of the complex conjugate

If $z, w \in \mathbb{C}$ and $n \in \mathbb{Z}^{+}$, then

(a) $\overline{z+w}=\bar{z}+\bar{w}$

(b) $\overline{z w}=\bar{z} \bar{w}$

(c) $\overline{\left(\frac{z}{w}\right)}=\frac{\bar{z}}{\bar{w}}$ for $w \neq 0$

(d) $z \bar{z}=|z|^{2}$

(e) $z+\bar{z}=2 \operatorname{Re}(z)$

(f) $z-\bar{z}=2 i \operatorname{Im}(z)$

(g) $\overline{\bar{z}}=z$

(h) $\overline{\left(z^{n}\right)}=(\bar{z})^{n}$

We will prove properties 1.8.1(a) and 1.8.1(h) and leave the rest for Check your understanding exercises.

Proof of property 1.8.1(a): Let $z=a+i b$ and $w=c+i d$ where $a, b, c, d \in \mathbb{R}$.

$$
\begin{aligned}
\overline{z+w} & =\overline{(a+i b)+(c+i d)} & & \\
& =\overline{(a+c)+i(b+d)} & & \text { Definition 1.6.1(a) } \\
& =(a+c)-i(b+d) & & \text { Definition 1.7.2(a) } \\
& =(a-i b)+(c-i d) & & \text { Definition 1.6.1(a) } \\
& =\overline{a+i b}+\overline{c+i d} & & \text { Definition 1.7.2(a) } \\
& =\bar{z}+\bar{w} & &
\end{aligned}
$$


Proof of property 1.8.1(h): We will prove this by mathematical induction.

Let $P(n)$ be the proposition that $\overline{\left(z^{n}\right)}=(\bar{z})^{n}$ for $n \in \mathbb{Z}^{+}$

Base Case: When $n=1$ the matter is clear.

Induction Hypothesis: Assume that $P(k)$ is true for some $k \in \mathbb{Z}^{+}$i.e. $\overline{\left(z^{k}\right)}=\overline{(z)}^{k}$.

Induction Step: We will now show that $P(k+1)$ holds true i.e. $\overline{\left(z^{k+1}\right)}=(\bar{z})^{k+1}$

$$
\begin{array}{rlrl}
\overline{\left(z^{k+1}\right)} & =\overline{\left(z^{k} z\right)} & \\
& =\overline{\left(z^{k}\right)} \bar{z} & & \text { Property 1.8.1(b) } \\
& =\bar{z}^{k} \bar{z} & & \text { By the Induction Hypothesis } \\
& =\overline{(z)}^{k+1} & &
\end{array}
$$

So $P(k+1)$ is correct. Hence by the principle of mathematical induction $P(n)$ is true for all $n \in \mathbb{Z}^{+}$.

For the modulus of a complex number we have

\section{Property 1.8.2. Properties of the modulus}

If $z$ and $w$ are complex numbers, then:
(a) $z \bar{z}=|z|^{2}$
(b) $|\bar{z}|=|z|$
(c) $|z| \geq 0$
(d) $|z|=0$ if and only if $z=0$
(e) $|z-w|$ is the distance between the points $z$ and $w$ in the complex plane.
(f) $|z w|=|z||w|$
(g) $|z|+|w| \geq|z+w|$ (The triangle inequality)

Notice that this is the first time we see inequalities in our discussion of complex numbers. This is because the quantity $|z|$ is a real number, and real numbers can be ordered. The reason why we don't discuss inequalities for complex numbers is because they cannot be "ordered". 
Real numbers possess an ordering relation i.e. they can be ordered. The axioms of order in real numbers are:

1. If $a \in \mathbb{R}$ and $a \neq 0$ then either (but not both) $a>0$ or $a<0$.

2. If $a, b \in \mathbb{R}$ and $a, b>0$, then $a b>0$.

3. If $a, b \in \mathbb{R}$ and $a, b>0$, then $a+b>0$.

Unlike real numbers, the complex numbers cannot be ordered, that is, there is no ordering satisfying axioms 1 to 3 compatible with the structure of complex numbers. For if there were such an ordering, since $i \neq 0$ then we must must have either $i>0$ or $i<0$ by (1). But if $i>0$ then $i \times i>0$ by (2), that is, $-1>0$ which is a contradiction. Similarly if $i<0$, then $-i>0$, by (2) we have $-i \times-i>0$, again $-1>0$ which is a contradiction.

The proof of the triangle inequality is probably the hardest of the basic properties to prove, so make sure that you understand every step.

Proof of Property 1.8.2(g) proof:

$$
\begin{aligned}
|z+w|^{2} & =(z+w)(\overline{z+w}) \\
& =(z+w)(\bar{z}+\bar{w}) \\
& =z \bar{z}+w \bar{z}+z \bar{w}+w \bar{w} \\
& =|z|^{2}+w \bar{z}+z \bar{w}+|w|^{2} \\
& =|z|^{2}+\overline{z \bar{w}}+z \bar{w}+|w|^{2} \\
& =|z|^{2}+2 \operatorname{Re}(z \bar{w})+|w|^{2} \\
& \leq|z|^{2}+2|z \bar{w}|+|w|^{2} \\
& \leq|z|^{2}+2|z||\bar{w}|+|w|^{2} \\
& \leq|z|^{2}+2|z||w|+|w|^{2} \\
& \leq(|z|+|w|)^{2} .
\end{aligned}
$$

\section{Worked Examples 1.8.1.}

(a) Find the distance between the given complex numbers and the origin i.e. find the modulus of $z$ if:
(i) $z=1+i$
(ii) $z=1-\sqrt{3} i$
(iii) $z=a+b-i(a-b)$

(b) Draw a sketch of the set of all $z \in \mathbb{C}$ such that:
(i) $|z|=1$
(ii) $|z-(i+1)|=2$
(iii) $|z-i|=|z+1|$
(iv) $|z+2-i| \leq 1$ 


\section{Solutions to worked examples 1.8.1:}

(a) The modulus of a complex number is its distance from the origin, so we just have to calculate that.

(i) If $z=1+i$ then $|z|=|1+i|=\sqrt{1^{2}+1^{2}}=\sqrt{2}$

(ii) If $z=1-\sqrt{3} i$ then $|z|=|1-\sqrt{3} i|=\sqrt{1^{2}+\sqrt{3}^{2}}=\sqrt{1+3}=2$

(iii) If $z=a+b-i(a-b)$ then $|z|=|a+b-i(a-b)|=\sqrt{(a+b)^{2}+(a-b)^{2}}=\sqrt{2\left(a^{2}+b^{2}\right)}$

(b) Draw a sketch of the set of all $z \in \mathbb{C}$ such that:

(i) $|z|=1$. That is, which points in the complex plane are a distance 1 from the origin? This is just the unit circle.

(ii) $|z-(i+1)|=2$. Remember that the modulus of $z-w$ is the distance between the complex numbers $z$ and $w$ in the complex plane, so this is asking for all points $z$ which are a distance 2 away from $(1+i)$, that is a circle of radius 2 about the point $(1+i)$.

(iii) $|z-i|=|z+1|$. This is asking for those points $z$ which are the same distance away from $i$ as they are from the point -1 . Of course the point right in between these points is one such $z$ but in fact there is a whole line of them as can be seen in the figure

(iv) $|z+2-i| \leq 1$. This is all points $z$ less than 1 unit away from the point $(-2+i)$, which corresponds to a unit disk centred on the points $(-2+i)$.

Each of the above could also have been solved by setting $z=a+b i$ and expanding the modulus, then equating real and imaginary parts of both sides. This would give two equations in the two unknowns $a$ and $b$ which can be solved for. Try this and make sure that you get the same answers.

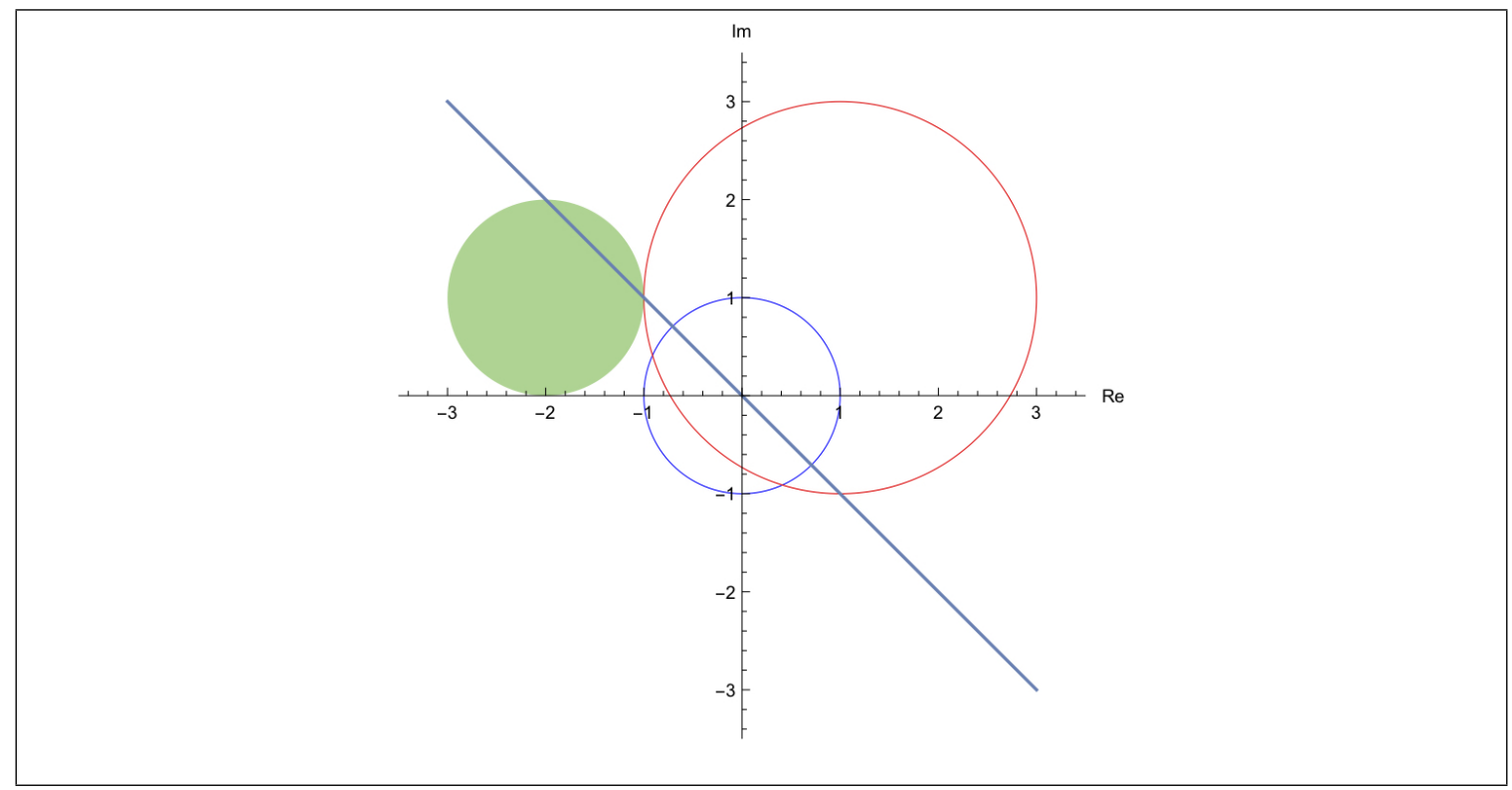

Figure 1.16: Solutions to the above questions: b) i)Blue, ii)Red, iii) Black, iv) Green 


\section{Check your understanding 1.8.1.}

(a) Find the modulus of the following complex numbers:

(i) $2-5 i$ (ii) $-2 i$ (iii) $2+\pi$ (iv) $-1+2 \sqrt{2} i$ (v) $-1-\sqrt{2}$

(b) If $w=1-2 i$ and $z=2+3 i$, find ||$w|-\bar{z}|$.

(c) Describe and sketch the following subsets of the complex plane:

(i) $\{z \in \mathbb{C}|5<| 5 z+10 i \mid<10\}$

(ii) $\{z \in \mathbb{C}|| \bar{z}+4|\geq| 2+i z \mid\}$

(iii) $\{z \in \mathbb{C}|| z-(1+2 i) \mid=1\}$

(iv) $\{z \in \mathbb{C} \mid \operatorname{Re}(z)+\operatorname{Im}(z) \geq 0\}$

(v) $\{z \in \mathbb{C}|| z+i|=| z+1 \mid\}$

(vi) $\{z \in \mathbb{C}|| z+i \mid \leq 1$ and $|z+1| \leq 1\}$

(d) Show that the complex numbers satisfying $\operatorname{Re}\left(\frac{1}{z}\right)=\frac{1}{2}$ where $z \in \mathbb{C}$ form a circle. Give it's center and radius.

(e) If $z=a+b i$, where $a, b \in \mathbb{R}, b \neq 0$, and $|z|=1$, show that

$$
\frac{z-1}{z+1}
$$

is purely imaginary.

(f) If $z+\frac{1}{z}$ is real, where $z \in \mathbb{C}$, prove that $|z|=1$ or $z$ is real.

(g) Prove Properties 1.8.1 and 1.8.2

(h) The definition of the relation $>$ in the real number system is based upon the existence of a subset $\mathscr{P}$ (the positive reals) having the following properties:

(i)For any real number $\alpha \neq 0$, either $\alpha$ or $-\alpha$ (but not both) belongs to $\mathscr{P}$.

(ii) If $\alpha$ and $\beta$ belong to $\mathscr{P}$, so does $\alpha+\beta$.

(iii) If $\alpha$ and $\beta$ belong to $\mathscr{P}$, so does $\alpha \times \beta$.

When such a set $\mathscr{P}$ exists we write $\alpha>\beta$ if and only if $\alpha-\beta$ belongs to $\mathscr{P}$. Prove that the complex number system does not possess a non-empty subset $\mathscr{P}$ having properties (i),(ii), and (iii).

\section{Section 2.8 Checklist}

$\square$ I know what a complex conjugate of $z=a+b i$ is and how it is defined, i.e. $\bar{z}=a-b i$.

$\square$ I know the geometric interpretation of the complex conjugate i.e. reflection of $z$ about the real axis.

$\square$ I know how to use the complex conjugate to write a quotient of two complex number in a Cartesian form i.e. form $a+b i$.

$\square$ On the complex plane, the modulus of a complex number is the distance between a complex number $z=a+b i$ and the origin, it is defined as $|z|=\sqrt{a^{2}+b^{2}}$.

$\square$ I understand why complex numbers cannot be ordered.

$\square$ I know how to sketch subsets of the complex plane. 


\subsection{The argument of a complex number}

Here we are going to give the second way of representing a complex number. Every number sits at some point in the complex plane and every point in the complex plane corresponds to a unique complex number. So far we have written these points by their Cartesian representation. ie. what is the real part of the complex number and what is the imaginary part. This representation is one-to-one. I give you a point in the complex plane and you can give me $a+b i$, and vice versa.

Here we're going to see that we can represent them in a different way in the complex plane.

This representation will be written not in terms of $a$ and $b$ denoting the real and imaginary parts, but in terms of the distance of the point in the complex plane from the origin, and the angle subtended between the positive real axis and the line joining the origin to the complex number.

What's going to be very strange here is that this representation will not be one-to-one.

Let's take an example here. Given the complex number $z=1+i$, we can ask what angle $\theta$ does the line between 0 and $z$ make with the positive real axis (going anti-clockwise)? Let's look at this in the complex plane:

For instance, given the complex number $z=1+i$, what angle $\theta$ does it make with the positive real axis, and how far is it from the origin?

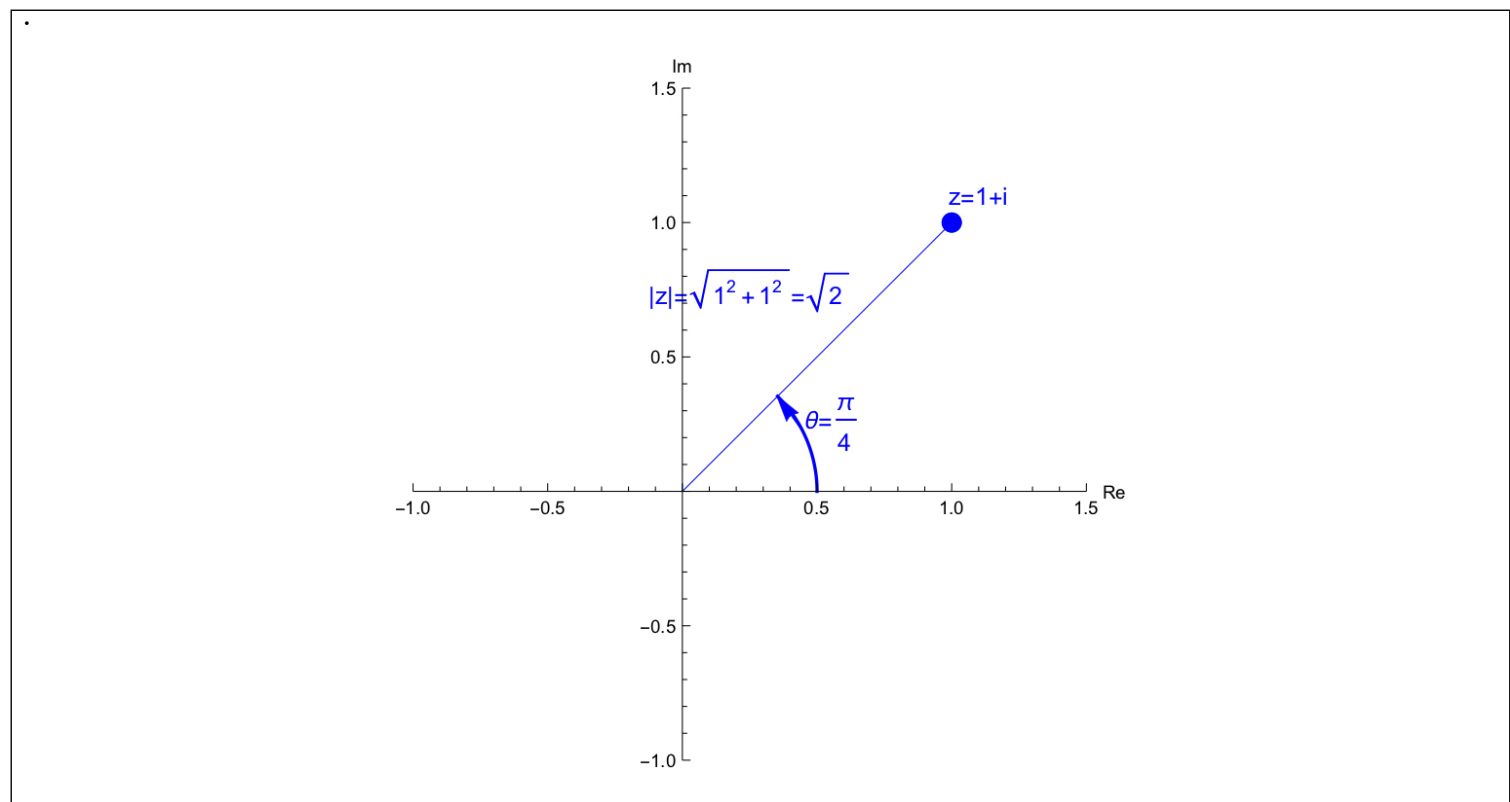

Figure 1.17: A complex number $z=1+i$ can be located in the complex plane using its modulus $|1+i|=\sqrt{2}$ and the angle theta read counterclockwise from the positive real axis.

This complex number is $\sqrt{2}$ units away from the origin, and makes an angle of $\theta=\frac{\pi}{4}$ counterclockwise from the positive real axis.

What this means is that if I had simply told you to locate the number in the complex plane which is modulus $\sqrt{2}$ and makes angle $\frac{\pi}{4}$ with the positive real axis, you would be able to uniquely pinpoint where this number was and you could tell me that it has real part 1 and imaginary part 1. ie it is $z=1+i$.

This tells us that there is a mapping from $(|z|, \theta) \rightarrow(\operatorname{Re}(z), \operatorname{Im}(z))$.

The angle theta is called an argument of the complex number. What's strange is that while giving $|z|$ and $\theta$ will give me a unique point in the complex plane, there are an infinite number of different $\theta$ 's which correspond to the same point. The reason for this is that, for instance with $z=1+i$, although we went counterclockwise from the positive real axis an angle $\frac{\pi}{4}$, we could also have gone $\frac{\pi}{4}+2 \pi$ and we would have ended up at the same place: 


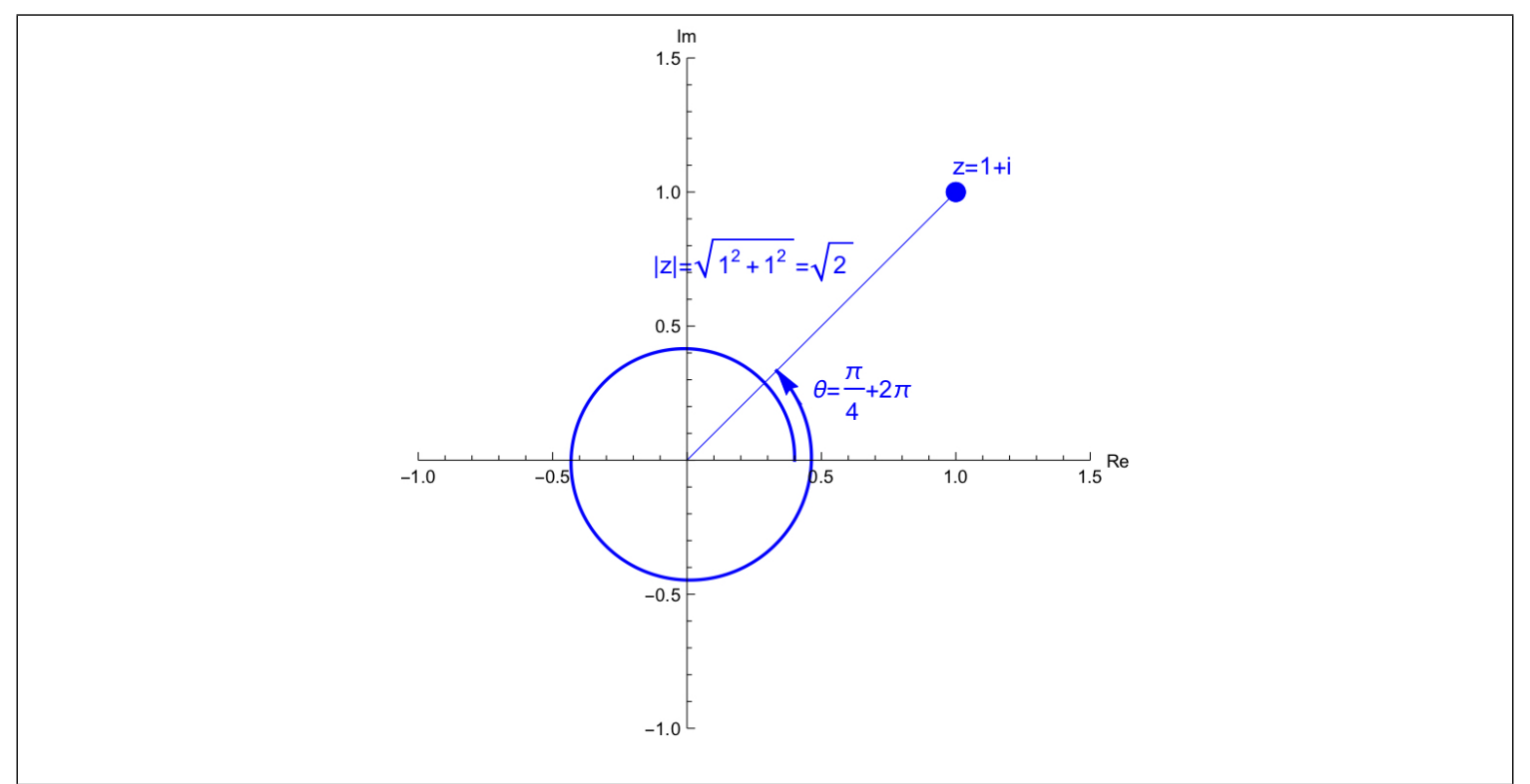

Figure 1.18: You could have gone round a full circle plus $\frac{\pi}{4}$ and arrived at the same point as just going $\frac{\pi}{4}$.

In fact we could have gone round any number of full rotations. This tells us that if we said where is the number with modulus $\sqrt{2}$ and with angular position $2 \pi+\frac{\pi}{4}$ it would also be $z=1+i$. Thus, this representation is not one-to-one with the points in the complex plane.

Here we can define the argument of a complex number as follows:

\section{Definition 1.9.1. An argument of a complex number $z$}

An argument of a non-zero complex number $z$, denoted by $\arg (z)$, is an angle $\theta$ measured from the positive real axis to the line segment joining $z$ to the origin.

The set of all possible arguments can be written as:

$$
\arg (z)=\operatorname{Arg}(z)+2 \pi k, \quad k \in \mathbb{Z}
$$

where $\operatorname{Arg}(z)$ is called the principal value of the argument satisfying $-\pi<\operatorname{Arg} \leq \pi$. This means that $\arg (z)$ is an infinite set, and $\operatorname{Arg}(z)$ is a single number.

You might ask why we are introducing another way of denoting complex numbers where the Cartesian Form seemed just fine, thank you very much. Well, it turns out that many things will be much easier in the language of the modulus and the argument as we will see in the following sections.

We want to be able to calculate $\operatorname{Arg}(z)$ wherever $z$ is in the complex plane. In oder to do this it depends on which quadrant of the complex plane the number is in.

$$
\begin{aligned}
& \text { If } z=a+b i \text { and } \theta=\arctan \left(\frac{b}{a}\right) \text { then } \\
& \qquad \operatorname{Arg}(z)= \begin{cases}\theta & \text { If } z \text { is in the first or fourth quadrants } \\
\theta+\pi & \text { If } z \text { is in the second quadrant } \\
\theta-\pi & \text { If } z \text { is in the third quadrant }\end{cases}
\end{aligned}
$$




\section{Worked Examples 1.9.1.}

Plot the following numbers in the complex plane and find $|z|, \operatorname{Arg}(z)$ and $\arg (z)$ for:
(a) $z=1+i$
(b) $z=1-i$
(c) $z=-\sqrt{3}-i$
(d) $z=-1+\sqrt{3} i$
(e) $z=i$
(f) $z=2$
(g) $z=-\pi$

Solutions to worked examples 1.9.1:

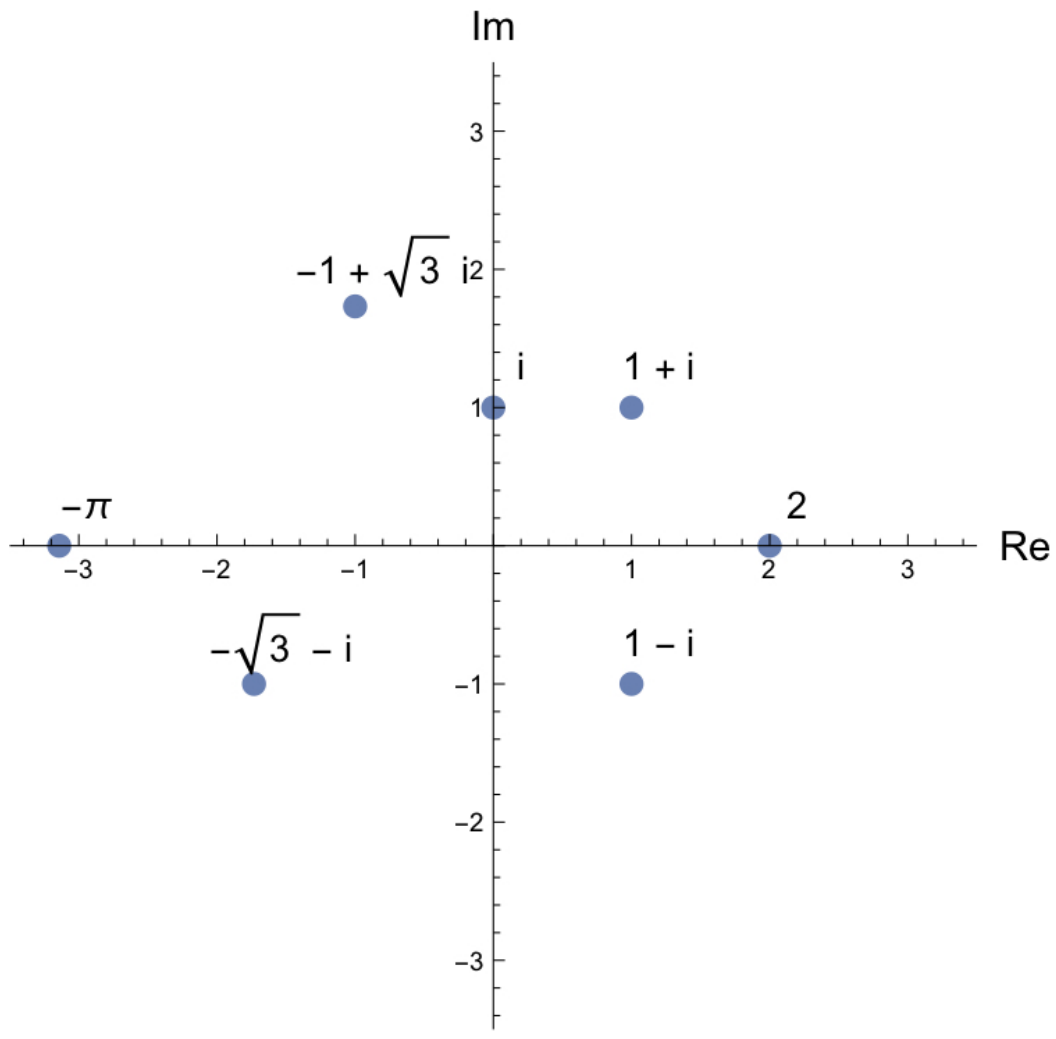

Figure 1.19: positions of the points listed above.
(a) For $z=1+i,|z|=\sqrt{1^{2}+1^{2}}=\sqrt{2}, \operatorname{Arg}(z)=\frac{\pi}{4}, \arg (z)=\left\{\frac{\pi}{4}+2 \pi k \mid k \in \mathbb{Z}\right\}$
(b) $z=1-i,|z|=\sqrt{1^{2}+(-1)^{2}}=\sqrt{2}, \operatorname{Arg}(z)=-\frac{\pi}{4}, \arg (z)=\left\{-\frac{\pi}{4}+2 \pi k \mid k \in \mathbb{Z}\right\}$
(c) $z=-\sqrt{3}-i|z|=\sqrt{(-\sqrt{3})^{2}+(-1)^{2}}=2, \operatorname{Arg}(z)=-\frac{5 \pi}{6}, \arg (z)=\left\{-\frac{5 \pi}{6}+2 \pi k \mid k \in \mathbb{Z}\right\}$
(d) $z=-1+\sqrt{3} i|z|=\sqrt{(-1)^{2}+(\sqrt{3})^{2}}=2, \operatorname{Arg}(z)=\frac{2 \pi}{3}, \arg (z)=\left\{\frac{2 \pi}{3}+2 \pi k \mid k \in \mathbb{Z}\right\}$
(e) $z=i,|z|=\sqrt{1^{2}}=1, \operatorname{Arg}(z)=\frac{\pi}{2}, \arg (z)=\left\{\frac{\pi}{2}+2 \pi k \mid k \in \mathbb{Z}\right\}$
(f) $z=2,|z|=\sqrt{2^{2}}=2, \operatorname{Arg}(z)=0, \arg (z)=\{2 \pi k \mid k \in \mathbb{Z}\}$ 
(g) $z=-\pi,|z|=\sqrt{(-\pi)^{2}}=\pi, \operatorname{Arg}(z)=\pi, \arg (z)=\{\pi+2 \pi k \mid k \in \mathbb{Z}\}$

\section{Check your understanding 1.9.1.}

(a) Find $\operatorname{Arg}(z)$ and $\arg (z)$ if:
(i) $\sqrt{3}+i$
(ii) $1-\sqrt{3} i$
(iii) $8 i$
(iv) $-1+i$
(v) $-3-3 i$

(b) Sketch the following subsets of the complex plane
(i) $\left\{z \in \mathbb{C}: 0 \leq \arg (z) \leq \frac{\pi}{2}\right\}$
(ii) $\left\{z \in \mathbb{C}: \frac{\pi}{4} \leq \arg (z) \leq \frac{\pi}{2}\right.$ and $\left.2<|z| \leq 3\right\}$

\section{Section 2.9 Checklist}

$\square$ I know what an argument of a complex number is.

$\square$ I understand that a complex number $z$ has infinitely many arguments $\arg (z)$ but has a unique principal value on $(-\pi, \pi]$ denoted by $\operatorname{Arg}(z)$.

$\square$ I know how to find $\operatorname{Arg}(z)$ of a complex number $z$. 


\subsection{The Modulus-Argument form of a complex number}

We've seen now that you can use $|z|$ and $\arg (z)$ to pinpoint a number in the complex plane. Now we are going to discover how to write $z$ explicitly using these quantities

Consider the diagram below:

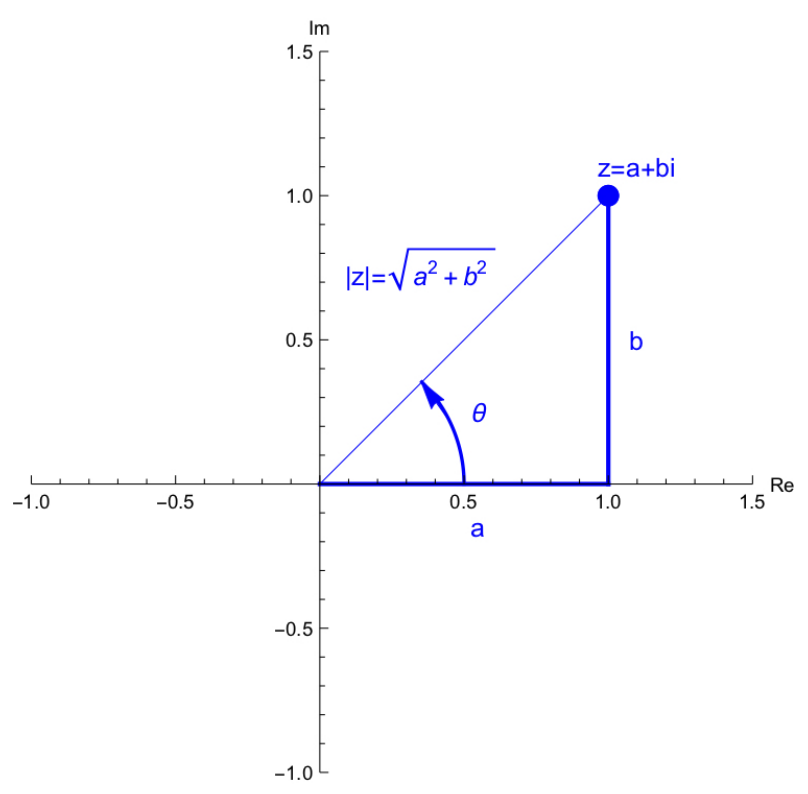

Figure 1.20: A complex number $z$ located $|z|$ away from the origin with real part $a$ and imaginary part $b$ making an angle of $\theta$ with the positive real axis

It's clear using our basic trigonometry that we can relate many of these quantities. In particular:

$$
\sin \theta=\frac{b}{|z|} \Rightarrow b=|z| \sin \theta
$$

and

$$
\cos \theta=\frac{a}{|z|} \Rightarrow a=|z| \cos \theta
$$

But this means that we can write:

$$
z=|z| \cos \theta+i|z| \sin \theta=|z|(\cos \theta+i \sin \theta)
$$

There's a specific name for this form:

Definition 1.10.1. Modulus-Argument form of a complex number

If $z$ is a non-zero complex number and $\theta$ is an argument of $z$, then the expression

$$
z=|z|(\cos \theta+i \sin \theta)
$$

is called the modulus-argument form of $z$.

It is usual to choose $\theta$ to be the principal argument of the $z$.

The modulus-argument form is also known as the polar form. 


\section{Worked Examples 1.10.1.}

Write the following complex numbers in the modulus-argument form.
(a) $z=1+i$
(b) $z=1-i$
(c) $z=-\sqrt{3}-i$
(d) $z=-1+\sqrt{3} i$

\section{Solutions to worked examples 1.10.1:}

In each of these cases we just have to use that $|z|=\sqrt{\operatorname{Re}(z)^{2}+\operatorname{Im}(z)^{2}}$ and trigonometry to calculate the principle argument. Here we plot each of the numbers in the complex plane:

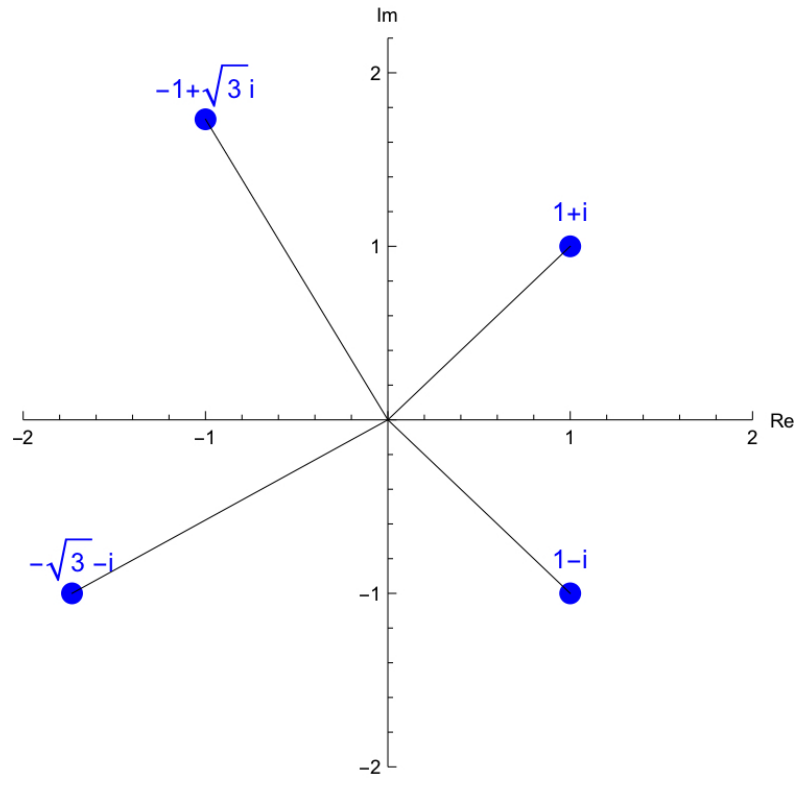

Figure 1.21: The positions of the complex numbers whose modulus and argument we have to calculate

Looking at the first one, the argument is just the angle subtended between the positive real axis and the line joining the origin to the number. We see that this is a triangle with base 1 and height 1 , and thus the angle is $\arctan (1)=\frac{\pi}{4}$. Then length of the hypotenuse is $\sqrt{1^{2}+1^{2}}=\sqrt{2}$. Thus we can write:

$$
1+i=\sqrt{2}\left(\cos \left(\frac{\pi}{4}\right)+i \sin \left(\frac{\pi}{4}\right)\right)
$$

For the next number the angle is the same, but just measured in the opposite direction (ie. clockwise) and is therefore negative the previous one. So:

$$
1+i=\sqrt{2}\left(\cos \left(-\frac{\pi}{4}\right)+i \sin \left(-\frac{\pi}{4}\right)\right)
$$

For the next number we have to be a bit more careful with the calculation of the angle. Both parts are negative, and so we can measure the angle between the negative real axis and the line between the origin and the number, and then add on to that $\pi$. 


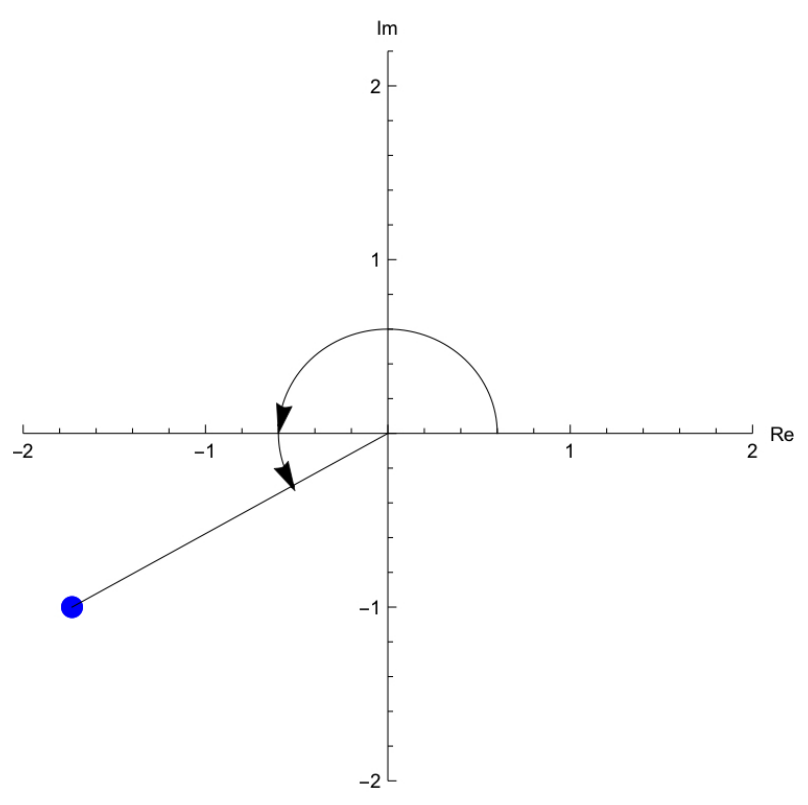

Figure 1.22: The positions of the complex numbers whose modulus and argument we have to calculate

So this argument is: $\pi+\arctan \left(\frac{1}{\sqrt{3}}\right)=\pi+\frac{\pi}{6}=\frac{7 \pi}{6}$, and therefore (having calculated that $\sqrt{(-\sqrt{3})^{2}+(-1)^{2}}=2$ :

$$
-\sqrt{3}-i=2\left(\cos \frac{\pi}{7}+i \sin \frac{\pi}{7}\right)
$$

Note that when we defined the principle argument we actually said that it had to be between $\pi$ and $\pi$ but sometimes we will choose to represent the argument in the range $[0,2 \pi)$.

For the last example, you should get:

$$
-\sqrt{3}-i=2\left(\cos \frac{2 \pi}{3}+i \sin \frac{2 \pi}{3}\right)
$$

\section{Check your understanding 1.10.1.}

Write the following complex numbers in the modulus-argument form.
(a) $-\sqrt{3}+i$
(b) $5-5 \sqrt{3} i$
(c) $-3-3 i$
(d) $\sqrt{3}+i$
(e) $-1+i$

\section{Section 2.10 Checklist}

$\square$ Given a complex number $z$, I know how to find $|z|$ and $\operatorname{Arg}(z)$.

$\square$ I know how to use $|z|$ and $\operatorname{Arg}(z)$ to write $z$ in a modulus argument form. 


\subsection{Multiplication and de Moivre's Theorem}

Remember when we first saw the basic arithmetic operations on complex numbers? Addition in Cartesian form was really easy, but multiplication, which had this lovely geometric interpretation (it's just rotations) was not so simple. Remember in Cartesian form: $(a+b i)(c+d i)=(a c-b d)+$ $(a d+b c) i$ which is just a bit messy. It turns out that in modulus argument form, multiplication is super simple!

Theorem 1.11.1. Multiplication of two complex numbers in the modulusargument form

Suppose that $z=|z|(\cos \alpha+i \sin \alpha)$ and $w=|w|(\cos \beta+i \sin \beta)$ are non-zero complex numbers in the modulus-argument form, then their product is:

$$
z w=|z||w|(\cos (\alpha+\beta)+i \sin (\alpha+\beta))
$$

Remark: This theorem gives us the geometric interpretation for multiplication of complex numbers, i.e. multiplying two complex numbers is equivalent to multiplying their moduli and adding their arguments.

The proof of the theorem is as follows:

\section{Proof of Theorem 1.11.1}

$$
\begin{array}{rlr}
z w & =(|z|(\cos \alpha+i \sin \alpha))(|w|(\cos \beta+i \sin \beta)) & \text { Modulus-argument form } \\
& =|z||w|(\cos \alpha+i \sin \alpha)(\cos \beta+i \sin \beta) & \text { Commutative property } \\
& =|z||w|(\cos \alpha \cos \beta+i \cos \alpha \sin \beta+i \cos \beta \sin \alpha-\sin \alpha \sin \beta) & \text { Definition 1.6.2 } \\
& =|z||w|((\cos \alpha \cos \beta-\sin \alpha \sin \beta)+i(\cos \alpha \sin \beta+\cos \beta \sin \alpha)) & \text { Definition 1.6.1 } \\
& =|z||w|(\cos (\alpha+\beta)+i \sin (\alpha+\beta)) & \text { Compound angle identities }
\end{array}
$$

and visually, we can see an example of the product of two complex numbers here: 


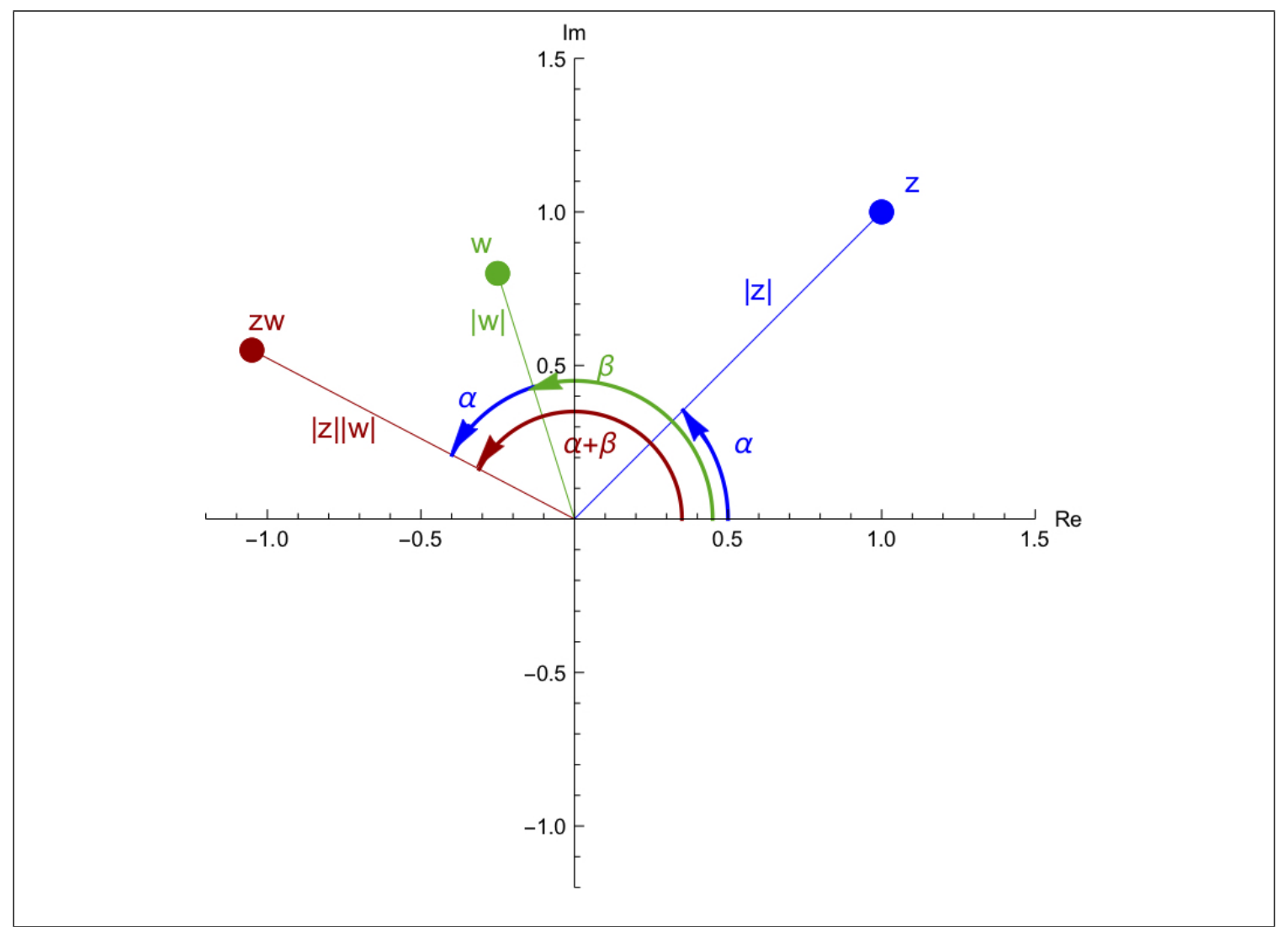

Figure 1.23: Here we have two complex numbers $z$ and $w$ with their moduli $(|z|$ and $|w|)$ and arguments $(\alpha$ and $\beta$ ) written down. The product of them $z w$ is given by a complex number whose modulus is $|z||w|$ and whose argument is $\alpha+\beta$.

This is something really fundamental to understand and remember:

In modulus argument form, when you multiply together two complex numbers, the moduli multiply and the arguments add. 
How would you write down the complex number $i$ in modulus argument form?

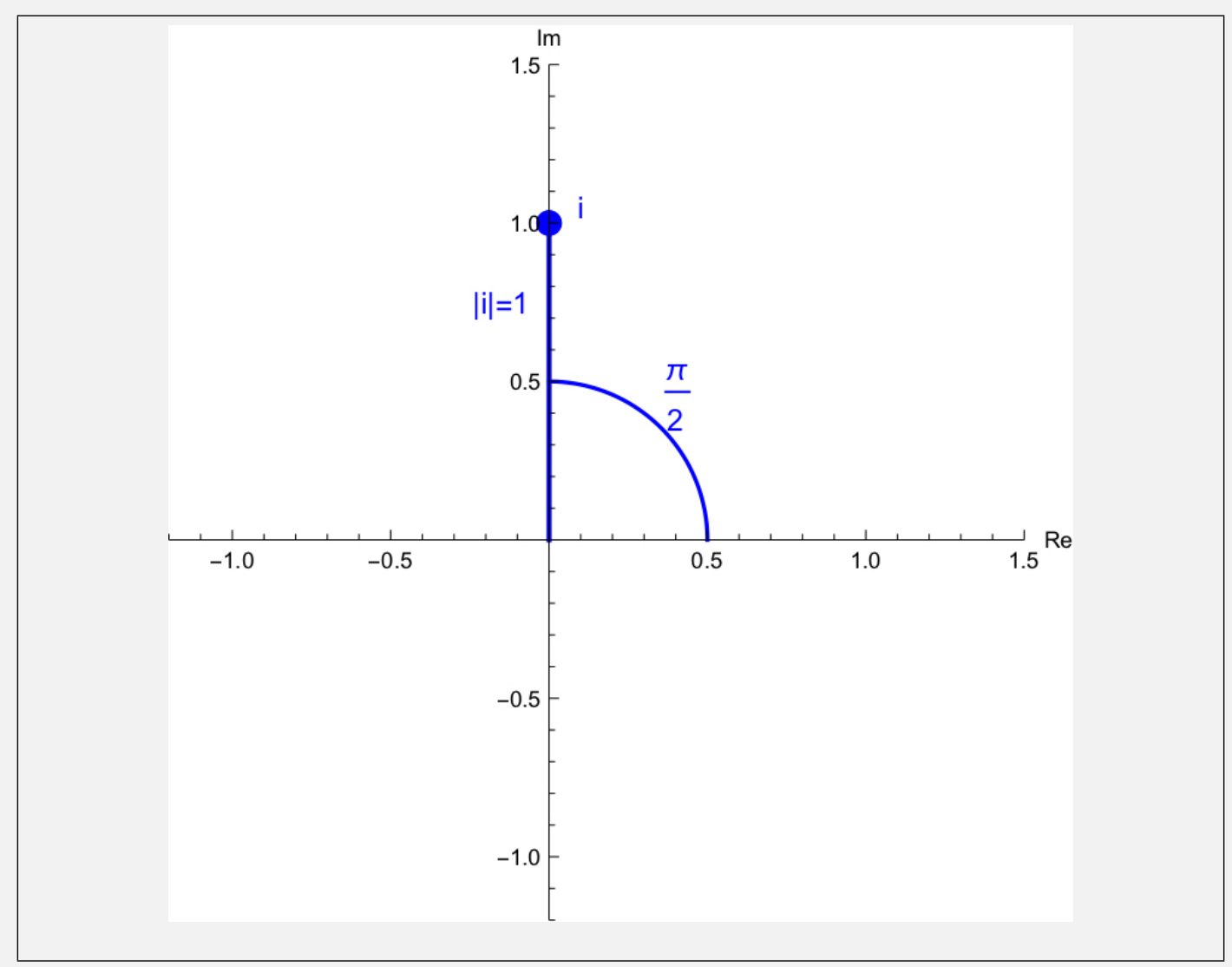

Well, it sits on the imaginary axis, and therefore has principle argument $\frac{\pi}{2}$. Thus:

$$
i=1\left(\cos \left(\frac{\pi}{2}\right)+i \sin \left(\frac{\pi}{2}\right)\right)
$$

This means that multiplying a complex number $z=|z|(\cos \alpha+i \sin \alpha)$ by $i$ can be written as:

$$
z i=|z|\left(\cos \left(\alpha+\frac{\pi}{2}\right)+i \sin \left(\alpha+\frac{\pi}{2}\right)\right)
$$

ie multiplication by $i$ is equivalent to rotation by $\frac{\pi}{2}$ in the complex plane...which is one of the ways that we first came across $i$. 


\subsection{Taking powers of complex numbers}

Now we know how easy it is to multiply together two complex numbers when they are written in modulus-argument form. How about squaring a complex number. This is just a special case of multiplication, where the number is being multiplied by itself. Let's start with complex numbers of modulus 1, and adapt to the more general case later. Take the above expression for $z w$ and make $w$ be the same as $z$. ie. if $z=w=\cos \theta+i \sin \theta$, you get:

$$
z^{2}=\cos (2 \theta)+i \sin (2 \theta)
$$

How about $z^{3}$ ? Well, you just multiply $z^{2}$ by $z$, which yields:

$$
z^{3}=\cos (3 \theta)+i \sin (3 \theta)
$$

In fact, repeated multiplications like this give us a general statement and the name of it is De Moivre's Theorem:

Theorem 1.12.1. De Moivre's Theorem

For any integer $n$ and real number $\theta$,

$(\cos \theta+i \sin \theta)^{n}=\cos (n \theta)+i \sin (n \theta)$

From the picture of multiplication of complex numbers as rotations, de Moivre's theorem should become relatively intuitive. Note that De Moivre's Theorem is for complex numbers with modulus 1 . However, the theorem can be extended to include any complex number $z$ with $|z|=r$ i.e. $z=r(\cos \theta+i \sin \theta)$ :

$$
z^{n}=(r(\cos \theta+i \sin \theta))^{n}=r^{n}(\cos (n \theta)+i \sin (n \theta))
$$

Let's look at the proof of De Moivre's Theorem. 


\section{Proof of theorem 1.12.1}

We use mathematical induction to prove the theorem.

Case $1: n \geq 0$

For any integer $n \geq 0$ let $P(n)$ be the proposition that

$$
(\cos \theta+i \sin \theta)^{n}=\cos (n \theta)+i \sin (n \theta)
$$

Base case: The statement $P(0)$ says that

$$
(\cos \theta+i \sin \theta)^{0}=\cos (0)+i \sin (0)=1
$$

which is true.

Induction Hypothesis (IH): We now assume that $P(m)$ is true for some non-negative integer $m$

$$
(\cos \theta+i \sin \theta)^{m}=\cos (m \theta)+i \sin (m \theta)
$$

Inductive Step: It now remains for us to show that $P(m+1)$ holds true

$$
\begin{aligned}
\cos ((m+1) \theta)+i \sin ((m+1) \theta) & =(\cos \theta+i \sin \theta)^{m+1} \\
& =(\cos \theta+i \sin \theta)^{m}(\cos \theta+i \sin \theta) \\
& =(\cos (m \theta)+i \sin (m \theta))(\cos \theta+i \sin \theta) \\
& =\cos ((m+1) \theta)+i \sin ((m+1) \theta)
\end{aligned}
$$

Theorem 1.11.1

So $P(m+1)$ holds true. By the principle of mathematical induction $P(n)$ is true for all non-negative integers $n$. 
Case 2: $n<0$

Let $n=-k$ where $n$ is any negative integer, so $k$ is a positive integer.

$$
\begin{aligned}
(\cos \theta+i \sin \theta)^{n} & =(\cos \theta+i \sin \theta)^{n} \\
& =(\cos \theta+i \sin \theta)^{-k} \\
& =\frac{1}{(\cos \theta+i \sin \theta)^{k}} \\
& =\frac{1}{\cos (k \theta)+i \sin (k \theta)} \quad \text { Case } \mathbf{1} \\
& =\frac{1}{\cos (k \theta)+i \sin (k \theta)} \times \frac{\cos (k \theta)-i \sin (k \theta)}{\cos (k \theta)-i \sin (k \theta)} \\
& =\frac{\cos (k \theta)-i \sin (k \theta)}{\cos { }^{2}(k \theta)-i^{2} \sin ^{2}(k \theta)} \\
& =\frac{\cos ^{2}(k \theta)-i \sin (k \theta)}{\cos ^{2}(k \theta)+\sin ^{2}(k \theta)} \\
& =\cos (k \theta)-i \sin (k \theta) \\
& =\cos (-n \theta)-i \sin (-n \theta) \\
& =\cos (n \theta)+i \sin (n \theta)
\end{aligned}
$$

We have now proved that $(\cos \theta+i \sin \theta)^{n}=(\cos \theta+i \sin \theta)^{n}$ for any integer $n$. 


\section{Worked Examples 1.12.1.}

(a) Use De Moivre's Theorem to write $(1+i)^{20}$ in a cartesian from.

(b) Write $(\sqrt{3}+i)^{3}(1+\sqrt{3} i)^{5}$ in a modulus-argument form.

(c) Write $\frac{(1+i)^{10}}{1+\sqrt{3} i}$ in a modulus-argument form.

\section{Solutions to worked examples 1.12.1:}

(a) We first need to write this number in modulus argument as that is how de Moivre's theorem is stated. We can write:

$$
(1+i)=\sqrt{2}\left(\cos \frac{\pi}{4}+i \sin \frac{\pi}{4}\right)
$$

and therefore:

$$
\begin{aligned}
(1+i)^{20} & =\left(\sqrt{2}\left(\cos \frac{\pi}{4}+i \sin \frac{\pi}{4}\right)\right)^{20}=\sqrt{2}^{20}\left(\cos \frac{20 \pi}{4}+i \sin \frac{20 \pi}{4}\right) \\
& =2^{10}(\cos 5 \pi+i \sin 5 \pi)
\end{aligned}
$$

But we know that we can always subtract any integer multiple of $2 \pi$ from an argument and it will still be the same complex number. Because the angle here is $5 \pi$ if we subtract 2 lots of $2 \pi$ we will end up with $\pi$ which is a principle argument, so:

$$
(1+i)^{20}=2^{10}(\cos \pi+i \sin \pi)=2^{10}(-1+0 i)=-2^{10}
$$

(b) We will do the same thing for the next example, but without the explanatory steps. Go through and make sure that you can see what is happening here:

$$
\begin{aligned}
(\sqrt{3}+i)^{3}(1+\sqrt{3} i)^{5} & =\left(2 ( \operatorname { c o s } ( \frac { \pi } { 6 } + i \operatorname { s i n } \frac { \pi } { 6 } ) ) ^ { 3 } \left(\left(2\left(\cos \frac{\pi}{3}+i \sin \frac{\pi}{3}\right)\right)^{5}\right.\right. \\
& =2^{3}\left(\cos \frac{3 \pi}{6}+i \sin \frac{3 \pi}{6}\right) 2^{5}\left(\cos \frac{5 \pi}{3}+i \sin \frac{5 \pi}{3}\right) \\
& =2^{3} 2^{5}\left(\cos \left(\frac{3 \pi}{6}+\frac{10 \pi}{6}\right)+i \sin \left(\frac{3 \pi}{6}+\frac{10 \pi}{6}\right)\right. \\
& =2^{8}\left(\cos \frac{13 \pi}{6}+i \sin \frac{13 \pi}{6}\right) \\
& =2^{8}\left(\cos \frac{\pi}{6}+i \sin \frac{\pi}{6}\right) \\
& =2^{8}\left(\frac{\sqrt{3}}{2}+\frac{i}{2}\right)=2^{7}(\sqrt{3}+i)
\end{aligned}
$$

(c) And again, make sure that you can follow all steps:

$$
\begin{aligned}
\frac{(1+i)^{10}}{1+\sqrt{3} i} & =(1+i)^{10}(1+\sqrt{3} i)^{-1} \\
& =\left(\sqrt{2}\left(\cos \frac{\pi}{4}+i \sin \frac{\pi}{4}\right)\right)^{10}\left(2\left(\cos \frac{\pi}{3}+i \sin \frac{\pi}{3}\right)\right)^{-1} \\
& =\frac{2^{5}}{2}\left(\cos \frac{10 \pi}{4}+i \sin \frac{10 \pi}{4}\right)\left(\cos \left(-\frac{\pi}{3}\right)+i \sin \left(-\frac{\pi}{3}\right)\right) \\
& =2^{4}\left(\cos \left(\frac{10 \pi}{4}-\frac{p i}{3}\right)+i \cos \left(\frac{10 \pi}{4}-\frac{p i}{3}\right)\right) \\
& =2^{4}\left(\cos \left(\frac{13 \pi}{6}\right)+i \cos \left(\frac{13 \pi}{6}\right)\right) \\
& =16\left(\sqrt{3} 2+\frac{i}{2}\right)=8(\sqrt{3}+i)
\end{aligned}
$$




\section{Check your understanding 1.12.1.}

(a) Write the following complex numbers in a cartesian form:

(i) $(1-\sqrt{3} i)^{5}$

(ii) $(2 \sqrt{3}+2 i)^{5}$

(iii) $(1-i)^{8}$

(iv) $(1-\sqrt{3} i)^{5}(\sqrt{3}+i)^{2}$

(v) $\frac{(1-\sqrt{3} i)^{5}}{(1-i)^{6}}$

(b) By considering the expansion of $(1+i)^{10}$, show that $\left(\begin{array}{c}10 \\ 1\end{array}\right)-\left(\begin{array}{c}10 \\ 3\end{array}\right)+\left(\begin{array}{c}10 \\ 5\end{array}\right)-\left(\begin{array}{c}10 \\ 7\end{array}\right)+\left(\begin{array}{c}10 \\ 9\end{array}\right)=32$

(c) Let $z=\cos \theta+i \sin \theta$.

(i) Show that $2 \cos \theta=z+\frac{1}{z}$.

(ii) Show that $2 \cos (n \theta)=z^{n}+\frac{1}{z^{n}}$.

(d) Find the exact value of $\frac{1}{(\sqrt{3}+i)^{6}}$, clearly showing your working.

(e) (i) Express $\frac{\sqrt{3}}{2}-\frac{1}{2} i$ in the modulus-argument form.

(ii) Hence show that $\left(\frac{\sqrt{3}}{2}-\frac{1}{2} i\right)^{9}=c i$ where $c$ is a real number to be found.

(iii) Find one pair of possible values of positive integers $m$ and $n$ such that

$$
\left(\frac{\sqrt{3}}{2}-\frac{1}{2} i\right)^{m}=\left(\frac{\sqrt{2}}{2}+\frac{\sqrt{2}}{2} i\right)^{n}
$$

\section{Section 2.12 Checklist}

$\square$ I know how to multiply two complex numbers in a modulus-argument form.

$\square$ I can prove Theorems 1.11.1 and 1.12.1.

$\square$ I know how to use De Moivre's Theorem to take powers of complex numbers in a modulusargument form. 


\subsection{Functions of a complex variable}

We have already hinted at one function of a complex number, but we haven't really thought about it very deeply. So far in general we have only looked at real-valued functions of a real variable. $f: \mathbb{R} \rightarrow \mathbb{R}$.

We often use a graphical way of describing such a function using the curve $y=f(x)$ in the $(x, y)$ plane, which says that for each ordered pair $(x, y)$ such that $y=f(x)$ there is a point. In fact this doesn't need to give a curve, as it could just be a discrete set of points, but generally it will be a curve.

Can we do the same thing for a function of the form $f: \mathbb{C} \rightarrow \mathbb{C}$ ? We would need to find points $(z, w)$ such that $w=f(z)$ but both $z$ and $w$ are complex numbers which can only be denoted by two real numbers. ie. $z=a+b i$ and $w=c+d i$. So really we need four real numbers $(a, b, c, d)$, such that $c+d i=f(a+b i)$. So we need four axes? Well, that's not going to work with our simple two dimensional paper!

Instead of this, we write down two versions of the complex plane, one for $z$ and one for $w$. We then draw on some set of points on the $z$ plane and ask where those points go to under the map. The points we chose may be discrete points, or they may be a curve.

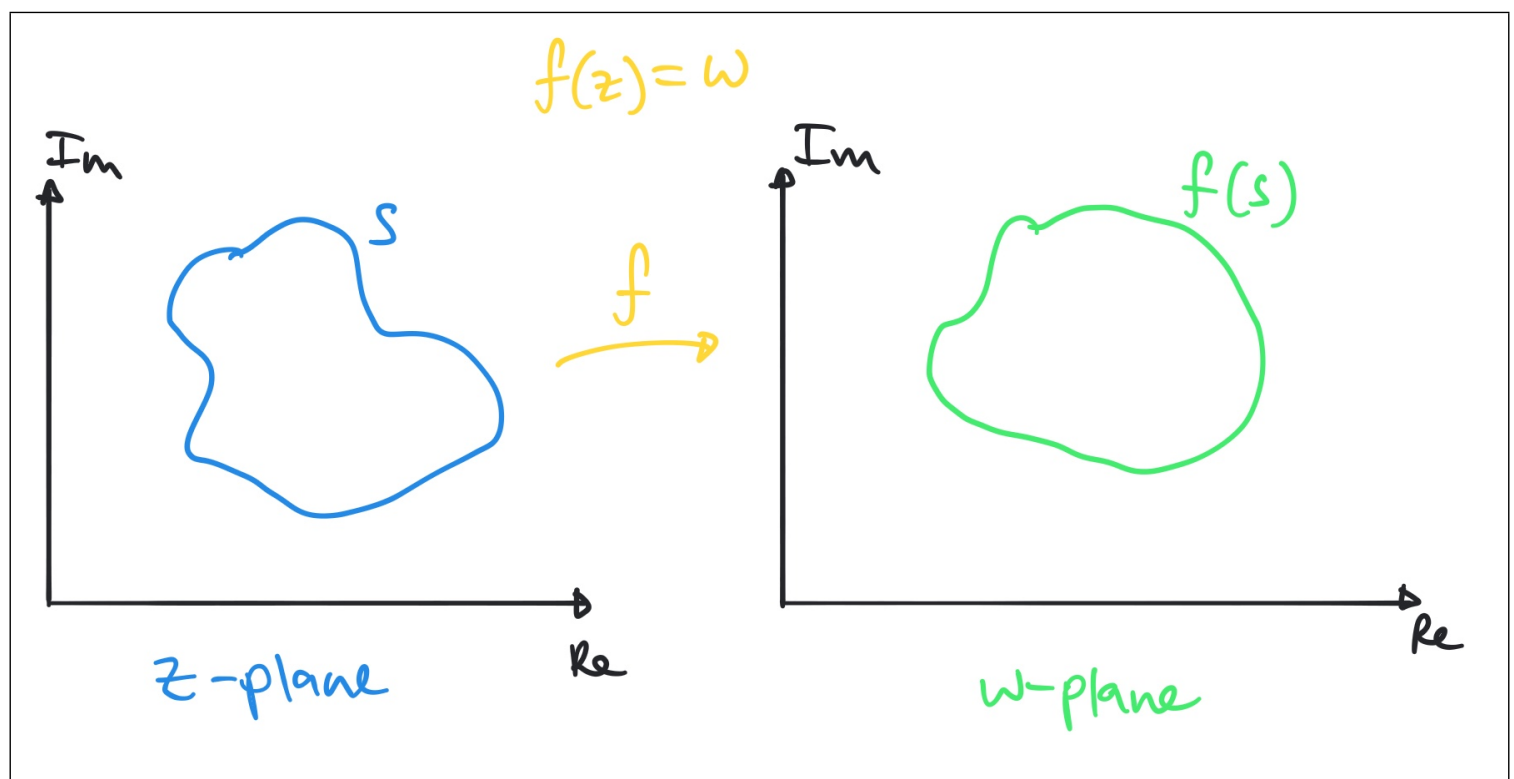

Figure 1.24: The mapping of a set $S$ to $f(S)$, the image of set $S$ under the mapping $f$.

We already saw one example of this in the section where we were asking about the multiplicative inverse of a complex number where $f(z)=1 / z$. We will see another example in a little bit. 


\subsection{The Complex Exponential Function}

Here we are going to get onto slightly more advanced functions of complex numbers. Up to now we can perform all the basic arithmetic operations on them. and can describe them in a number of ways. We can also take powers of them. We are going to go one step further and ask if we can exponentiate them.

It's pretty clear what taking a power of a complex number means once you understand multi-

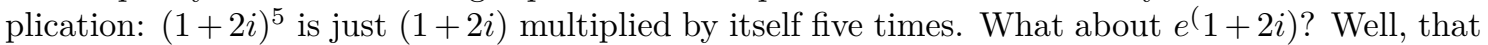
means taking the number $e$ and multiplying it by itself $(1+2 i)$ times....erm, ok! That doesn't make much sense, does it?

Well, we are going to see that we can define complex exponentiation without needing to understand what it means to multiply something by itself that many times.

The way we are going to define this is to go back to our Maclaurin polynomial definition and just assume that we can extend this to complex numbers. That seems pretty risky, but it will reap some rich rewards. In fact one has to be really careful about when and how you can do this, and we aren't able to go that far here, but if you want to do everything really correctly you do have to worry about such things.

We need to remember three things. Recall the MacLaurin polynomial for $e^{x}$ where $x \in \mathbb{R}$ :

$$
e^{x}=1+x+\frac{x^{2}}{2}+\frac{x^{3}}{3 !}+\frac{x^{4}}{4 !}+\frac{x^{5}}{5 !}+\ldots \ldots+\frac{x^{n}}{n !}
$$

and for $\cos x$ is

$$
\cos x=1-\frac{x^{2}}{2 !}+\frac{x^{4}}{4 !}-\frac{x^{6}}{6 !}+\frac{x^{2 n}}{(2 n) !}
$$

and for $\sin x$ is

$$
\sin x=x-\frac{x^{3}}{3 !}+\frac{x^{5}}{5 !}-\frac{x^{7}}{7 !}+\frac{x^{2 n-1}}{(2 n-1) !}
$$

In order to take the Maclaurin polynomial for $e^{i y}$ we are going to do nothing more than replacing $x$ with $i y$ where $y \in \mathbb{R}$ :

$$
\begin{aligned}
e^{i y} & =1+i y+\frac{(i y)^{2}}{2 !}+\frac{(i y)^{3}}{3 !}+\frac{(i y)^{4}}{4 !}+\ldots \ldots+\frac{(i y)^{n}}{n !} \\
& =1+i y-\frac{y^{2}}{2 !}-\frac{i y^{3}}{3 !}+\frac{y^{4}}{4 !}+\frac{i y^{5}}{5 !}+\frac{i y^{6}}{6 !}+\frac{i y^{7}}{7 !}+\ldots
\end{aligned}
$$

ok, let's do a bit of rearranging now...

$$
e^{i y}=\left(1-\frac{y^{2}}{2 !}+\frac{y^{4}}{4 !}-\frac{y^{6}}{6 !}+\ldots\right)+i\left(y-\frac{y^{3}}{3 !}+\frac{y^{5}}{5 !}-\frac{y^{7}}{7 !}+\ldots\right)
$$

But hang on a moment, we should recognise this from the Maclaurin polynomials of $\cos x$ and $\sin x$ that we wrote above. Remember that $y$ is a real number, so

$$
e^{i y}=\cos y+i \sin y
$$

But this is truly remarkable! Let's step back and see what we have just discovered. Apparently the exponential of an imaginary number (not fully complex yet) is taken by calculating the basic trig functions on the imaginary part of that number. You definitely couldn't plug $e^{3 i}$ into a basic calculator (ok, you could on a more complicated one), and so had I asked you what $e^{3 i}$ was you wouldn't be able to point to where it is in the complex plane. However, you can definitely calculate $\cos 3$ and $\sin 3$, and these are just the real and imaginary parts of $e^{3 i}$ so you can now point out where it is in the complex plane....go on, do it. 
What we have just found is that there is an intimate connection between expoential functions and trigonometric functions and that connection is something to do with complex numbers. You never would have expected them to have anything to do with each other. $e$ shows up when thinking about differentiation and integration. Trig functions show up when thinking about circles. This is really beautiful and it was all made possible by Maclaurin polynomials.

$\mathrm{Ok}$, so we can actually go a bit further than this. Here we have only looked at the exponential of an imaginary number. What about a fully complex number (with a real and imaginary part.

Well, in fact for both of these, because we haven't been completely sure that we can use the Maclaurin polynomial of a real number on an imaginary number, we are just going to make these definitions and then see what the consequences are:

Definition 1.14.1. Euler's formula and the complex exponential function

For $x, y \in \mathbb{R}$ we define:

(a) $e^{i y}=\cos y+i \sin y($ Euler's formula)

(b) $e^{z}=e^{x+i y}=e^{x} e^{i y}=e^{x}(\cos y+i \sin y)$

A special case of Euler's formula (when $y=\pi$ ) is called Euler's Identity and is considered by many as the most beautiful equations in mathematics:

$$
e^{i \pi}+1=0
$$

Many people think of Euler's formula as the most beautiful formula in all of mathematics as it involves five numbers from very different spheres of the number realm. Why should $e$ and $\pi$ and $i$ and integer values 0 and 1 have anything to do with each other? In fact when you understand the formula it is really obvious, but without understand complex numbers it makes no sense at all.

This relationship between exponential and trig functions allows us to write the modulus argument form in an even simpler way than before:

For a non-zero complex number, the modulus-argument form i.e. $z=|z|(\cos \theta+i \sin \theta)$ can now be conveniently written as:

$$
z=|z| e^{i \theta}
$$

We need to get a bit of a feel for what the exponential of a complex number will be. Here is a useful exercise:

1. Take a sheet of paper and draw a line down the middle horizontally.

2. In the top half draw a copy of the complex plane, with both real and imaginary values ranging from -2 to 2 .

3. Randomly draw some points in the plane in positions that you can estimate the actual value of the complex number (ie. one of them might be at 1.5-0.5i. Draw some along the axes, and some not on the axes. Maybe draw 10 points.

4. For each of these calculate the exponential using the formula above.

5. Now draw another copy of the complex plane in the bottom half of the page.

6. Plot the positions of the exponentials of the complex numbers that you had in the top half - ie. the answers you found when you calculated the exponential of the first points.

7. See if you can see any pattern that is emerging. Do you see any periodicity? Do you see what makes the exponential smaller or larger, or in different positions in the complex plane? 
In the following we do this not for a series of discrete points, but for a series of lines. See if you can figure out which red lines on the left are mapped to which red lines on the right and blue lines to blue lines under the complex exponential function.

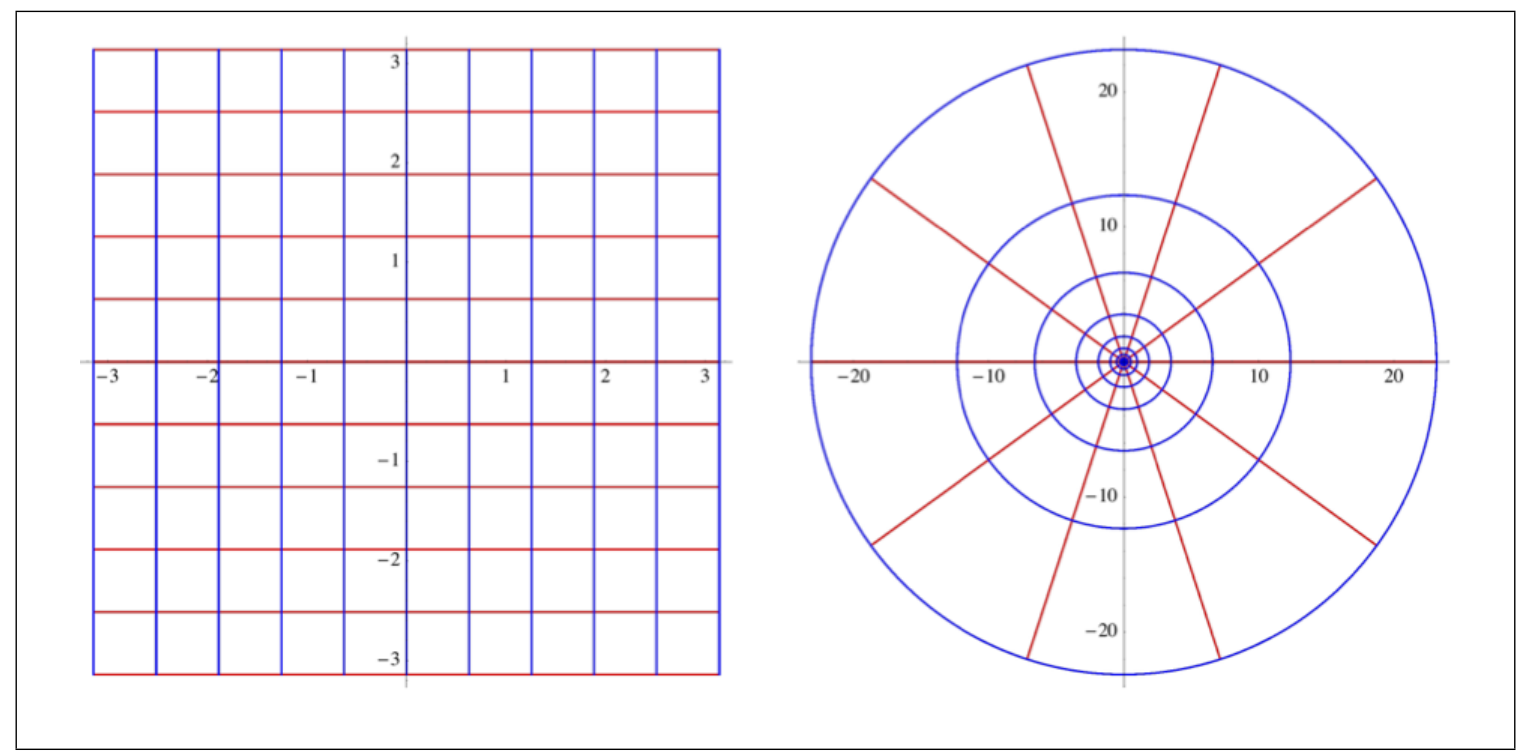

Figure 1.25: The complex plane mapped under the exponential function

One really important point to note here is that infinite lines get mapped to circles. This you might have guessed by the way the exponential function is really trigonometric in disguise, and is thus periodic. This means that it's not invertible. It's many to one (just as for cos and sin). 
As we have done before, once we write down a definition we can explore its properties. We will list these but each of them can be proved.

Property 1.14.1. Properties of the complex exponential function:

If $z_{1}, z_{2}$ and $z=x+i y$ are complex numbers, and $n, k \in \mathbb{Z}$ then,

(a) $e^{z_{1}} e^{z_{2}}=e^{z_{1}+z_{2}}$

(b) $e^{z_{1}-z_{2}}=\frac{e^{z_{1}}}{e^{z_{2}}}$

(c) $\left(e^{z}\right)^{n}=e^{z n}$

(d) $\arg \left(e^{z}\right)=y+2 \pi n$

(e) $\left|e^{z}\right|=e^{x}$

(f) If $e^{z}=1$ then $z=2 \pi k i$

(g) If $e^{z_{1}}=e^{z_{2}}$ then $z_{1}-z_{2}=2 k \pi i$

(h) $e^{z+2 \pi i n}=e^{z}$

Remark: Property 1.14.1(h) tells us that $f(z)=e^{z}$ is a periodic function with period $2 \pi i$. This implies that $f(z)=e^{z}$ has no inverse function, unless we restrict the domain of $f(z)$, in this course, we will not define the complex logarithm function, you'll see the function in MAM3000W if you take complex analysis.

We will prove properties 1.14.1(a) and 1.14.1(e) leave the rest for homework exercises.

Proof of property 1.14.1(a): Let $z_{1}=x_{1}+i y_{1}$ and $z_{2}=x_{2}+i y_{2}$ where $x_{1}, x_{2}, y_{1}, y_{2} \in \mathbb{R}$.

$$
\begin{aligned}
e^{z_{1}} e^{z_{2}} & =e^{x_{1}+i y_{1}} e^{x_{2}+i y_{2}} \\
& =e^{x_{1}} e^{i y_{1}} e^{x_{2}} e^{i y_{2}} \\
& =e^{x_{1}}\left(\cos y_{1}+i \sin y_{1}\right) e^{x_{2}}\left(\cos y_{2}+i \sin y_{2}\right) \\
& =e^{x_{1}} e^{x_{2}}\left(\cos \left(y_{1}+y_{2}\right)+i \sin \left(y_{1}+y_{2}\right)\right) \\
& =e^{x_{1}} e^{x_{2}} e^{i\left(y_{1}+y_{2}\right)} \\
& =e^{x_{1}+x_{2}} e^{i\left(y_{1}+y_{2}\right)} \\
& =e^{\left(x_{1}+x_{2}\right)+i\left(y_{1}+y_{2}\right)} \\
& =e^{\left(x_{1}+i y_{1}\right)+\left(x_{2}+i y_{2}\right)} \\
& =e^{z_{1}+z_{2}}
\end{aligned}
$$

Definition 1.14.1(b)

Definition 1.14.1(a)

Theorem 1.11.1

Definition 1.14.1(a)

Exponential laws for $\mathbb{R}$

Definition 1.14.1(b) 
Proof of property 1.14.1(e): Let $z=x+i y$ where $x, y \in \mathbb{R}$.

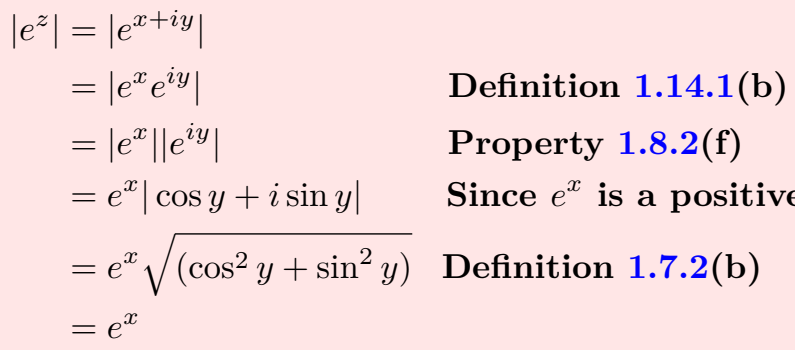

\section{Worked Examples 1.14.1.}

(a) Solve for $z \in \mathbb{C}$ and plot all the solutions on an Argand diagram:
(i) $e^{z}=\sqrt{3}+i$
(ii) $e^{i z}=1+\sqrt{3} i$
(iii) $e^{z}=|1+\sqrt{3} i|$

(b) Sketch the image of set $S$ under the mapping $f$ if:

$$
f(z)=e^{z} \text { and } S=\left\{z \in \mathbb{C} \mid 1 \leq \operatorname{Re}(z)<2 \quad,-\frac{\pi}{6}<\operatorname{Im}(z) \leq \frac{\pi}{3}\right\}
$$

\section{Solutions to worked examples 1.14.1:}

(a) (i) Let $z=x+i y$, where $x, y \in \mathbb{R}$, ie. $e^{z}=e^{x+i y}=e^{x} e^{i y}$. Then write the right hand side in exponential form:

$$
e^{x} e^{i y}=\sqrt{3}+i=2 e^{i\left(\frac{\pi}{6}+2 \pi k\right)}
$$

where $k \in \mathbb{Z}$. Now we equate the moduli and argument of both sides to get:

$$
e^{x}=2 \Longrightarrow x=\ln 2
$$

and

$$
y=\frac{\pi}{6}+2 \pi k
$$

which means that

$$
z=x+i y=\ln 2+i\left(\frac{\pi}{6}+2 \pi k\right), \quad k \in \mathbb{Z}
$$

(All solutions will be plotted on the same diagram after the last example.

(ii) The same process here. Let $z=x+i y$ :

$$
\begin{aligned}
e^{i z} & =e^{i}(x+i y)=e^{-y} e^{i x}=1+\sqrt{3} i \Longrightarrow \\
e^{-y} e^{i x} & =\sqrt{2} e^{i\left(\frac{\pi}{3}+2 \pi k\right)}, k \in \mathbb{Z} \\
& \Longrightarrow e^{-y}=2, \quad x=\frac{\pi}{3}+2 \pi k \\
& \Longrightarrow z=\frac{\pi}{3}+2 \pi k-i \ln 2
\end{aligned}
$$

(iii) And again. Let $z=x+i y$ :

$$
\begin{aligned}
e^{x+i y} & =e^{x} e^{i y}=|1+\sqrt{3} i|=2 e^{i 2 \pi k}, \quad k \in \mathbb{Z} \\
& \Longrightarrow \quad x=\ln 2, y=2 \pi k \\
& \Longrightarrow \quad z=\ln 2+2 \pi k
\end{aligned}
$$




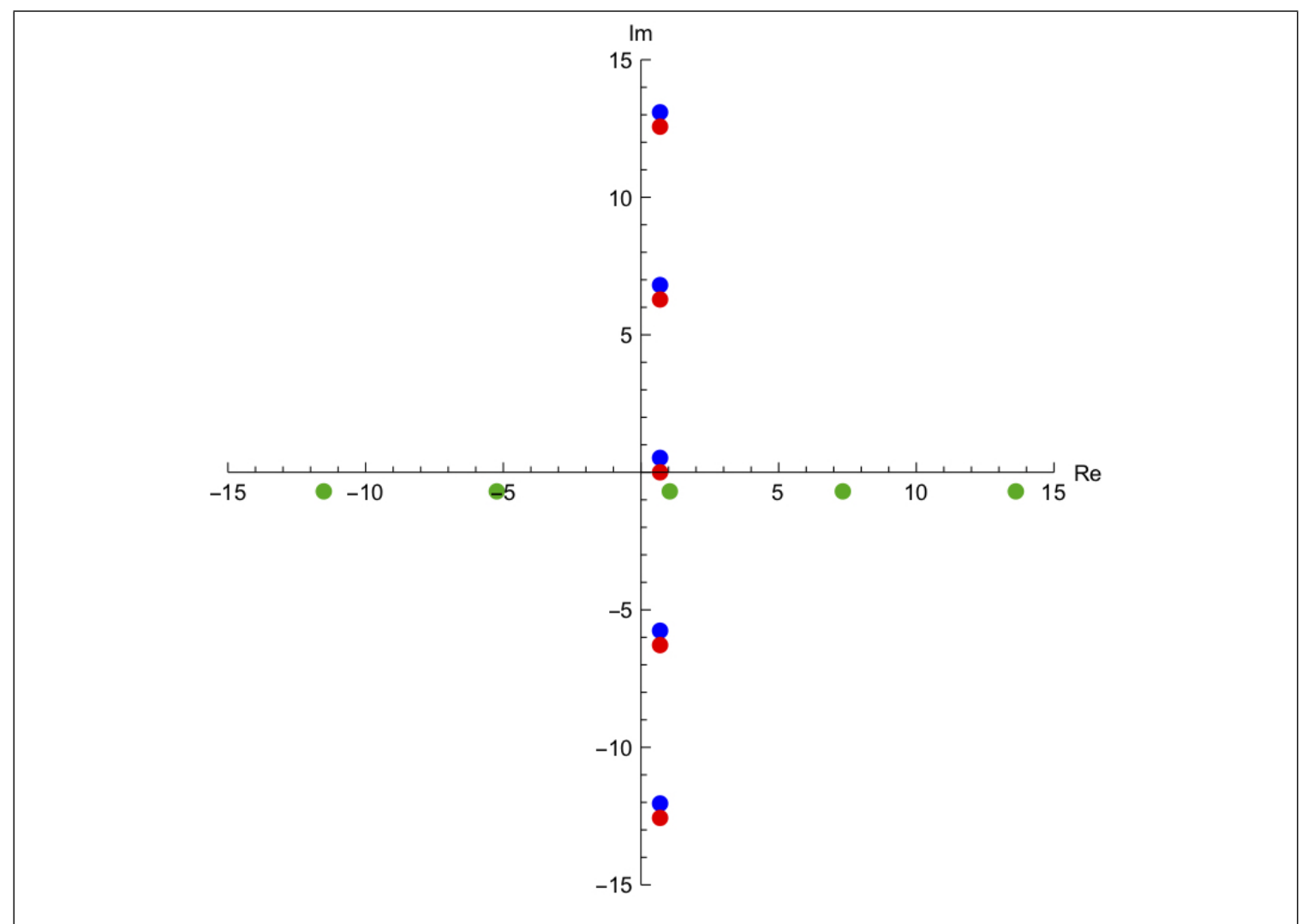

Figure 1.26: (i): Blue, (ii): Green, (iii): Red. These are a subset of the infinite families of solutions.

(b) We are here given a set $S$ which is some region in the complex plane, and we are asked what happens to this set of numbers when we apply the exponential function to them. Let's start by drawing the original set:

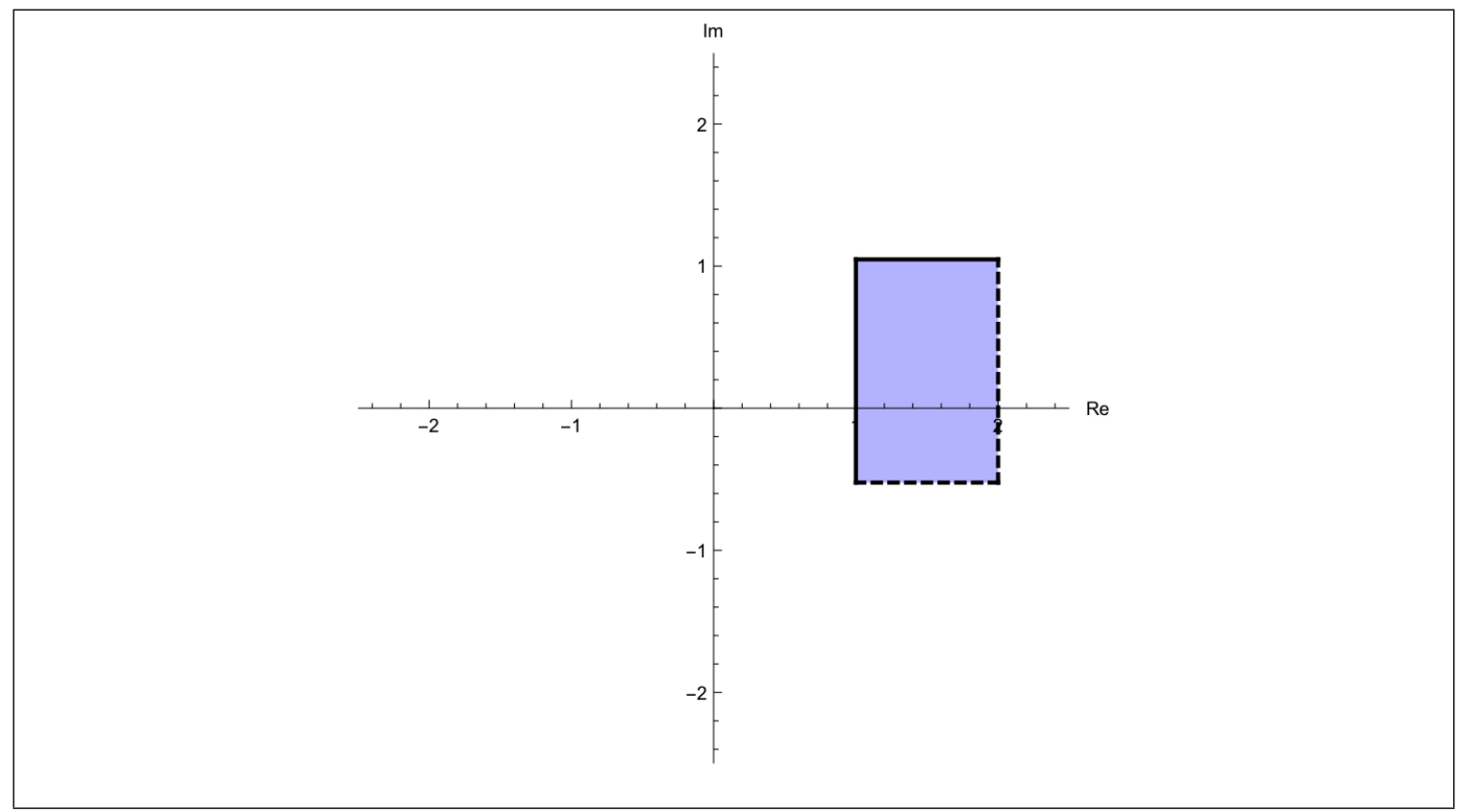

Figure 1.27: The question is what do the points in this region get mapped to under the exponential function?

We can start with something slightly simpler than the whole region. Let's just take the left, vertical side of the region. This set could be denoted as 


$$
\left\{z \in \mathbb{C} \mid \operatorname{Re}(z)=1,-\frac{1}{\sqrt{3}}<\operatorname{Im}(z) \leq \sqrt{3}\right\}
$$

ie. these are numbers of the form $z=1+i b$ where $\left.-\frac{1}{\sqrt{3}}<b \leq \sqrt{3}\right\}$. What happens to these when we exponentiate them? Well, they become:

$$
e^{1+i b}=e^{1} e^{i b}
$$

These are numbers with modulus $e$ and argument $b$ where $\left.-\frac{1}{\sqrt{3}}<b \leq \sqrt{3}\right\}$. This corresponds to an arc of radius $e$ sweeping out the range of angles given by $b$. It looks like:

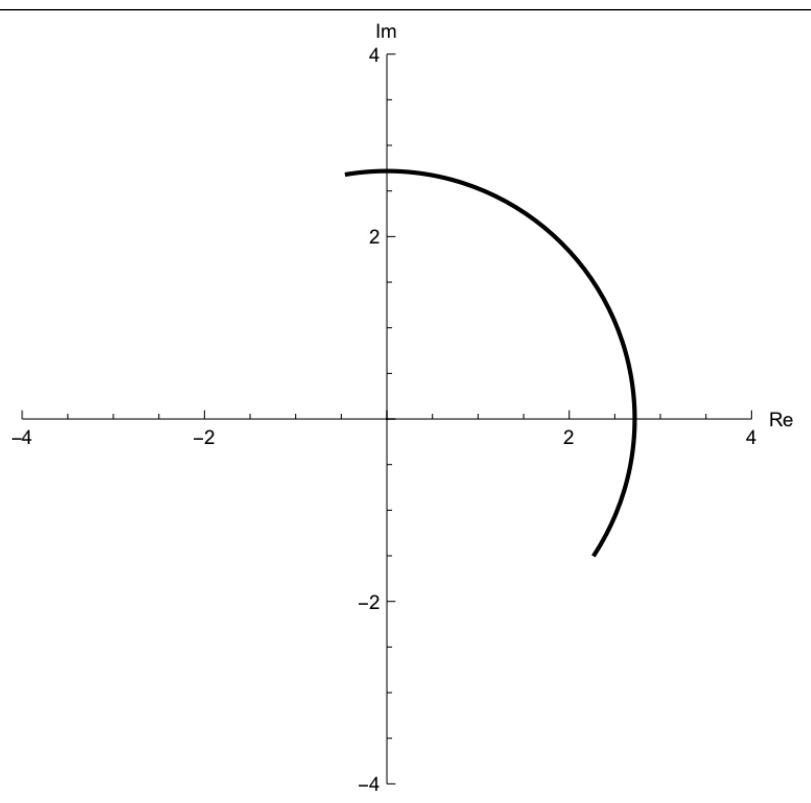

Figure 1.28: The left-most vertical line gets mapped to an arc of radius $e$.

We could do exactly the same thing with the outer vertical line and this would give the same thing, except it would have radius $e^{2}$ :

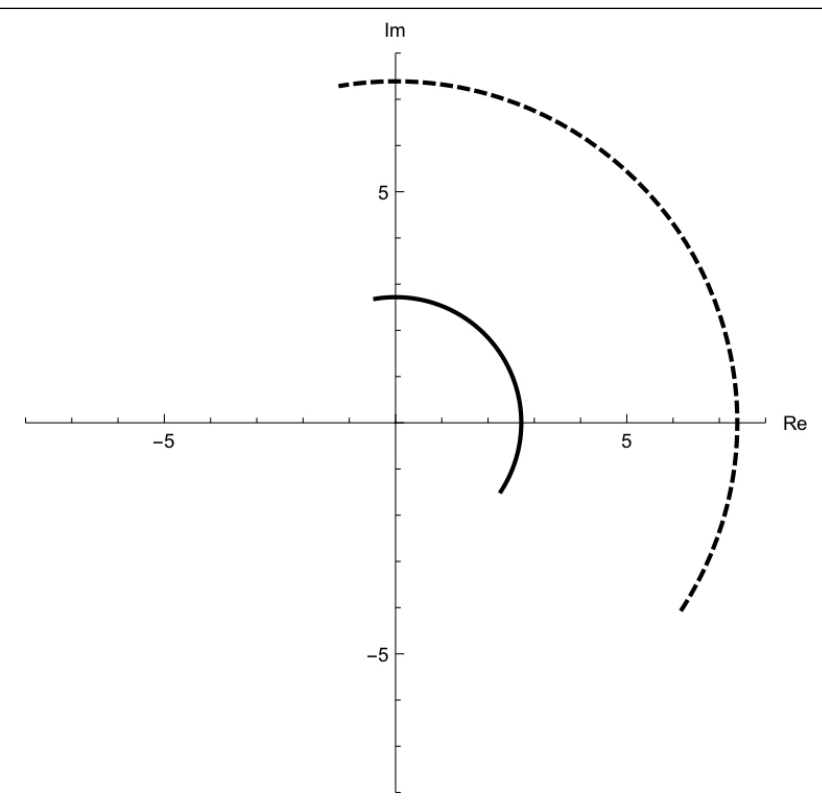

Figure 1.29: The right-most vertical line (which is not actually included in the region) gets mapped to an arc of radius $e^{2}$. 
How about the top sloping line in the original region? This is the set of points:

$$
\{z \in \mathbb{C} \mid 1 \leq \operatorname{Re}(z)<2, \operatorname{Im}(z)=\sqrt{3}\}
$$

That is:

$$
z=a+i \sqrt{3}
$$

where $1 \leq a<2$. When we exponentiate this we get:

$$
e^{z}=e^{a} e^{i \sqrt{3}}=e^{a}(\cos \sqrt{3}+i \sin \sqrt{3})=e^{a}(\cos \sqrt{3}+i \sin \sqrt{3})
$$

This is a set of numbers with modulus $e^{a}$ where $1 \leq a<2$ and with constant argument given by $\sqrt{3}$. This gives the new line in the following:

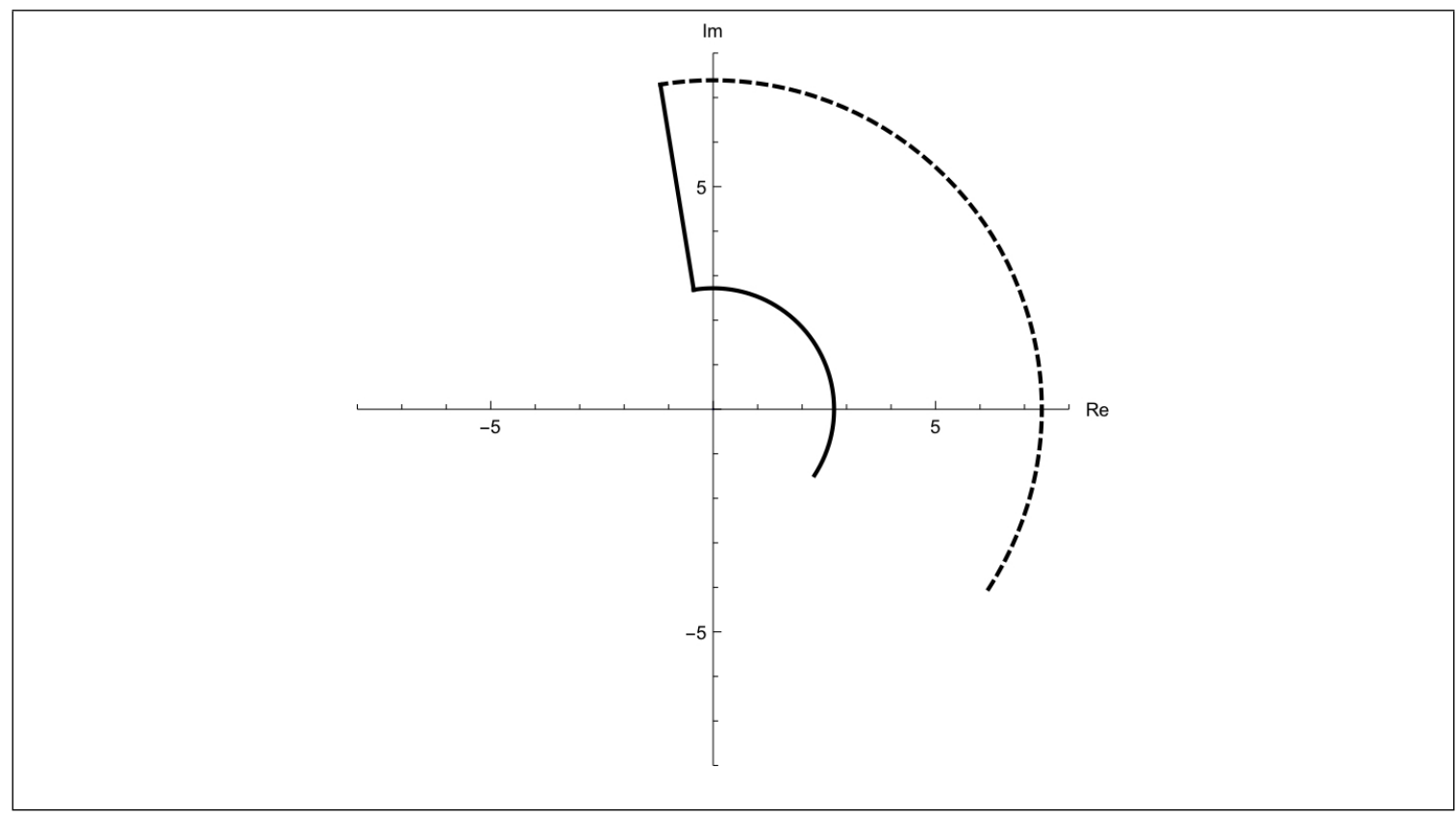

Figure 1.30: The top line gets mapped to the new line here

And finally for the lower horizontal line we do the same thing as above and come to:

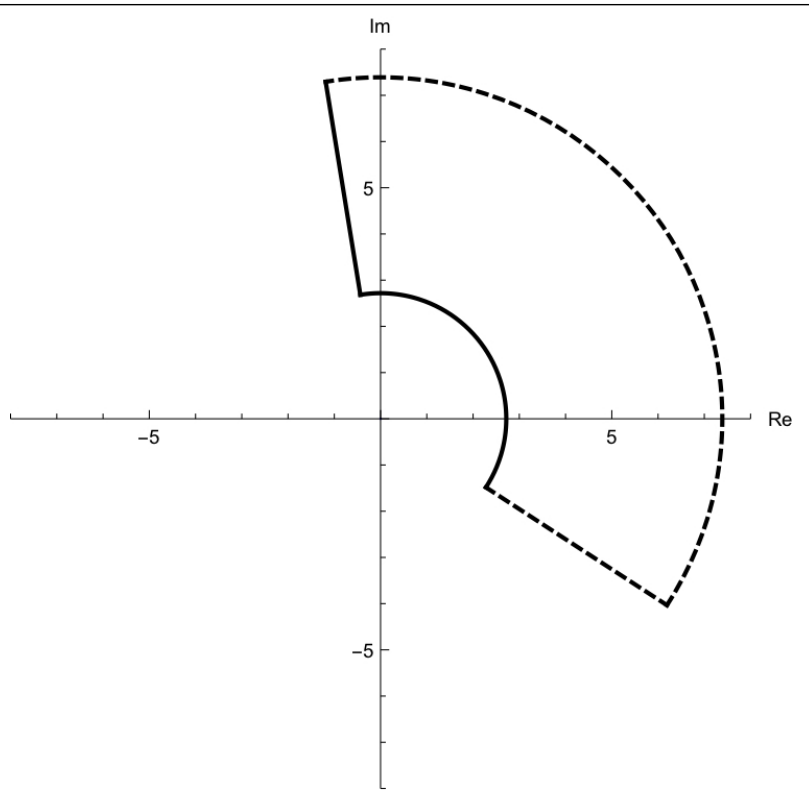

Figure 1.31: All four sides of the region are now mapped. 
Convince yourselves at this stage that in fact any point in the middle of the original set will be mapped to a point inside this new region. Thus we have:

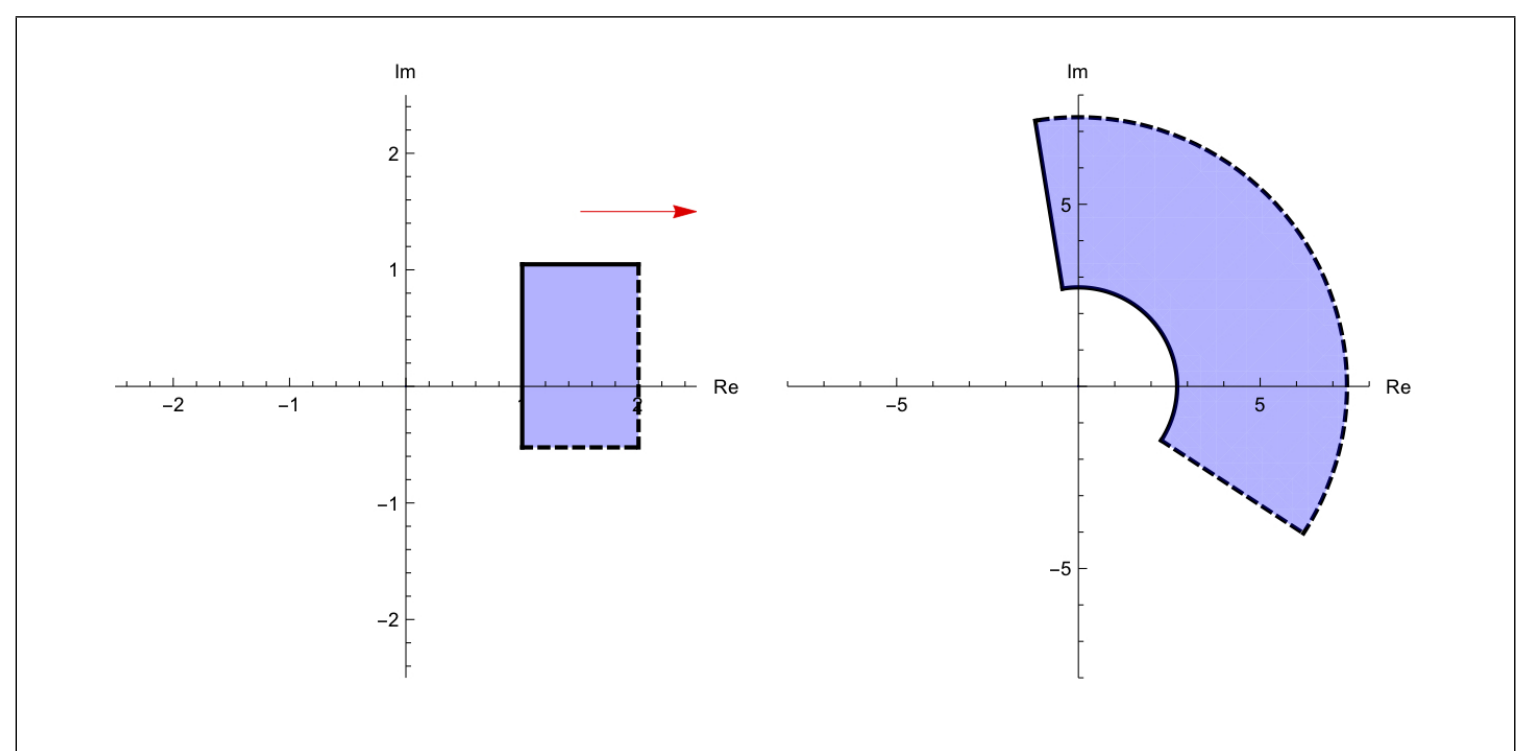

Figure 1.32: and the interior of the region is also mapped. 


\section{Check your understanding 1.14.1.}

(a) Solve for $z \in \mathbb{C}$ and write the solution is a cartesian form. Sketch all the solutions on an Argand diagram if:

(i) $e^{z}=1+i$ (ii) $e^{i z}=1+i$ (iii) $e^{-z}=-\sqrt{3}+i$ (iv) $e^{z}=|1+i|$ (v) $e^{\overline{z+i}}=4 i$ (vi) $\left|e^{z}\right|=|1+i|$ (vii) $e^{|z|}=|1+i|$

\section{Useful facts:}

If $z_{1}$ and $z_{2}$ are complex numbers, then:

1. $z_{1} z_{2}=\left(r_{1} e^{i \theta_{1}}\right)\left(r_{2} e^{i \theta_{2}}\right)=r_{1} r_{2} e^{i\left(\theta_{1}+\theta_{2}\right)}$

2. $\frac{z_{2}}{z_{1}}=\frac{r_{2} e^{i \theta_{2}}}{r_{1} e^{i \theta_{1}}}=\frac{r_{2}}{r_{1}} e^{\theta_{2}-\theta_{1}}$ provided $z_{1} \neq 0$

(b) Prove the following properties of the complex argument:
(i) $\operatorname{Arg}\left(z_{1} z_{2}\right)=\operatorname{Arg}\left(z_{1}\right)+\operatorname{Arg}\left(z_{2}\right)$
(ii) $\operatorname{Arg}\left(z_{1} \overline{z_{2}}\right)=\operatorname{Arg}\left(z_{1}\right)-\operatorname{Arg}\left(z_{2}\right)$

(c) Show that:
(i) $e^{z+\pi i}=-e^{z}$
(ii) $\overline{e^{z}}=e^{\bar{z}}$

(d) If $\alpha$ is an argument of $w$ and $\beta$ is an argument of $z$ (where both $w$ and $z$ are complex numbers, find the argument of $\frac{w^{2} z}{\bar{w}}$ if possible.

(e) If $\operatorname{Arg}\left((a+i)^{3}\right)=\pi$, where $a$ is real and positive, find the exact value of $a$.

(f) Write the following complex numbers in a cartesian form i.e. form $a+b i$

(i) $(\sqrt{3}-i)^{6}$

(ii) $\left(\frac{\sqrt{3}}{2}-\frac{i}{2}\right)^{24}$

(iii) $(-1+i)^{16} \cdot e^{-\frac{i \pi}{2}}$

(iv) $(\sqrt{3}-i)^{5}\left(-\frac{1}{2}+\frac{\sqrt{3}}{2} i\right)^{15}$

(g) If $z=\sqrt{3}+i$ and $w=1+\sqrt{3} i$ find modulus-argument forms for $z w, \frac{z}{w}$, and $\frac{1}{z}$.

(h) Sketch the image of set $S$ under the mapping $f$ if:

$$
f(z)=e^{i z} \text { and } S=\left\{z \in \mathbb{C} \mid \frac{\pi}{6} \leq \operatorname{Re}(z)<\frac{\pi}{3}, 0<\operatorname{Im}(z) \leq 1\right\}
$$


(i) Show that the function $w=e^{z}$ maps the rectangle in Figure 14(a) to the semi-annulus in Figure 14(b)
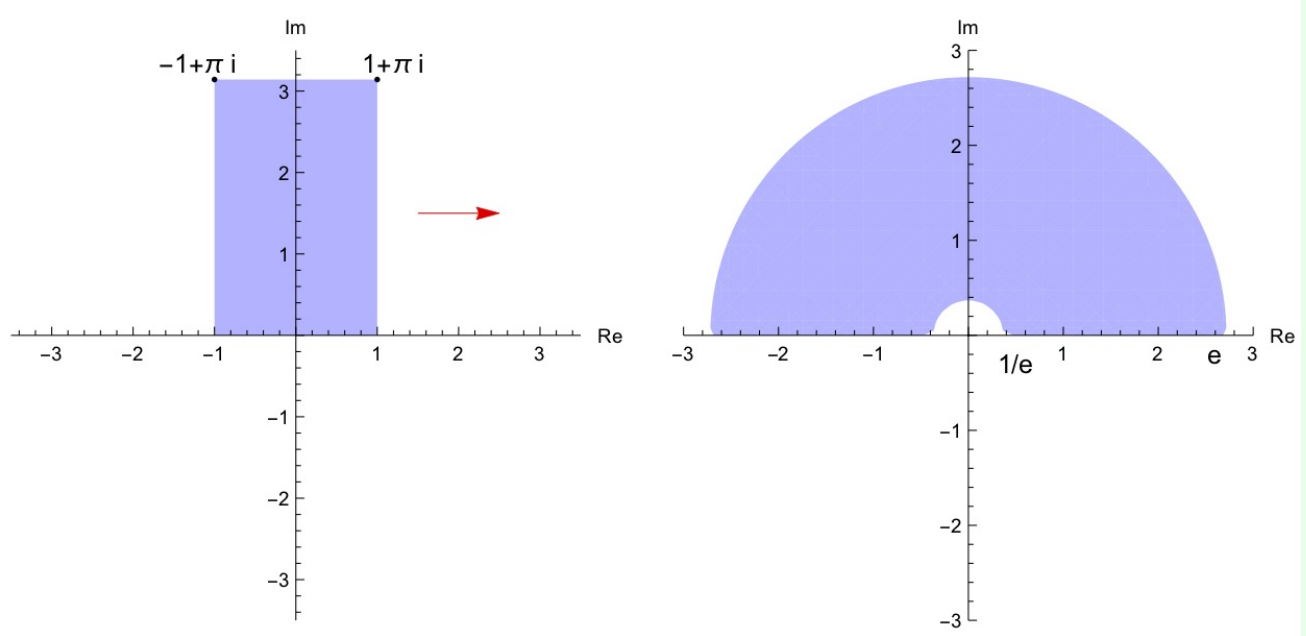

Figure 1.33: The function $e^{z}$ mapping a rectangle to a semi-annulus the plane

(ii) Prove properties 1.14.1.

\section{Section 2.14 Checklist}

$\square$ I know how to write a complex number $z=a+b i$ in a complex exponential form.

$\square$ I know all the three forms of a complex number, and I am comfortable with changing a form of a complex number from one form to another.

$\square$ I can prove all the properties of the complex exponential function.

$\square$ I know that $e^{z}$ is a periodic function that is not one-to-one, and therefore doesn't have an inverse, preventing me from taking a logarithm of a complex number.

$\square$ For a given complex number $w$, I know how to find all the complex numbers $z$ that satisfy the equation $e^{z}=w$.

$\square$ I know how to sketch the solutions of the equation $e^{z}=w$ where $w$ is a given complex number.

$\square$ I know how to map a set of complex numbers under the function $f(z)=e^{z}$. 


\subsection{Complex trigonometric functions}

ok, so now you can calculate where in the complex plane a number like $e^{-2+3 i}$ is by writing this in terms of trig functions:

$$
e^{-2+3 i}=e^{-2}(\cos 3+i \sin 3)
$$

and all the stuff on the right you can calculate very easily on even the simplest of calculators.

How about $\cos (-2+3 i)$. What is this? Where does it sit in the complex plane? Well, you certainly can't type this into a basic calculator. How can we work out trig functions applied to complex numbers? Well, we're going to use some clever footwork and take advantage of what we did in the previous steps. Here it is:

Recall Euler's formula:

$$
e^{i \theta}=\cos \theta+i \sin \theta
$$

Let $\theta=-\theta$ :

$$
e^{-i \theta}=\cos \theta-i \sin \theta
$$

$(1)+(2)$ yields:

$$
2 \cos \theta=e^{i \theta}+e^{-i \theta} \Rightarrow \cos \theta=\frac{e^{i \theta}+e^{-i \theta}}{2}
$$

(1)-(2) yields:

$$
\sin \theta=\frac{e^{i \theta}-e^{-i \theta}}{2 i}
$$

We use the equations above as a motivation for the definitions that follow.

\section{Definition 1.15.1. The complex trigonometric function}

Given any complex number $z$, we define :

$$
\sin z=\frac{e^{i z}-e^{-i z}}{2 i} \text { and } \cos z=\frac{e^{i z}+e^{-i z}}{2}
$$

Remark: One big difference between real and complex trigonometric functions is that real sine and cosine functions are bounded between -1 and 1 , while the complex functions are not bounded if they are defined over the set of all complex numbers (see examples that follow).

So, how could we use this to calculate $\cos (-2+3 i)$ ?

Well, according to the above definition:

$$
\cos (-2+3 i)=\frac{e^{i(-2+3 i)}+e^{-i(-2+3 i)}}{2}=\frac{e^{(-3-2 i)}+e^{(3+2 i)}}{2}
$$

And now we can use the definition of the complex exponential to give us:

$\cos (-2+3 i)=\frac{e^{-3}(\cos (-2)+i \sin (-2))+e^{3}(\cos (2)+i \sin (2))}{2}=\frac{e^{-3} \cos (-2)+e^{3} \cos 2}{2}+i \frac{e^{-3} \sin (-2)+e^{3} \sin 2}{2}$

which can be written as: 


$$
\cos (-2+3 i)=\frac{\cos 2\left(e^{-3}+e^{3}\right)}{2}+i \frac{\sin 2\left(e^{3}-e^{-3}\right)}{2}
$$

and all of this can be calculated on a basic calculator. It looked like quite a lot of work, but the steps are all pretty simple. This means that you can now calculate the sin and cos of any complex number you want and know where it sits in the complex plane.

This is not going to be the only time you will see "trigonometric functions" related to exponential functions. You'll come across (if you haven't already) hyperbolic functions whose definitions resemble those of the complex trigonometric functions. The most common hyperbolic functions are the hyperbolic sine, denoted sinh, and the hyperbolic cosine, denoted cosh, defined by:

$$
\sinh x=\frac{e^{x}-e^{-x}}{2}
$$

and

$$
\cosh x=\frac{e^{x}+e^{-x}}{2}
$$

Hyperbolic functions may not be periodic functions as are the trigonometric functions; but many identities involving hyperbolic functions are quite similar to those for the corresponding trigonometric functions, as suggested by the names. 
The following identities remain valid and can be proved using definition 5.2 and properties of the exponential function. Proofs are left to the Check Your Understanding exercises.

\section{Property 1.15.1. Properties of the complex trigonometric functions}

If $z$ is complex numbers, then:

(a) $\sin (z+2 \pi)=\sin z$, and $\cos (z+2 \pi)=\cos z$

(b) $\sin (-z)=-\sin z$, and $\cos (-z)=\cos z$

(c) $\sin 2 z=2 \sin z \cos z$, and $\cos 2 z=\cos ^{2} z-\sin ^{2} z$

(d) $\sin ^{2} z+\cos ^{2} z=1$

Remark: Property 1.15.1 (a) tells us that $\sin z$ and $\cos z$ are both periodic with period $2 \pi$. This also tells us that $\sin z$ and $\cos z$ are not one-to-one functions, preventing them from have inverse functions.

\section{Worked Examples 1.15.1.}

Find complex number(s) $z$ that satisfy the following equations:

(a) $\cos z=2$

(b) $\sin z=2 i$

\section{Solutions to worked examples 1.15.1:}

(a) The method for these is always the same...we take the following steps:

(a) Write the trig function in terms of exponentials:

$$
\frac{e^{i z}+e^{-i z}}{2}=2
$$

(b) Multiply everything through by 2 in this case, and always by $e^{i z}$ :

$$
e^{2 i z}+1=4 e^{i z}
$$

(c) Let $w=e^{i z}$ so that we have a quadratic in $w$ :

$$
w^{2}-4 w+1=0
$$

(d) Complete the square:

$$
(w-2)^{2}-3=0
$$

(e) Solve the quadratic:

$$
w=2 \pm \sqrt{3}
$$

(f) Check that both of these solutions are positive (we have to do something slightly different if they're not.

(g) Now replace the $w$ with $e^{i z}$. Let $z=a+b i \Longrightarrow e^{i z}=e^{-b} e^{i a}$, and write the right hand side in complex exponential form:

$$
e^{-b} e^{i a}=2 \pm \sqrt{3} e^{2 \pi i k}, \quad k \in \mathbb{Z}
$$

(h) Match the modulus and argument of both sides:

$$
e^{-b}=2 \pm \sqrt{3} \Longrightarrow b=-\ln (2 \pm \sqrt{3}), a=2 \pi k
$$


(i) Write $z$ in terms of these $a$ and $b$ that you've just found:

$$
z=2 \pi k-i \ln (2 \pm \sqrt{3}), \quad k \in \mathbb{Z}
$$

(b) Very similar to the last, but we'll see a subtlety:

(a) Write the trig function in terms of exponentials:

$$
\frac{e^{i z}-e^{-i z}}{2 i}=2
$$

(b) Multiply everything through by $2 i$ in this case, and always by $e^{i z}$ :

$$
e^{2 i z}-1=4 i e^{i z}
$$

(c) Let $w=e^{i z}$ so that we have a quadratic in $w$ :

$$
w^{2}-4 i w-1=0
$$

(d) Complete the square:

$$
(w-2 i)^{2}+3=0
$$

(e) Solve the quadratic:

$$
w=2 i \pm \sqrt{3} i=i(2 \pm \sqrt{3})
$$

(f) Here are two complex roots. We have to write them in modulus argument form. Their modulus is $2 \pm \sqrt{3}$ and their argument is $\frac{\pi}{2}+2 \pi k, k \in \mathbb{Z}$. So again writing everything back in terms of $z=a+b i$ we have:

$$
e^{-b} e^{i a}=(2 \pm \sqrt{3}) e^{\frac{\pi}{2} i+2 \pi k i}
$$

Matching moduli and arguments we have

$$
z=\frac{\pi}{2}+2 \pi k-i \ln (2 \pm \sqrt{3})
$$

\section{Check your understanding 1.15.1.}

(a) Solve for $z \in \mathbb{C}$ if:

(i) $\sin z=4 i$

(ii) $\cos 2 z=2$

(iii) $\cos z=\sin z$

(iv) $\sin (i z)=\frac{e^{z}}{2}$

(b) Show that the formula $e^{i z}=\cos z+i \sin z$ holds true for all complex numbers $z$.

(c) Prove that $\sin z=0$ if, and only if, $z=k \pi$, where $k$ is an integer.

(d) Prove that $\cos z=0$ if, and only if, $z=\frac{\pi}{2}+k \pi$, where $k$ is an integer.

(e) Prove Properties 1.15.1.

\section{Section 2.15 Checklist}

$\square$ I know how $\sin z$ and $\cos z$ are related to $e^{i z}$ for a complex number $z$.

I can solve equation of the form $\sin z=w$ and $\cos z=w$ for a given complex number $w$.

$\square$ I know that lecturers are always willing to help, and I will consult them when I need clarifications. 


\subsection{Complex $n^{\text {th }}$ roots of a complex number}

If $i^{2}=-1$, does it mean that the square root of -1 is $i$, why not $-i$ ? Remember in section 1.2.3 we briefly discussed the freedom we have in Mathemafrica? The definition of the principle (real) square root (definition 1.2.1) required $\sqrt{x} \geq 0$ for $x \geq 0$, so there is no ambiguity when we take the principal square root of, for instance 9 , because $\sqrt{9}=+3$. Unfortunately, complex numbers cannot be ordered (see 1.8 for details) i.e. they are neither positive nor negative, so the square root of a complex number is ambiguous. We can't have the "positive" complex square root.

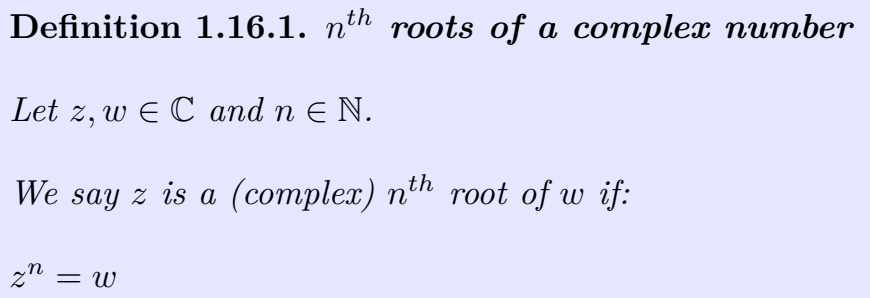

As an example you would say that \pm 1 are the complex $2^{\text {nd }}$ roots of 1 because $( \pm 1)^{2}=1$

Remark: A special case of this (when $w=1$ ) is solving $z^{n}=1$, the solutions to this equation are called roots of unity.

So you can ask questions like "what are the sixth roots of $i$ ?" which means, for which values of $z \in \mathbb{C}$ does $z^{6}=i$

Let's look at an example. Let's suppose we are interested in finding the cube roots $\left(3^{r d}\right)$ of $i$, what we are really looking for is all the complex numbers which when we cube we get $i$. If we put this into an equation, we are looking for all the values of $z \in \mathbb{C}$ that satisfy $z^{3}=i$. Let's solve for $z$ :

The first thing we will do EVERY TIME we solve one of these will be to write the complex number whose roots we are looking for in exponential form. In this case we need to put $i$ in the form $r e^{i \theta}$, where $r$ and $\theta$ are the modulus and argument of $i$. Well, $|i|=1$ and $\arg (i)=\frac{\pi}{2}+2 \pi k$ where $k \in \mathbb{Z}$, so:

$$
i=e^{i\left(\frac{\pi}{2}+2 \pi k\right)}
$$

The second thing we do is to write $z$ in exponential form, where this time of course we don't know the modulus and argument so we just write $z=r e^{i \theta}$ and this gives us:

$$
\left(r e^{i \theta}\right)^{3}=e^{i\left(\frac{\pi}{2}+2 \pi k\right)}
$$

which can be written as:

$$
r^{3} e^{3 i \theta}=e^{i\left(\frac{\pi}{2}+2 \pi k\right)}
$$

Now for the left and right hand sides to be equal (ie two complex numbers be equal to each other) their moduli and arguments have to be the same.

The modulus each side is $r^{3}$ on the left hand side and 1 on the right hand side, so we have $r^{3}=1$, but $r$ has to be real and positive, and there is only one such number for which this is true.... which is just $r=1$. For the arguments, we have $3 \theta$ on the left, and $\left(\frac{\pi}{2}+2 \pi k\right)$ on the right, so we must have:

$$
\theta=\frac{\pi}{6}=\frac{2}{3} \pi k
$$

where $k \in \mathbb{R}$

So the solutions are:

$$
z=1 e^{i\left(\frac{\pi}{6}=\frac{2}{3} \pi k\right)}
$$

We know that for a second root equation there are two solutions, but here it looks like we have an infinite number of solutions, one for each $k$...but actually this isn't quite true. Let's look at what these numbers are in Cartesian form for different $k$ 


$$
\begin{aligned}
& k=0 \Rightarrow z_{0}=e^{i \frac{\pi}{6}}=\frac{\sqrt{3}}{2}+\frac{1}{2} i \\
& k=1 \Rightarrow z_{1}=e^{i\left(\frac{\pi}{6}+\frac{2 \pi}{3}\right)}=\frac{-\sqrt{3}}{2}+\frac{1}{2} i \\
& k=2 \Rightarrow z_{2}=e^{i\left(\frac{\pi}{6}+\frac{4 \pi}{3}\right)}=-i \\
& k=3 \Rightarrow z_{2}=e^{i\left(\frac{\pi}{6}+\frac{6 \pi}{3}\right)}=e^{i\left(\frac{\pi}{6}+2 \pi\right)} e^{i \frac{\pi}{6}}=\frac{\sqrt{3}}{2}+\frac{1}{2} i
\end{aligned}
$$

See what happened there at $k=3$. The $k$ part ended up adding on $2 \pi$ to the argument, and you can take any argument and add on $2 \pi$ and you'll get back to the same place. It just says go around the circle a full turn.

Check yourself that for $k=4$ you will get back to the $k=1$ solution, and for $k=5$ you will get back to the $k=2$ solution. In fact $k=0,1,2$ give us all of the solutions.

We can plot the three solutions and they all lie on the unit circle:

See the visual representation of the three cube roots overleaf.

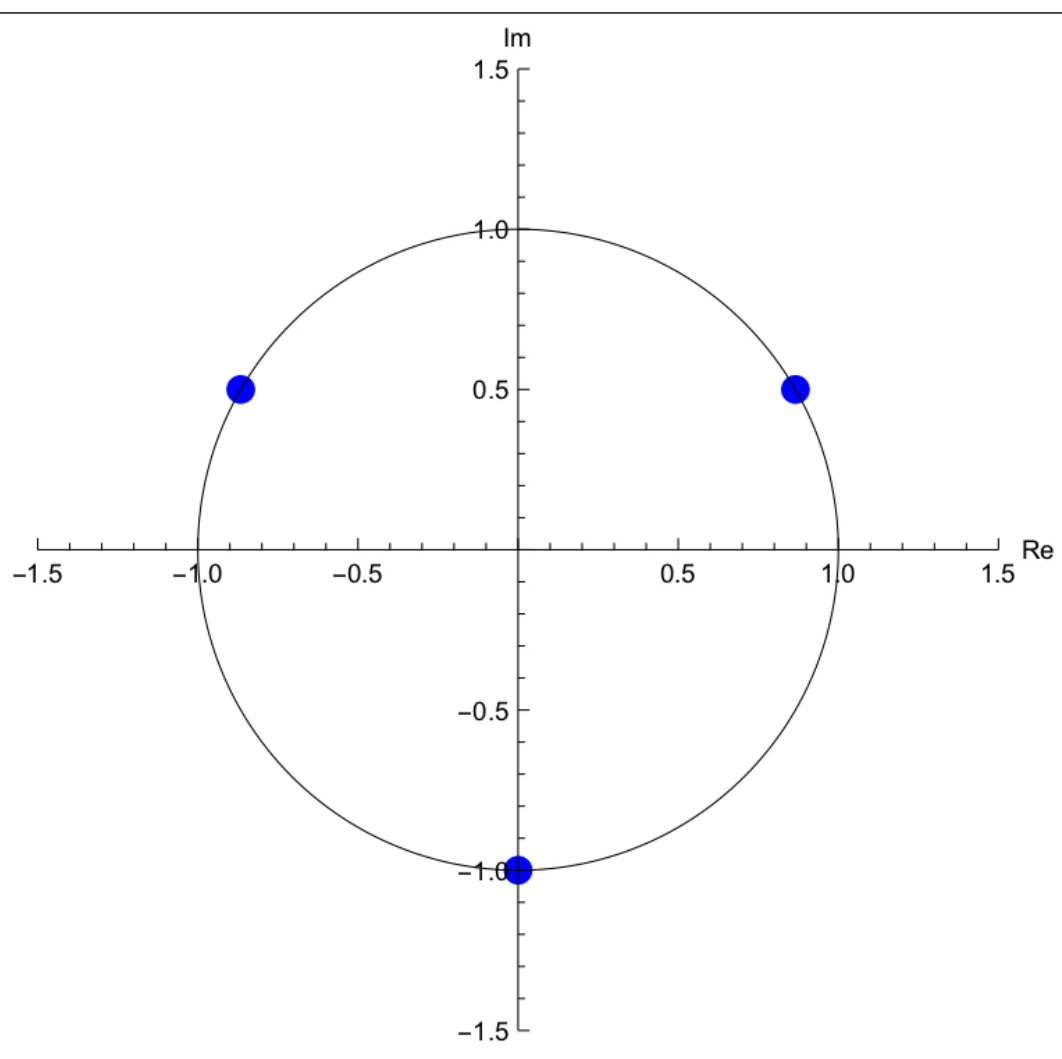

Figure 1.34: Three cube roots of $i$ sketched on an Argand diagram

Let's look at another example

Solve $z^{4}=1-i$

Again, we follow these steps:

1. Write left and right hand side in exponential form

2. Equate the modulus and argument of both sides

3. Solve for $r$ and $\theta$

4. Find the unique arguments (the values of $k$ for which the complex number is unique). This will always be $k=0$ to $k=n-1$ when you are trying to find the $n^{\text {th }}$ roots. 
5. write out the solutions in Cartesian form and plot them in the complex plane.

Let's see:

1. $1-i=\sqrt{2} e^{i\left(-\frac{\pi}{4}+2 \pi k\right)}, k \in \mathbb{Z}, \mathrm{so}:$

$$
r^{4} e^{4 i \theta}=\sqrt{2} e^{i\left(-\frac{\pi}{4}+2 \pi k\right)}
$$

2. Equating the modulus:

$$
r^{4}=\sqrt{2} \Longrightarrow r=2^{\frac{1}{8}}
$$

Equating the arguments:

$$
4 \theta=\left(-\frac{\pi}{4}+2 \pi k\right) \Longrightarrow \theta=\left(-\frac{\pi}{16}+\frac{\pi k}{2}\right)
$$

3.

$$
r=2^{\frac{1}{8}}
$$

and

$$
\theta=\left(-\frac{\pi}{16}+\frac{\pi k}{2}\right)
$$

4.

$$
\theta=\left\{-\frac{\pi}{16}, \frac{7 \pi}{16}, \frac{15 \pi}{16}, \frac{23 \pi}{16}\right\}
$$

5. Plugging these values of $\theta$ and $r$ into $r(\cos \theta+i \sin \theta)$ we get the solutions (up to four decimals):

$$
z=\{-1.0696+0.2127 i,-0.2127-1.0696 i, 0.2127+1.0696 i, 1.0696-0.2127 i\}
$$

which in the complex plane look like

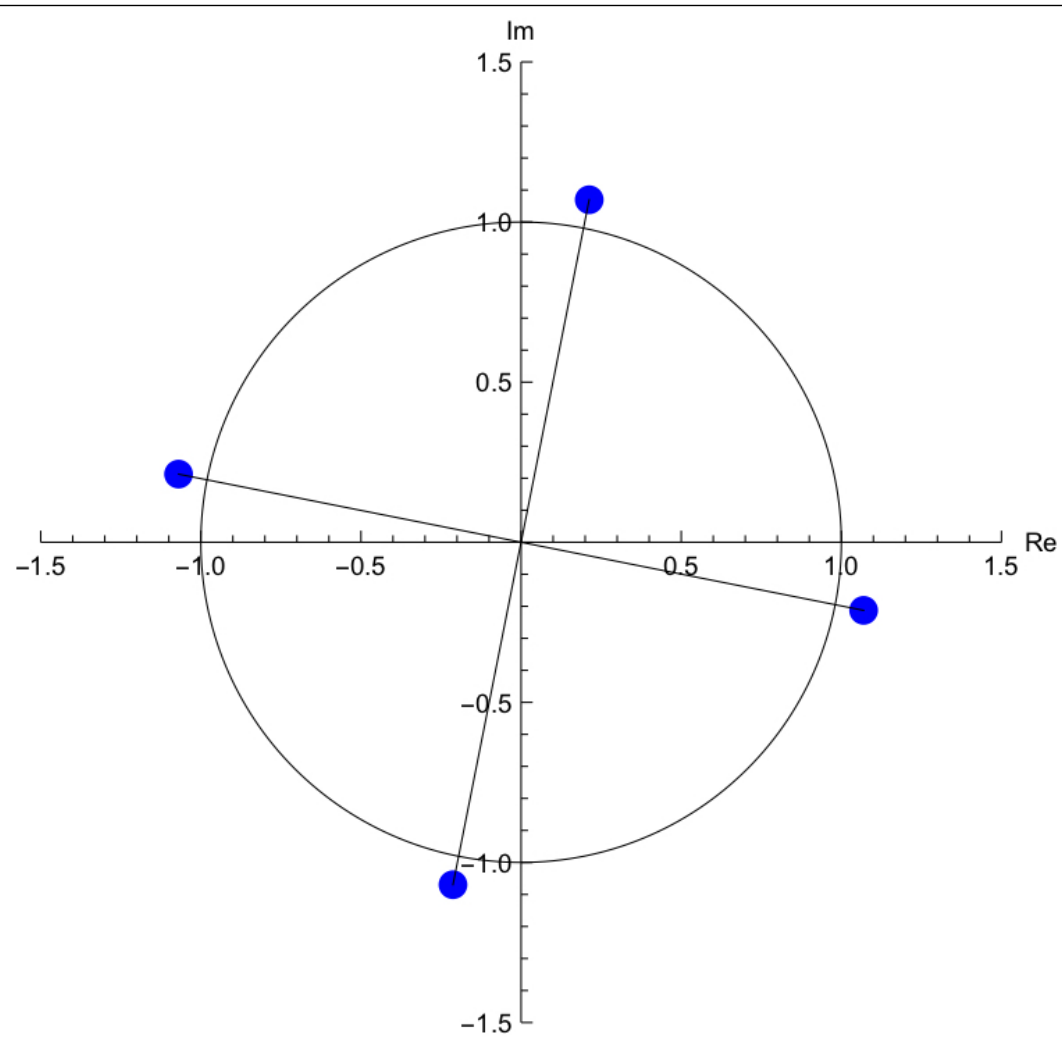

Figure 1.35: Four fourth roots of $1-i$ sketched in the complex plane along with the unit circle. The lines joining them to the origin have been put in to illustrate that the difference in arguments is $\frac{\pi}{2}$. 
It is a general rule that the $n n^{\text {th }}$ roots of a complex number will appear all with the same modulus and with equal spacing in terms of the argument. That spacing will always be $\frac{1}{n}$ of a circle.

\section{Worked Examples 1.16.1.}

(a) Find all the fourth roots of 16.

(b) Let $w \in \mathbb{C}$, and $n \in \mathbb{N}$. How many (complex) $n^{t h}$ roots does $w \neq 0$ have? This is a theorem!

(c) Find all the values for $z \in \mathbb{C}$ (and plot them on an Argand diagram) that satisfy the following equations:

(i) $z^{5}=1+i$

\section{Solutions to worked examples 1.16.1:}

(a) As before, we are trying to find $z$ such that:

$$
z^{4}=16
$$

Write both sides in complex exponential form:

$$
r^{4} e^{4 i \theta}=16 e^{2 \pi i k}, \quad k \in \mathbb{Z}
$$

Matching moduli and arguments we have:

$$
r^{4}=16 \Longrightarrow r=2,4 \theta=2 \pi k \Longrightarrow \theta=\frac{\pi k}{2}
$$

Thus:

$$
z=2\left(\cos \frac{\pi k}{2}+i \sin \frac{\pi k}{2}\right)=\{2,2 i,-2,-2 i\}
$$

We know that taking $k=0,1,2,3$ will give us all four solutions. We will plot the solution to this, and questions (c) after that question.

(b) There are always $n n^{\text {th }}$ roots of $w \neq 0$.

(c) As in the first part:

$$
z^{5}=r^{5} e^{5 i \theta}=1+i=\sqrt{2} e^{i \frac{\pi}{4}+2 \pi i k}
$$

Matching modulus and argument gives:

$$
r=\sqrt{2}^{\frac{1}{5}}=2^{\frac{1}{10}}, \quad \theta=\frac{\pi}{20}+\frac{2}{5} \pi k
$$

and we have to take $k=0,1,2,3,4$ :

$$
z=2^{\frac{1}{10}}\left(\cos \left(\frac{\pi}{20}+\frac{2}{5} \pi k\right)+i \sin \left(\frac{\pi}{20}+\frac{2}{5} \pi k\right)\right), k=0,1,2,3,4
$$

The solutions to this, as well as to part (a) are shown in the following figure: 


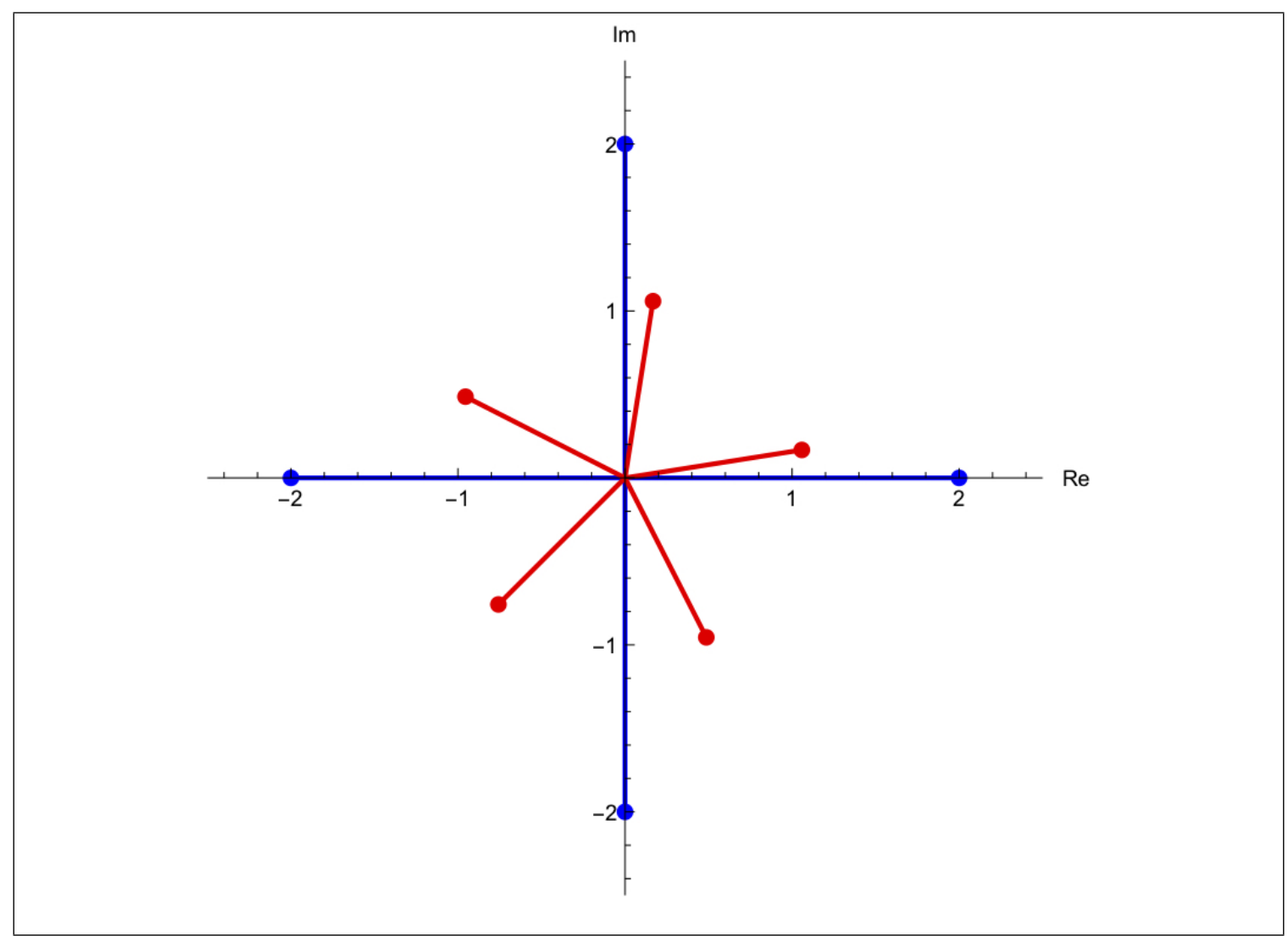

Figure 1.36: The fourth and fifth roots from (a) and (b) above.

\section{Check your understanding 1.16.1.}

(a) Find all the fourth roots of an imaginary number $-81 i$ and plot them on an Argand diagram.

(b) Find all the fifth roots of $\frac{1}{2}-i \frac{\sqrt{3}}{2}$

(c) Find all sixth roots of unity and plot them on an Argand diagram.

(d) Solve the following equations. List your solutions in modulus-argument form and plot them on an Argand diagram.

(i) $z^{5}=-1+\sqrt{3} i$

(ii) $z^{6}=-\sqrt{3}-i$

(iii) $z^{5}=32 i$

\section{Section 2.16 Checklist}

$\square$ I know what it means for a complex number $z$ to be an $n^{\text {th }}$ of another given complex number $w$.

$\square$ I can prove that a non-zero complex number has $n$ distinct $n^{\text {th }}$ roots.

$\square$ I know how to plot all the $n^{\text {th }}$ roots of a non-zero complex number on an Argand diagram.

$\square$ I know how to solve equations of the form $z^{n}=w$, where $w$ is a given complex number. 


\subsection{Zeros of polynomials in a complex variable}

We conclude our discussion of functions of a complex variable with one of our favorite functions, polynomials. Motivated by the definition of real polynomials of a real variable, we start by defining complex polynomials of a complex variable and their zeros. We will then use these definitions to explore interesting theorems about zeros of real polynomials of a complex variable.

Definition 1.17.1. Complex polynomials of a complex variable and their zeros

A complex polynomial in a complex variable $z$ is an expression of the form:

$$
p(z)=c_{0}+c_{1} z+c_{2} z^{2}+\ldots . .+c_{n-1} z^{n-1}+c_{n} z^{n}
$$

where the coefficients $c_{0}, c_{1}, c_{2}, \ldots ., c_{n}$ are complex numbers i.e. $c_{i} \in \mathbb{C}$ and $n \in \mathbb{N}$.

The degree of $p(z)$ is $n$ (if $c_{n} \neq 0$ ).

A complex number $\alpha$ is called a zero of $p(z)$ if $p(\alpha)=0$.

Remark: In some texts, you will see the terms roots and zeros used interchangeably. Strictly speaking, the term roots should be used for equations, and the term zeros should be used for functions.

Consider the real polynomial $p$ in a complex variable $z$ :

$$
p(z)=z^{2}+2 z+2
$$

Suppose we want to find the zeros of $p(z)$. We would proceed as follows:

$$
\begin{aligned}
& z^{2}+2 z+2=0 \\
& z^{2}+2 z+1+1=0 \\
& (z+1)^{2}+1=0 \\
& (z+1)^{2}=i^{2}
\end{aligned}
$$

It follows that $z+1=i$ or $z+1=-i$ solves the equation; the zeros of $p(z)$ are thus $-1+i$ and $-1-i$. We observe that the two roots are a complex conjugate pair, is this a coincidence?

\section{Theorem 1.17.1. Non-real zeros of a real polynomial}

The non-real zeros of a polynomial with real coefficients i.e.

$$
p(z)=a_{0}+a_{1} z+a_{2} z^{2}+\ldots . .+a_{n-1} z^{n-1}+a_{n} z^{n}
$$

(where $a_{n} \neq 0$ and the coefficients $a_{i} \in \mathbb{R}$ )

occur in complex conjugate pairs. That is, if $p(\alpha)=0$ then $p(\bar{\alpha})=0$.

Remark: A real polynomial is a polynomial with real coefficients. 
Study the proof of Theorem 1.17.1 below and answer the questions that follow:

\section{Proof of Theorem 1.17.1}

Let $\alpha$ be a non-real zero of a polynomial of degree $n$ with real coefficients i.e.

$$
p(\alpha)=a_{0}+a_{1} \alpha+a_{2} \alpha^{2}+\ldots .+a_{n-1} \alpha^{n-1}+a_{n} \alpha^{n}=0
$$

We shall now show that the conjugate of $\alpha$ is also a zero of $p(z)$ i.e. $p(\bar{\alpha})=0$.

$$
\begin{aligned}
p(\bar{\alpha}) & =a_{0}+a_{1} \bar{\alpha}+a_{2}(\bar{\alpha})^{2}+\ldots . .+a_{n-1}(\bar{\alpha})^{n-1}+a_{n}(\bar{\alpha})^{n} \\
& =a_{0}+a_{1} \bar{\alpha}+a_{2} \overline{\alpha^{2}}+\ldots . .+a_{n-1} \overline{\alpha^{n-1}}+a_{n} \overline{\alpha^{n}} \\
& =\overline{a_{0}}+\overline{a_{1}} \bar{\alpha}+\overline{a_{2}} \overline{\alpha^{2}}+\ldots . .+\overline{a_{n-1}} \overline{\alpha^{n-1}}+\overline{a_{n}} \overline{\alpha^{n}} \\
& =\overline{a_{0}}+\overline{a_{1} \alpha}+\overline{a_{2} \alpha^{2}}+\ldots . .+\overline{a_{n-1} \alpha^{n-1}}+\overline{a_{n} \alpha^{n}} \\
& =\overline{a_{0}+a_{1} \alpha+a_{2} \alpha^{2}+\ldots . .+a_{n-1} \alpha^{n-1}+a_{n} \alpha^{n}} \\
& =\overline{p(\alpha)} \\
& =\overline{0} \\
& =0
\end{aligned}
$$

\section{Questions (Please get your answers checked by your tutor/lecturer)}

(a) Where in the proof did we use the fact that the coefficients $a_{0}, a_{1}, a_{2} \ldots a_{n-1}, a_{n} \in \mathbb{R}$ ?

(b) What property of the complex conjugate makes the expressions at (1) and (2) equal?

(c) Explain why expressions in (2) and (3) are equal.

(d) What property of the complex conjugate makes expressions at (3) and (4) equal?

(e) What property of the complex conjugate makes the expressions at (4) and (5) equal?

(f) Explain why expressions in (5) and (6) are equal.

(g) Where in the proof do we use the fact that $\alpha$ is a zero of $p(z)$ ?

Generally if you are asked to write out this proof you will also have to be clear about why you were allowed to take each step using the properties of complex numbers that we've defined throughout this chapter.

For an example, suppose we are asked to find a polynomial of the form:

$$
p(z)=z^{2}+b z+c \quad b, c \in \mathbb{R}
$$

and are given that $1+i$ is one of the zeros of $p(z)$, how can we use Theorem 7 (a) to help us solve the problem?

Since $p(z)$ has real coefficients, we know that the conjugate of $1+i$ is also a zero of $p(z)$, so we have $p(1+i)=p(1-i)=0$ which means $z-(1+1)$ and $z-(1+i)$ are both factors of $p(z)$.

$$
\begin{aligned}
p(z) & =(z-(1+i))(z-(1-i)) \\
& =((z-1)-i)((z-1)+i) \\
& =(z-1)^{2}-i^{2} \\
& =z^{2}-2 z+1+1 \\
& =z^{2}-2 z+2
\end{aligned}
$$


Before we upgraded our number system from one dimension to two dimensions, we used to have polynomials that had no (real) zeros. Now that we allow non-real zeros, can a polynomial have no zeros at all? The Fundamental Theorem of Algebra guarantees us that a non-constant polynomial will always have at least one zero.

\section{Theorem 1.17.2. The Fundamental Theorem of Algebra}

Every non-constant polynomial with complex coefficients has at least one complex zero.

Remark: We will not show the proof of the FTA in this course, you'll probably see the proof in MAM3000W. It is believed that Gauss provided the first proof of this theorem in his doctoral dissertation in 1799. Although he only proved it for polynomials with real coefficients, it was not difficult to extend the theorem for polynomials with complex coefficients. Several proofs of the FTA have since been devised in several areas of mathematics including topology and complex analysis (read some proofs here). Some mathematicians actually think that the term "The Fundamental Theorem of Algebra" is inaccurate because the proposition is actually part of complex analysis, calling it "The Fundamental Theorem of Complex Numbers".

The FTA actually tells us something about the number of zeros of a polynomial of degree $n$.

\section{Corollary 1.17.3. Corollary of the fundamental theorem of algebra}

Any polynomial of degree $n \geq 1$ has $n$ zeros counted with multiplicity.

Remark: In some texts, you might see the FTA stated exactly the same way Corollary 7 is stated. Counting roots with multiplicity means that the polynomial $p(z)=z^{2}+2 z+1=$ $(z+1)(z+1)$ has two repeated zeros namely -1 and -1 , so when a zero is repeated $n$ times, we count it $n$ times. Corollary 7 can also be stated as "Any non-constant polynomial of degree $n$ has at most $n$ distinct zeros".

\section{Proof of Corollary 1.17.3:}

Let $P_{n}(z)$ be a polynomial of degree $n \geq 1$. By the Fundamental Theorem of Algebra (Theorem 1.17.2), $P_{n}(z)$ must have at least one zero, say $r_{1}$.

Since $r_{1}$ is a zero of $P_{n}(z)$, by the factor theorem, $\left(z-r_{1}\right)$ is a factor of $P_{n}(z)$. Implying that:

$P_{n}(z)=\left(z-r_{1}\right) Q(z) \quad$ where $Q(z)$ is a polynomial of degree $n-1$.

If $n-1>0$, then $Q(z)$ has at least one zero (by the FTA), say $r_{2}$.

Continuing in this fashion one may extract $n$ factors of $P(z)$

$P_{n}(z)=c\left(z-r_{1}\right)\left(z-r_{2}\right)\left(z-r_{3}\right) \cdots\left(z-r_{n-1}\right)\left(z-r_{n}\right)$ where $c$ is a constant to be determined.

Thus $P_{n}(z)$ has $n$ zeros counted with multiplicity.

Remark: It is important to bear in mind that the zeros $r_{i} \in \mathbb{C}$ are not necessarily distinct.

In the earlier sections of the semester when we used partial fraction decomposition to evaluate integrals, we assumed that we could factor every real polynomial into linear and/or irreducible quadratic factors, we are now ready to prove this! 
Theorem 1.17.4. Every non-constant real polynomial can be written as the product of real linear polynomial(s) and/or irreducible real quadratic polynomial(s).

\section{Proof of Theorem 1.17.4}

Consider any zero of a real polynomial $p(z)$ call it $R$.

If $R$ is real then $z-R$ is a real linear factor.

If $R$ is non-real then $\bar{R}$ is also a zero of $p(z)$ (Theorem 7(a)), then $(z-R)$ and $(z-\bar{R})$ are factors of $p(z)$.

So $(z-R)(z-\bar{R})$ is a factor.

$$
\begin{aligned}
(z-R)(z-\bar{R}) & =z^{2}-\bar{R} z-R z+R \bar{R} \\
& =z^{2}-(\bar{R}+R) z+R \bar{R} \\
& =z^{2}-2 \operatorname{Re}(R) z+|R|^{2}
\end{aligned}
$$

Properties 1.8.1(e) and 1.8.1(d)

$z^{2}-2 \operatorname{Re}(R) z+|R|^{2}$ is a real quadratic factor of $p(z)$

The discriminant of this quadratic is:

$$
\begin{array}{rrr}
\Delta & =(-2 \operatorname{Re}(R))^{2}-4(1)(|R|)^{2} & \\
& =4(\operatorname{Re}(R))^{2}-4|R|^{2} & \\
& =4(\operatorname{Re}(R))^{2}-4\left((\operatorname{Re}(R))^{2}+(\operatorname{Im}(R))^{2}\right) & \text { Definition 1.7.2 (a) } \\
& =-4(\operatorname{Im}(R))^{2} & \\
& <0 & \mathbf{R} \text { is not real, so } \operatorname{Im}(R) \neq 0
\end{array}
$$

So $z^{2}-2 \operatorname{Re}(R) z+|R|^{2}$ is an irreducible real quadratic factor.

This means that a polynomial of degree $n \geq 1$ with real coefficients can be factorized into real linear factors and/or real irreducible quadratic factors.

\section{Worked Examples 1.17.1.}

(a) Given that $p(2-i)=0$, write $p(z)=2 z^{3}-9 z^{2}+14 z-5$ as a product of a linear factor and a real irreducible quadratic factor. Find the real zero of $p(z)$.

(b) Consider the function $g(z)=z^{4}-2 z^{3}+2 z^{2}-10 z+25$. Given that $2+i$ is one of the zeros of $g(z)$, factorize $g$ completely.

\section{Solutions to worked examples 1.17.1}

(a) $p(z)$ is a polynomial with real coefficients, therefore all non-real roots come in conjugate pairs. Thus if $p(2-i)=0$, we must have that $(z-(2-i))$ and $(z-(2+i))$ are factors. Given this, then:

$$
(z-(2-i))(z-(2+i))=z^{2}-4 z+5
$$

is a factor. We then know that this must divide $p(z)$. Doing polyonomial long division we 
find:

$$
\frac{2 z^{3}-9 z^{2}+14 z-5}{5-4 z+z^{2}}=2 z-1
$$

Therefore $(2 z-1)=2\left(z-\frac{1}{2}\right)$ is a factor and so $\frac{1}{2}$ is the real root of this cubic.

(a) As in the previous example $(z-(2+i))(z-(2-i))=z^{2}-4 z+5$ must be a factor of $g$. Again, dividing $g(z)$ by this factor gives:

$$
\frac{z^{4}-2 z^{3}+2 z^{2}-10 z+25}{z^{2}-4 z+5}=z^{2}+2 z+5
$$

This quadratic is factored by completing the square to give two more roots: $z=-1-2 i$ and $z=-1+2 i$, thus the fully factored polynomial is

$$
g(z)=(z-(2+i))(z-(2-i))(z-(-1-2 i))(z-(-1+2 i))
$$

\section{Check your understanding 1.17.1.}

(a) Find all four zeros of $p(z)=z^{4}+1$ and use them to deduce the factorization $z^{4}+1=$ $\left(z^{2}-\sqrt{2} z+1\right)\left(z^{2}+\sqrt{2} z+1\right)$.

(b) The polynomial $p(z)=z^{4}-5 z^{3}+2 z^{2}+22 z-20$ has a zero at $z=3-i$. Factorize $p(z)$ as a product of real linear and real irreducible quadratic factors.

(c) Let $q(z)=z^{4}+1$ and $p(z)=z^{5}-3 z^{4}+z-3$.

(i) Find all the complex zeros of $q(z)$ and plot them on an Argand diagram.

(ii) Show that there is a polynomial $r(z)$ such that $p(z)=r(z) q(z)$ and hence find all the zeros of $p(z)$.

(iii) Factorize $p(z)$ completely into linear and real irreducible quadratic factors.

(d) Two of the solutions of a cubic equation (with real coefficients) in the complex variable $z$, are $1+2 i$ and 2 . If the constant term is equal to -30 , find the coefficient of $z^{3}$.

(e) Given that $3+i$ is a solution of the equation

$$
z^{4}-6 z^{3}+6 z^{2}+24 z-40=0
$$

find the other solutions of the equation.

(f) Given that $i$ is a zero of the polynomial $p(z)=z^{4}+4 z^{3}+14 z^{2}+4 z+13$, find all complex zeros of $p(z)$.

(g) The complex number $2+i$ is a zero of the polynomial $p(z)=3 z^{3}-14 z^{2}+23 z-10$. Use this information to factorize the real-valued polynomial $p(x)=3 x^{3}-14 x^{2}+23 x-10$ into real linear and irreducible quadratic factors.

\section{Section 2.17 Checklist}

$\square$ I know what a zero of a polynomial in a complex variable is.

I know that a real polynomial is a polynomial with real coefficients.

I can prove that non-real roots of a real polynomial occurs in complex conjugate pair.

$\square$ I know that any polynomial of degree $n \geq 1$ is guaranteed to have at least one root by The Fundamental Theorem of Algebra.

$\square$ I can prove that The Fundamental Theorem of Algebra implies that any polynomial of degree $n \geq 1$ has $n$ zeros counted with multiplicity. 
$\square$ I can prove that every non-constant real polynomial can be written as the product of real linear polynomials and/or irreducible real quadratic polynomials.

$\square$ I can factorize any real polynomial completely given at least one of its zeros.

$\square$ I will give constructive feedback on this pilot chapter. 